\title{
Properties and performance of the prototype instrument for the Pierre Auger Observatory
}

AUGER COLlaboration, J. Abraham ${ }^{\mathrm{a}}$, M. Aglietta ${ }^{\mathrm{b}}$, I.C. Aguirre ${ }^{\mathrm{c}}$, M. Albrow ${ }^{\mathrm{d}}$, D. Allard ${ }^{\mathrm{e}}$, I. Allekotte ${ }^{\mathrm{f}}$, P. Allison ${ }^{\mathrm{g}}$, J. Alvarez Muñiz ${ }^{\mathrm{bb}}$, M.G. do Amaral ${ }^{i}$, M. Ambrosio ${ }^{\mathrm{j}}$, L. Anchordoqui ${ }^{\mathrm{k}}$, R. Andrews ${ }^{\mathrm{d}}$, M. Anguiano ${ }^{1}$, J.C. dos Anjos ${ }^{\mathrm{m}}$, C. Aramo ${ }^{\mathrm{j}}, \mathrm{S}$. Argiro ${ }^{\mathrm{n}}$, K. Arisaka ${ }^{\mathrm{O}}$, J.C. Arteaga ${ }^{\mathrm{p}}$,

S. Atulugama ${ }^{\mathrm{g}}$, M. Ave ${ }^{\mathrm{q}}$, G. Avila ${ }^{\mathrm{r}}$, R. Baggio ${ }^{\mathrm{n}}$, X. Bai ${ }^{\mathrm{bj}}$, A.F. Barbosa ${ }^{\mathrm{m}}$, H.M.J. Barbosas ${ }^{\mathrm{s}}$, D. Barnhillo, S.L.C. Barroso ${ }^{\mathrm{s}}$, P. Bauleo ${ }^{\mathrm{t}}$, J. Beatty ${ }^{\mathrm{g}}$, T. Beau ${ }^{\mathrm{u}}$, K.-H. Becker ${ }^{\mathrm{v}}$, J.A. Bellido ${ }^{\mathrm{w}}$, P. Bello ${ }^{1}$, T. Bergmann ${ }^{\mathrm{x}}$, E. Berman ${ }^{\mathrm{d}}$, X. Bertou ${ }^{\mathrm{y}}$, P. Biermann ${ }^{\text {bk }}$, P. Billoir ${ }^{\mathrm{z}}$, R. Biral ${ }^{\mathrm{s}}$, H. Bluemer ${ }^{\mathrm{x}}$, M. Bohacova ${ }^{\mathrm{aa}}$, E. Bollmann ${ }^{\mathrm{x}}$, C. Bonifazi ${ }^{\mathrm{ab}}$, M. Boratav ${ }^{z}$, A. Boselli ${ }^{\mathrm{ab}}$, J. Brack $^{\mathrm{ac}}$, J.-M. Brunet $^{\mathrm{u}}$, H. Bui-Duc ${ }^{\mathrm{ap}}$, V. Cabreral, D.V. Camin ${ }^{\text {ae }}$, J.-N. Capdevielle ${ }^{\mathrm{u}}$, A. Carreño ${ }^{\text {af }}$, N. Cartiglia ${ }^{\mathrm{n}}$, R. Caruso ${ }^{\text {ag }}$, L.A. de Carvalho ${ }^{\mathrm{s}}$, S. Casanova ${ }^{\text {bk }}$, E. Casimiro ${ }^{\mathrm{p}}$, A. Castellina ${ }^{\mathrm{b}}$, J. Castro ${ }^{1}$, P.W. Cattaneo ${ }^{\text {ah }}$, L. Cazon ${ }^{\text {bb }}$, R. Cester ${ }^{\text {n }}$, N. Chávez ${ }^{\text {ai }}$, D. Cheam ${ }^{\text {aj }}$, A. Chiavassa $^{\text {b }}$, J.A. Chinellatos, M. Chiosson ${ }^{\text {n }}$ A. Chou ${ }^{\mathrm{d}}$, J. Chye $^{\mathrm{aj}}$, A. Cillis $^{\mathrm{a}}$,

B. Civit ${ }^{\mathrm{a}}$, D. Claes ${ }^{\mathrm{a}}$, P.D.J. Clark ${ }^{\mathrm{am}}$, R.W. Clay ${ }^{\mathrm{w}}$, F. Cohen ${ }^{\mathrm{u}}$, A. Cordero, A. Cordier ${ }^{\text {an }}$, E. Cormier ${ }^{\text {an }}$, J. Cotzomi $^{1}$, U. Cotti ${ }^{\text {ai }}$, S. Coutu ${ }^{\text {g }}$, C.E. Covault ${ }^{\text {ao }}$,

A. Creusot ${ }^{\mathrm{e}}$, J.W. Cronin ${ }^{\mathrm{y}}$, M. Cuautle, S. Dagoret-Campagne ${ }^{\mathrm{z}}$,

T. Dang-Quang ${ }^{\text {ap }}$, P. Da Silva ${ }^{z}$, J. Darling ${ }^{\text {aj }}$, P. Darriulat ${ }^{\text {ap }}$, K. Daumiller ${ }^{\text {aq }}$, B.R. Dawson ${ }^{\mathrm{w}}$, L. de Bruijn ${ }^{\mathrm{am}}$, A. De Capoa ${ }^{\mathrm{n}}$, M.A.L. de Oliveira ${ }^{\mathrm{s}}$, V. de Souza ${ }^{\mathrm{s}}$, A. Della Selva ${ }^{\mathrm{j}}$, O. Deligny ${ }^{\mathrm{z}}$, J.C. Diaz ${ }^{\mathrm{aj}}$, C. Dobrigkeit ${ }^{\mathrm{s}}$, J.C. D’Olivo $^{\mathrm{af}}$, A. Dorofeev ${ }^{a j}$, M.T. Dova ${ }^{\text {ak }}$, A. Dye ${ }^{\text {am }}$, M.A. DuVernois ${ }^{\text {ar }}$, R. Engel ${ }^{\mathrm{x}}$, L.N. Epele ${ }^{\mathrm{ak}}$, P. Eschstruth ${ }^{\mathrm{an}}$, C.O. Escobar ${ }^{\mathrm{s}}$, A. Etchegoyen $^{\mathrm{ab}}$,

P. Facal San Luis ${ }^{\text {as }}$, A.C. Fauth ${ }^{\mathrm{s}}$, N. Fazzinir ${ }^{\mathrm{r}}$, A. Fernández, A.M.J. Ferrero ${ }^{\mathrm{ab}}$, B. Fick ${ }^{\mathrm{y}}$, A. Filevich ${ }^{\mathrm{ab}}$, A. Filipčič ${ }^{\mathrm{at}}$, R. Fonte ${ }^{\mathrm{aw}, \mathrm{bc}}$, W. Fulgione ${ }^{\mathrm{b}}$, E. Gámez, B. Garcia ${ }^{\mathrm{a}}$, C.A. Garcia ${ }^{\mathrm{f}}$, H. Geenen ${ }^{\mathrm{v}}$, H. Gemmeke ${ }^{\text {av }}$, C. Germain-Renaud ${ }^{\text {an }}$, P.L. Ghia ${ }^{\text {b }}$, K. Gibbs ${ }^{y}$, M. Giller ${ }^{\mathrm{h}}$, J. Gitto ${ }^{\mathrm{a}}$, H. Glass ${ }^{\mathrm{d}}$, M. Gómez Berisso ${ }^{\mathrm{f}}$, P.F. Gomez Vitale ${ }^{\mathrm{r}}$, J. González ${ }^{\text {, }}$, J. González ${ }^{\mathrm{k}}$, D. Gora ${ }^{\text {ax }}$, A. Goodwin ${ }^{\mathrm{ay}}$, P. Gouffon ${ }^{\mathrm{az}}$, V. Grassi ${ }^{\text {ae }}$, A.F. Grillo ${ }^{\text {ba }}$,

\footnotetext{
*Corresponding author. Department of Physics and Astronomy, University of Leeds, Leeds LS2 9JT, UK. Tel.: +44-113-34333888; fax: + 44-113-3433900

E-mail address: a.a.watson@leeds.ac.uk (A.A. Watson).
} 
C. Grunfeld ${ }^{\mathrm{ak}}$, J. Grygar ${ }^{\mathrm{aa}}$, F. Guarino ${ }^{\mathrm{j}}$, G. Guedes $^{\mathrm{m}}$, C. Guerard ${ }^{\mathrm{aq}}$, R. Gumbsheimer ${ }^{\mathrm{x}}$, J.L. Harton ${ }^{\mathrm{t}}$, F. Hasenbalg ${ }^{\mathrm{ab}}$, D. Heck ${ }^{\mathrm{x}}$, J.M. Hernández, D. Hoffer ${ }^{\mathrm{d}}$, C. Hojvat ${ }^{\mathrm{d}}$, P. Homola ${ }^{\text {ax }}$, M. Horvat ${ }^{\text {at }}$, M. Hrabovsky ${ }^{\text {aa }}$, A. Insolia ${ }^{\text {au }}$, S. Jaminion ${ }^{g}$, Y. Jerónimo ${ }^{\text {ai }}$, L. Jiang ${ }^{\text {bj }}$, M. Kaducak ${ }^{\mathrm{d}}$, K.-H. Kampert ${ }^{\mathrm{v}}$, B. Keilhauer ${ }^{\mathrm{x}}$, E. Kemp ${ }^{\mathrm{s}}$, H. Klages ${ }^{\mathrm{x}}$, M. Kleifges ${ }^{\text {av }}$, J. Kleinfeller ${ }^{\mathrm{x}}$, J. Knapp ${ }^{\mathrm{q}}$, A. Kopmann $^{\text {av }}$, N. Kunka ${ }^{\text {av }}$, M. Kutschera ${ }^{\text {ax }}$, C. Lachaud ${ }^{\mathrm{u}}$, M. Lapolla ${ }^{\text {ae }}$, A. Letessier-Selvon ${ }^{z}$, I. Lhenry-Yvon ${ }^{\mathrm{e}}$, J. Lloyd-Evans ${ }^{\mathrm{q}}$, R. López, A. Lopez Aguera ${ }^{\text {bb }}$, M. Lucano ${ }^{\mathrm{m}}$, R. Luna ${ }^{\mathrm{p}}, \mathrm{Y}_{\text {. Ma }}{ }^{\mathrm{bj}}$, M.E. Manceñido ${ }^{\mathrm{ak}}$, P.F. Manfredi ${ }^{\text {ah }}$, L. Manhaes ${ }^{\mathrm{m}}$, P. Mantsch ${ }^{\mathrm{d}}$, A.G. Mariazzi ${ }^{\mathrm{ak}}$, M.J. Markus ${ }^{\mathrm{bc}}$,

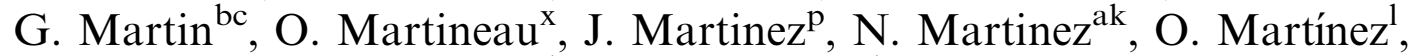
H.-J. Mathes ${ }^{\mathrm{x}}$, J.A.J. Matthews ${ }^{\text {bc }}$, J. Matthews ${ }^{\text {ad,ay }}$, G. Matthiae ${ }^{\text {as }}$, E. Marques ${ }^{\text {h }}$, P. Matussek ${ }^{\mathrm{x}}$, G. Maurin ${ }^{\mathrm{u}}$, D. Maurizion ${ }^{\mathrm{n}}$, P. Mazur ${ }^{\mathrm{d}}$, T. McCauley ${ }^{\mathrm{k}}$, M. McEwen ${ }^{\text {ay }}$, R.R. McNeil ${ }^{\text {ay }}$, C. Medina ${ }^{\text {ai }}$, M.C. Medina ${ }^{\text {ab }}$, G. Medina-Tanco ${ }^{\text {bd }}$, D. Melo ${ }^{\mathrm{ab}}$, M. Melocchi ${ }^{\mathrm{a}}$, E. Menichetti ${ }^{\mathrm{n}}$, A. Menshikov ${ }^{\mathrm{av}}$, F. Meyer ${ }^{\mathrm{be}}$, R. Meyhandan ${ }^{\text {ay }}$, J.C. Meza ${ }^{\mathrm{r}}$, G. Miele ${ }^{\mathrm{j}}$, W. Miller ${ }^{\mathrm{bc}}$, M. Mohammed ${ }^{\mathrm{aj}}$, D. Monnier-Ragaigne $^{\text {an }}$, C. Morello ${ }^{\text {, }}$, E. Moreno ${ }^{1}$, M. Mostafa ${ }^{n}$, R. Mussa ${ }^{n}$, H. Nassini ${ }^{f}$, G. Navarra ${ }^{\text {, }}$ L. Nellen ${ }^{\text {af }}$, F. Nerling ${ }^{\mathrm{x}}$, C. Newman-Holmes ${ }^{\mathrm{d}}$,

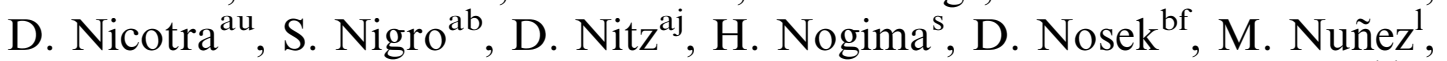
T. Ohnuki ${ }^{\mathrm{o}}$, A. Olinto ${ }^{\mathrm{y}}$, S. Ostaptchenko ${ }^{\mathrm{aq}}$, M. Palatka ${ }^{\mathrm{aa}}$, G. Parente ${ }^{\mathrm{bb}}$, E. Parizot ${ }^{\mathrm{e}}$, E.H. Pasaye ${ }^{\mathrm{ai}}$, N. Pastrone ${ }^{\mathrm{n}}$, M. Patel $^{\mathrm{q}}$, T. Paul $^{\mathrm{k}}$, I. Pedraza ${ }^{1}$, J. Pekala ${ }^{\mathrm{ax}}$, R. Pelayo ${ }^{\mathrm{p}}$, I.M. Pepe ${ }^{\mathrm{bg}}$, A. Pérez-Lorenzana ${ }^{\mathrm{p}}$, L. Perrone ${ }^{\mathrm{v}}$, N. Peshman ${ }^{\text {ar }}$, S. Petrera ${ }^{\text {ag }}$, P. Petrinca ${ }^{\text {as }}$, D. Pham-Ngoc ${ }^{\text {ap }}$, P. Pham-Trung ${ }^{\text {ap }}$, T. Pierog ${ }^{\mathrm{x}}$, O. Pisanti ${ }^{\mathrm{j}}$, N. Playez ${ }^{\text {an }}$, E. Ponce ${ }^{1}$, T.A. Porter ${ }^{\text {ay }}$, L. Prado Junior ${ }^{\mathrm{s}}$, P. Privitera ${ }^{\mathrm{as}}$, M. Prouza ${ }^{\mathrm{a} a}$, C.L. Pryke ${ }^{\mathrm{y}}$, J.B. Rafert ${ }^{\text {aj }}$, G. Raia ${ }^{\text {au }}$, S. Ranchon ${ }^{\text {an }}$, L. Ratti ${ }^{\text {ah }}$, D. Ravignani ${ }^{\text {ab }}$, V. Re ${ }^{\text {ah }}$, H.C. Reis ${ }^{\mathrm{s}}$, S. Reucroft ${ }^{\mathrm{k}}$, B. Revenue ${ }^{\mathrm{u}}$, M. Richter ${ }^{\mathrm{v}}$, J. Ridky $^{\mathrm{aa}}$, A. Risi $^{\mathrm{aw}}$, M. Risse ${ }^{\mathrm{x}}$, V. Rizi ${ }^{\mathrm{ag}}$, M.D. Roberts ${ }^{\mathrm{bc}}$, C. Robledo ${ }^{1}$, G. Rodriguez ${ }^{\mathrm{bb}}$, J. Rodriquez ${ }^{\text {aw }}$, J. Rodriquez Martino ${ }^{\text {ab }}$, S. Román ${ }^{1}$, L. Rosa $^{j}$, M. Roth ${ }^{\mathrm{x}, \mathrm{q}}$, A.C. Rovero ${ }^{\text {bh }}$, H. Salazar ${ }^{1}$, G. Salina ${ }^{\text {as }}$, F. Sanchez ${ }^{\text {ae }}$, M. Santander ${ }^{\text {aw }}$, L.G. dos Santos ${ }^{\text {s, R. Sato }}{ }^{\text {, P. Schovanek }}{ }^{\text {aa }}$, V. Scherini ${ }^{\text {ae }}$, S.J. Sciutto ${ }^{\text {ak }}$, G. Sequeiros-Haddad ${ }^{\text {ah }}$, R.C. Shellard ${ }^{\mathrm{m}}$, E. Shibuya ${ }^{\mathrm{s}}$, F.V. Siguas ${ }^{\mathrm{m}}$, W. Slater ${ }^{\mathrm{o}}$, N. Smetniansky-De Grande ${ }^{\mathrm{ab}}$, K. Smith ${ }^{\mathrm{t}}$, G.R. Snow ${ }^{\mathrm{al}}$, P. Sommers ${ }^{\mathrm{bi}}$, C. Song ${ }^{\text {ar }}$, H. Spinka ${ }^{d}$, F. Suarez ${ }^{\text {a }}$ T. Suomijärvi ${ }^{e}$, D. Supanitsky ${ }^{\text {ab }}$, J. Swain ${ }^{\mathrm{k}}$, Z. Szadkowski ${ }^{\mathrm{h}}$, A. Tamashiro ${ }^{\mathrm{ab}}$, G.J. Thornton ${ }^{\mathrm{w}}$, T. Thouw ${ }^{\mathrm{x}}$,

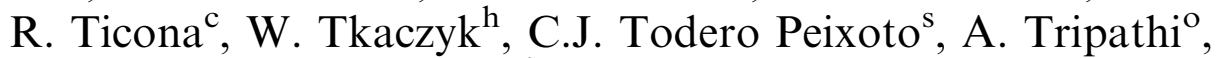
G. Tristram ${ }^{\mathrm{u}}$, M. Trombley ${ }^{\mathrm{aj}}$, D. Tscherniakhovski ${ }^{\mathrm{av}}$, P. Tuckey ${ }^{\text {be }}$, V. Tunnicliffe ${ }^{\mathrm{am}}$, M. Urban ${ }^{\text {an }}$, C. Uribe Estrada ${ }^{\text {an }}$, J.F. Valdés ${ }^{\text {af }}$,

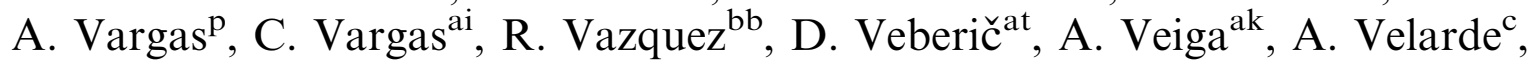


F. Vernotte ${ }^{\text {be }}$, V. Verzias ${ }^{\text {as }}$ M. Videla ${ }^{\mathrm{a}}$, C. Vigorito $^{\mathrm{b}}$, L.M. Villaseñor ${ }^{\mathrm{ai}}$, M. Vlcek ${ }^{\text {aa }}$, L. Voyvodic ${ }^{d}$, T. Vo-Van ${ }^{\text {ap }}$, T. Waldenmaier ${ }^{\mathrm{x}}$, P. Walker ${ }^{\text {am }}$, D. Warner ${ }^{\mathrm{t}}$, A.A. Watson ${ }^{\mathrm{q}, *}$, Ch. Wiebusch ${ }^{\mathrm{v}}$, G. Wieczorek ${ }^{\mathrm{h}}$, B. Wilczynska ${ }^{\text {ax }}$, H. Wilczynski ${ }^{\text {ax }}$, N.R. Wild ${ }^{\mathrm{w}}$, T. Yamamoto ${ }^{\mathrm{y}}$, E. Zas ${ }^{\mathrm{bb}}$, D. Zavrtanik ${ }^{\text {at }}$, M. Zavrtanik ${ }^{\text {at }}$, A. Zepeda ${ }^{\mathrm{p}}$, C. Zhang ${ }^{\text {bj }}$, Q. Zhu ${ }^{\text {bj }}$

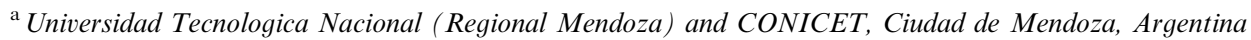 \\ ${ }^{\mathrm{b}}$ Istituto di Fisica dello Spazio Interplanetario, sezione di Torino and Dipartimento di Fisica Generale dell'Universitá and INFN Torino, \\ Via P. Giuria 1, 10125 Torino, Italy \\ ${ }^{\mathrm{c}}$ National Academy for Sciencies, Av. 16 Julio 1732, POB 5829, La Paz, Bolivia \\ ${ }^{\mathrm{d}}$ Fermilab, MS105, POB 500, Batavia, IL 60510-0500, USA \\ ${ }^{\mathrm{e}}$ Institut de Physique Nucléaire, Université Paris-Sud and IN2P3/CNRS, 91406 Orsay, France \\ ${ }^{\mathrm{f}}$ Instituto Balseiro, Centro Atomico Bariloche/CNEA y Universidad Nacional de Cuyo, (8400) San Carlos de Bariloche, \\ Rio Negro, Argentina \\ ${ }^{\mathrm{g}}$ Department of Physics, Pennsylvania State University, 104 Davey Laboratory, University Park, PA 16802, USA \\ ${ }^{\mathrm{h}}$ University of Lodz, Division of Experimental Physics, ul. Pomorska 149/153, 90-236 Lodz, Poland \\ ${ }^{\mathrm{i}}$ Universidade Federal Fluminense, Campus da Praia Vermelha, 24210-340 Niterói, RJ, Brazil \\ ${ }^{\mathrm{j}}$ Dipartimento di Fisica and INFN, Via Cintia 2, 80123 Napoli, Italy \\ ${ }^{\mathrm{k}}$ Department of Physics, Northeastern University, 110 Forsyth Street, Boston, MA 02115-5096, USA \\ ${ }^{1}$ Benemérita Universidad Autónoma de Puebla (BUAP), Ap. Postal J-48, 72500 Puebla, Puebla, Mexico \\ ${ }^{\mathrm{m}}$ Centro Brasileiro de Pesquisas Físicas, Rua Dr. Xavier Sigaud 150 22290-180 Rio de Janeiro, RJ, Brazil \\ ${ }^{\mathrm{n}}$ Dipartimento di Fisica Sperimentale and INFN Torino, Via P. Giurial, 10125 Torino, Italy \\ ${ }^{\circ}$ Department of Physics and Astronomy, University of California, Los Angeles (UCLA), Los Angeles, CA 90095, USA \\ ${ }^{\mathrm{p}}$ Centro de Investigacion y de Estudios Avanzados del IPN (CINVESTAV), Physics Department, POB 14-740, \\ 07000 Mexico D.F., Mexico \\ ${ }^{\mathrm{q}}$ Department of Physics and Astronomy, University of Leeds, Leeds, LS2 9JT, UK \\ ${ }^{\mathrm{r}}$ Pierre Auger Southern Observatory, Av. San Martin Norte 304 (5613) Malargüe, Prov. de Mendoza, Argentina \\ ${ }^{\mathrm{s}}$ Universidade Estadual de Campinas, Instituto de Física, Departamento de Raios Cósmicos e Cronologia, CP 6165, 13084-971, \\ Campinas SP, Brazil \\ ${ }^{\mathrm{t}}$ Department of Physics, Colorado State University, Fort Collins, CO 80523, USA \\ ${ }^{u}$ Laboratoire de Physique Corpusculaire et Cosmologie, Collège de France and IN2P3/CNRS and Université Paris 7, 75005 Paris, France \\ ${ }^{v}$ Universität Wuppertal, Fachbereich Physik, Gaußstr.20, D-42119, Wuppertal, Germany \\ ${ }^{\mathrm{w}}$ Department of Physics and Mathematical Physics, University of Adelaide, South Australia 5005, Australia \\ ${ }^{\mathrm{x}}$ Forschungszentrum Karlsruhe GmbH, Institut für Kernphysik (FZK-IK), 76021 Karlsruhe, Germany \\ ${ }^{\mathrm{y}}$ Enrico Fermi Institute, University of Chicago, $5640 \mathrm{~S}$. Ellis, Chicago, IL 60637, USA \\ ${ }^{\mathrm{z}}$ Laboratoire de Physique Nucléaire et de Hautes Energies, Université Paris 6 \& 7 and IN2P3/CNRS, 75005 Paris, France \\ ${ }^{\text {aa }}$ Institute of Physics, Czech Academy of Sciences, Na Slovance 2, CZ-18221 Praha 8, Czech Republic \\ ${ }^{\mathrm{ab}}$ Laboratorio Tandar, Comisión Nacional de Energía Atómica and CONICET, Av. Gral. Paz 1499 (1650) San Martin, \\ Buenos Aires, Argentina \\ ${ }^{\mathrm{ac}}$ Physics Department, University of Colorado, Boulder, CO 80309-0446, USA

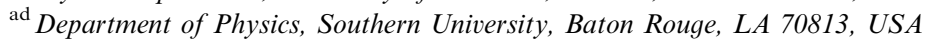

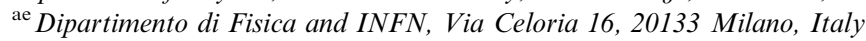 \\ ${ }^{\text {af }}$ Universidad Nacional Autónoma de México (UNAM), Apdo. Postal 70-542, 04510 Mexico D.F., Mexico \\ ${ }^{\mathrm{ag}}$ Dipartimento di Fisica and INFN, L'Aquila, Italy \\ ${ }^{a h}$ Universitá di Pavia, Universitá di Bergamo and INFN Pavia, Via Bassi 6, 27100 Pavia, Italy \\ ${ }^{\text {ai } U n i v e r s i d a d ~ M i c h o a c a n a ~ d e ~ S a n ~ N i c o l a s ~ d e ~ H i d a l g o ~(U M S N H), ~ A p d o . ~ P o s t a l ~ 2-82, ~} 58040$ Morelia, Michoacan, Mexico

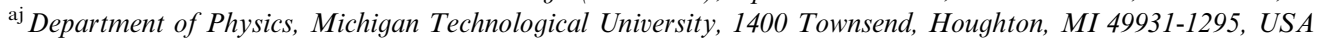 \\ ${ }^{\mathrm{ak}}$ Universidad Nacional de la Plata, Facultad de Ciencias Exactas, Departamento de Física and IFLP/CONICET, C.C. 67, \\ (1900) La Plata, Argentina \\ ${ }^{\mathrm{al}}$ Department of Physics and Astronomy, University of Nebraska, 116 Brace Lab, Lincoln, NE 68588-0111, USA \\ ${ }^{\mathrm{am}}$ Institute of Integrated Information Systems, School of Electronic Engineering, University of Leeds, Leeds, LS2 9JT, UK

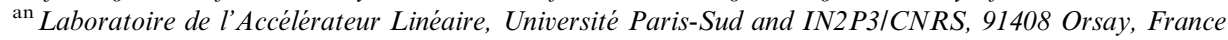

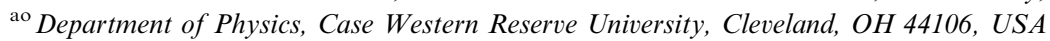 \\ ${ }^{\mathrm{ap}}$ Institute of Nuclear Science and Technology (INST), 5T-160 Hoang Quoc Viet Street, Nghia Do, Cau Giay, Hanoi, Viet Nam \\ ${ }^{\mathrm{aq}}$ Universität Karlsruhe (TH), Institut für Experimentelle Kernphysik (IEKP), 76128 Karlsruhe, Germany
}


${ }^{\mathrm{ar}}$ School of Physics and Astronomy, University of Minnesota, 116 Church St. SE, Minneapolis, MN 55455, USA

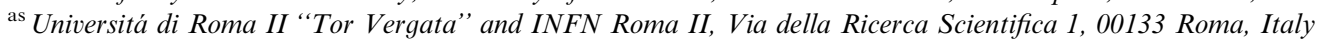

${ }^{a t}$ Nova Gorica Polytechnic, Laboratory for Astroparticle Physics, Vipavska 13, POB 301, 5001 Nova Gorica, Slovenia

${ }^{\text {au }}$ Dipartimento di Fisica dell'Universitá and INFN, Corso Italia 57, 95129 Catania, Italy

${ }^{a v}$ Forschungszentrum Karlsruhe GmbH, Institut für Prozessdatenverarbeitung und Elektronik (FZK-IPE), 76021 Karlsruhe, Germany

${ }^{\text {aw }}$ Universidad Tecnologica Nacional (Regional San Rafael), Ciudad de San Rafael, Argentina

${ }^{a x}$ Institute of Nuclear Physics, ul. Radzikowskiego 152, 31-342 Krakow, Poland

${ }^{a y}$ Department of Physics and Astronomy, Louisiana State University, Baton Rouge, LA 70803-4001, USA

${ }^{\text {az } U n i v e r s i d a d e ~ S a ̃ o ~ P a u l o, ~ I n s t i t u t o ~ d e ~ F i ́ s i c a, D e p a r t a m e n t o ~ d e ~ F i s i c a ~ E x p e r i m e n t a l, ~ C P ~ 66318, ~ 05315-970, ~ S a ̃ o ~ P a u l o, ~ S P, ~ B r a z i l ~}$

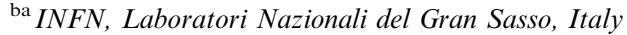

${ }^{\mathrm{bb}}$ Departamento de Fisica de Particulas, Universidad de Santiago de Compostela, Spain

${ }^{\mathrm{bc}}$ Physics Department, University of New Mexico, Albuquerque, NM 87131, USA

${ }^{b d}$ Instituto Astronômico e Geofisico, Universidade São Paulo, Rua do Matao 1226, 05508-900, São Paulo, SP, Brazil

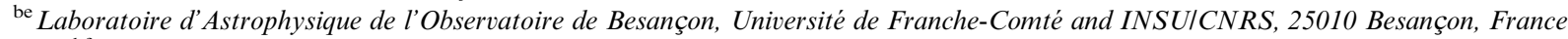

${ }^{\mathrm{bf}}$ Institute of Nuclear and Particle Physics, Faculty of Mathematics and Physics, Charles University, Praha, Czech Republic

bg Universidade Federal da Bahia, Campus da Ondina, 40210-340 Salvador, BA, Brazil

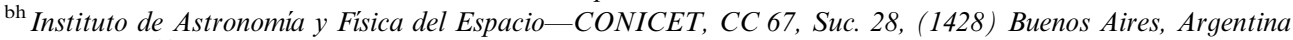

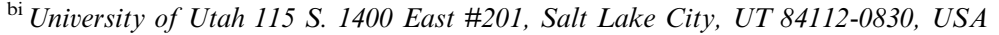

${ }^{b j}$ Institute of High Energy Physics, Chinese Academy of Sciences, Beijing 100039, People's Republic of China

${ }^{\mathrm{bk}}$ Max Planck Institut für Radioastronomie, 53121 Bonn, Germany

Received 13 November 2003; accepted 4 December 2003

\begin{abstract}
Construction of the first stage of the Pierre Auger Observatory has begun. The aim of the Observatory is to collect unprecedented information about cosmic rays above $10^{18} \mathrm{eV}$. The first phase of the project, the construction and operation of a prototype system, known as the engineering array, has now been completed. It has allowed all of the sub-systems that will be used in the full instrument to be tested under field conditions. In this paper, the properties and performance of these sub-systems are described and their success illustrated with descriptions of some of the events recorded thus far.
\end{abstract}

(C) 2003 Elsevier B.V. All rights reserved.

PACS: 95.85.Ry; 96.40.Pq

Keywords: Ultra high energy cosmic rays; Auger Observatory; Extensive air showers

\section{Introduction}

The Pierre Auger Observatory has been conceived to measure the flux, arrival direction distribution and mass composition of cosmic rays from $10^{18} \mathrm{eV}$ to the very highest energies with high statistical significance over the whole sky. To achieve this coverage, the Observatory will have instruments located at two sites, one in each of the Northern and Southern Hemispheres. The astrophysical interest in this energy range is well known, stemming largely from the expectation of spectral features in the decade above $10^{19} \mathrm{eV}$. In particular, it has been predicted $[1,2]$ that the energy spectrum should steepen sharply above about $6 \times 10^{19} \mathrm{eV}$ because of the interaction of primary cosmic rays with the microwave background radiation. There is considerable controversy $[3,4]$ about the existence, or not, of the predicted steepening, commonly known as the Greisen-Zatsepin-Kuzmin (GZK) cut-off. It is clear, however, that there are cosmic rays with energies well beyond $10^{20} \mathrm{eV}$ and major issues are the flux of these events and the accurate measurement of the spectral shape. It is known that the spectrum of cosmic rays extends to at least $3 \times 10^{20} \mathrm{eV}$. Recent reviews are available $[5,6]$.

Above $10^{20} \mathrm{eV}$, the rate of events is about $1 \mathrm{~km}^{-2} \mathrm{sr}^{-1}$ century ${ }^{-1}$, so that vast areas must be monitored to collect a large statistical sample. The 
Pierre Auger Observatory has been planned as a pair of arrays, each of $3000 \mathrm{~km}^{2}$. The design for the Southern Observatory calls for 1600 waterCherenkov detectors, arranged on a triangular grid, with the sides of the triangles being $1.5 \mathrm{~km}$, overlooked from four sites by optical stations, each containing six telescopes, designed to detect air-fluorescence light. The water tanks respond to the particle component (mainly muons, electrons and positrons and photons at the distances of importance) and the fluorescence cameras measure the emission from atmospheric nitrogen, which is excited by the charged particles of the shower as they traverse the atmosphere. Both techniques, already used for many years to study extensive air showers [7,8], are brought together in a 'hybrid' detector to observe showers simultaneously with different techniques. The array of water tanks is known as the surface detector (SD), while the optical stations form the fluorescence detector (FD).

The surface array will have the following properties:

- $100 \%$ duty cycle.

- A well-defined aperture that is independent of energy above $10^{19} \mathrm{eV}$.

- Uniform coverage in right ascension on a daily basis.

- A response that is largely independent of weather conditions.

- The quality of the data for each event improves with energy.

- Sensitivity to showers arriving at large zenith angles.

- In situ calibration of the detectors by cosmic ray muons.

- Measurement of the time structure of the arriving signals, which is sensitive to the mass of the primary particles.

The fluorescence detectors can be operated during clear nights with little moonlight and have the following characteristics:

- Every event above $10^{19} \mathrm{eV}$ is registered by at least one fluorescence detector: $60 \%$ of these events will be recorded by two or more fluorescence detectors. Essentially, every trans-
GZK event will be a stereo event. Multiple station coverage improves the energy resolution.

- A coincidence of a single detector of the surface array with a single fluorescence telescope constrains the shower geometry as precisely as a stereo fluorescence detector.

- The longitudinal development profile is measured directly.

- The fluorescence detectors provide a more direct measure of the shower energy. The small, unseen, fraction of the total energy carried by neutrinos and muons depends somewhat on the mass of the primary particle as well as on the hadronic interaction model.

The design for the Observatory was developed through a series of workshops, starting in Paris in 1992, and culminating in a 6-month study at Fermi National Accelerator Laboratory in 1995. The design is well suited to resolve the discrepancies at the high energy end of the cosmic ray spectrum that have been reported by the AGASA surface array [3] and the HiRes fluorescence detector [4].

Presently, the Southern Hemisphere Observatory of the planned pair is being built in Argentina. The first phase of the project, the construction and operation of a prototype system, known as the engineering array, has now been completed. This has allowed all of the sub-systems that will be used in the full instrument to be tested under field conditions. The Engineering Array comprises 32 fully instrumented water tanks and two FD telescopes at one site. In the following we describe the properties and performance of the sub-systems and illustrate their success with a description of some of the events recorded thus far.

Preliminary details concerning some of the material discussed here were presented at the 2001 International Cosmic Ray Conference in Hamburg and in the Design Report [9].

\section{Site of the Southern Observatory}

The goal of constructing a hybrid instrument led to the specification of the site characteristics. 
These include the need for location at an altitude between 500 and $1500 \mathrm{~m}$ above sea level (asl). In addition, the communications and deployment requirements make a relatively flat site desirable while the need to detect the faint fluorescence signals requires a location with optical characteristics close to those sought by astronomical telescopes. After evaluating several sites in Argentina, Australia and South Africa, a site in Argentina was selected for the southern part of the Observatory [10].

\subsection{Exposure and latitudes}

Broad sky coverage at each site is highly desirable in that it allows the whole sky to be observed with two detectors, provided appropriate latitudes are chosen. This factor was one of the important considerations in the selection of waterCherenkov detectors for the surface detector array. Water-Cherenkov detectors are relatively deep (e.g. $1.2 \mathrm{~m}$ at Haverah Park [7]), while scintillators commonly used in arrays are much thinner (e.g. $9 \mathrm{~cm}$ at Volcano Ranch [11]). A comparison of the declination distributions of showers recorded by scintillators and large water-Cherenkov EAS arrays at Volcano Ranch and Haverah Park emphasises this point. The FWHM of the declination distributions were $\approx 40^{\circ}$ and $\approx 75^{\circ}$, respectively, and as such, a water-Cherenkov array will have approximately twice as much sky coverage as would a scintillator array. A simulation, which reproduced the Haverah Park declination distribution, showed that two observatories at latitudes close to $\pm 35^{\circ}$ would give nearly uniform exposure of the sky with water-Cherenkov tanks [9].

\subsection{Pampa Amarilla site}

The Pampa Amarilla site $\left(35.1^{\circ}-35.5^{\circ} \mathrm{S}, 69.0^{\circ}-\right.$ $69.6^{\circ} \mathrm{W}$ and $1300-1400 \mathrm{~m}$ asl) lies in the south of the Province of Mendoza, Argentina, close to Malargüe (pop. 18000) and $180 \mathrm{~km}$ south west of San Rafael (pop. 100000 ). It encompasses an area of $3100 \mathrm{~km}^{2}$ (see Fig. 1a).

Before proceeding to the construction of the full-size observatory, a subset of it, the Engineering Array (EA) (Fig. 1b), has been built and operated. It consists of two fluorescence telescopes, located at Cerro Los Leones (see Fig. 1b) and 40 surface detector units deployed within the fields of view of the two telescopes. For the $\mathrm{SD}$, the construction and operation of the EA has allowed the testing of tanks (materials, liners, solar panels, brackets, cabling, etc.), water production and quality, photomultiplier (PMT) tubes, electronics, triggers, software and data acquisition, monitoring packages and telecommunications, as well as of the deployment strategy. For the FD, it has been possible to evaluate the performance of the aperture stop, corrector ring, UV-pass filter, mirror, camera, phototubes, analogue and digital electronics, data acquisition, shutter and slow control.

The centre of the SD Engineering Array lies $10 \mathrm{~km}$ north of the FD building at Los Leones. Of the 40 tanks deployed, 32 were completely instrumented. Of the detectors, 37 were positioned on a triangular grid, covering an area of approximately $46 \mathrm{~km}^{2}$. The mean deviation of these tanks from a perfect grid is $21 \mathrm{~m}$, with a maximum deviation of $90 \mathrm{~m}$ for one of the stations. Near the centre of this array, a further detector was placed $11 \mathrm{~m}$ from an existing one. This pair of detectors enables comparisons of timing and density measurements to be made at essentially the same distance from the shower core. Additionally, two detectors were deployed at the middle of two of the triangles of the detector pair, i.e. at $860 \mathrm{~m}$ from their three nearest neighbours, allowing triggering on lower energy showers.

A large proportion of the infrastructure for the complete observatory is already in place. Assembly and office buildings (approximately $800 \mathrm{~m}^{2}$ each), buildings for the fluorescence detectors at Los Leones and Coihueco (each over $300 \mathrm{~m}^{2}$ ), three telecommunication towers, and the power lines to the buildings have been commissioned. The two FD buildings are, respectively, 40 and $150 \mathrm{~m}$ above the area of the SD. The data acquisition system is operative and data are being recorded in a stable manner.

Legally binding contracts have been signed with the majority of landowners, by which the collaboration gains access to the detectors over a period of 20 years. 

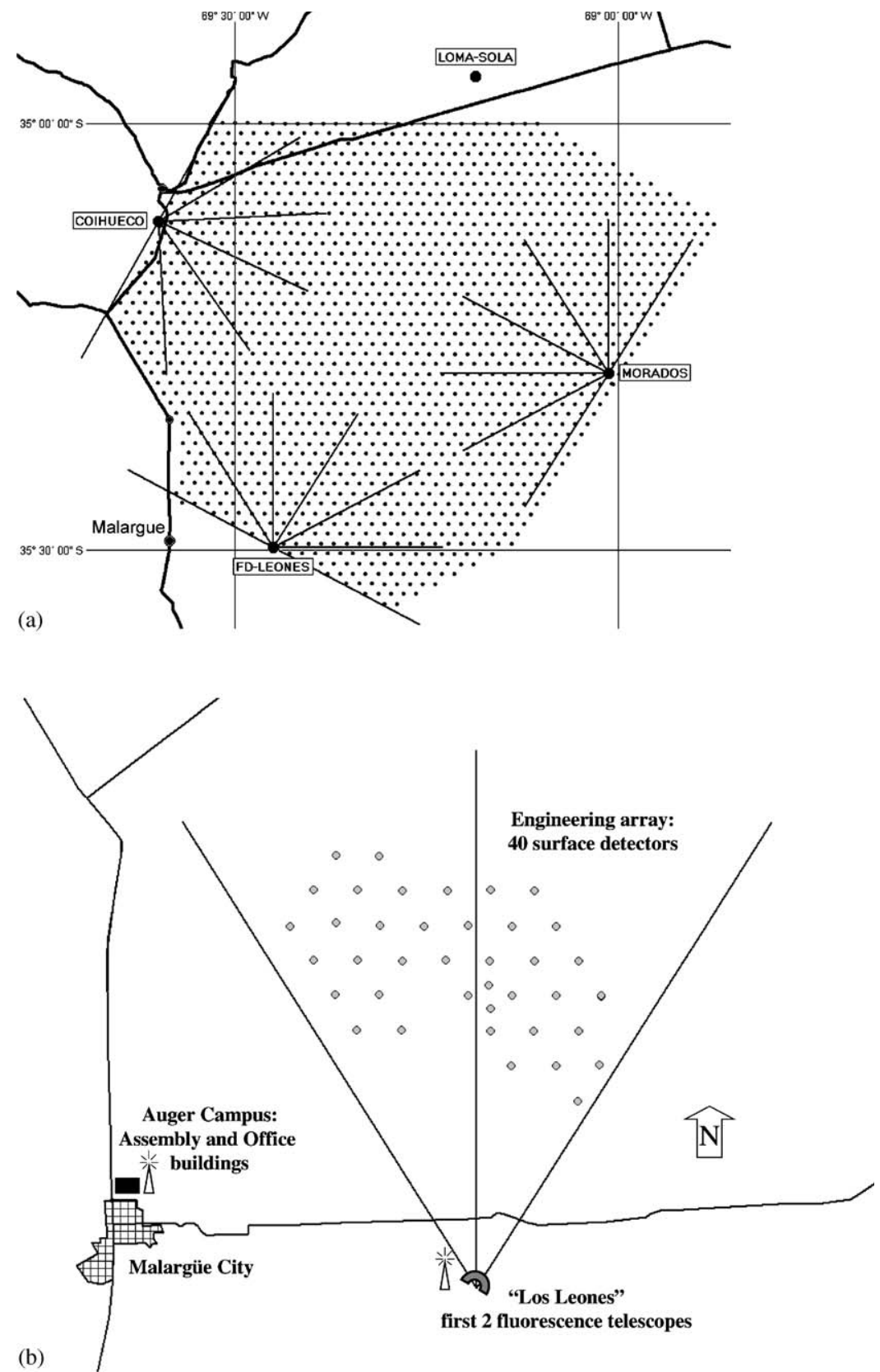

Fig. 1. (a) Layout of the Observatory, where 1600 surface detectors will be deployed within the dotted area. The four fluorescence telescope sites are shown at Cerro Los Leones, Coihueco, Los Morados, and at the northern periphery of the array. The lines encompass $30^{\circ}$ angles and define the azimuth acceptance of each fluorescence telescope. (b) The layout of the Engineering Array: 32 tanks were fully instrumented. The distance between neighbouring detectors is usually $1.5 \mathrm{~km}$. 


\subsection{Fluorescence detectors and atmospheric transparency}

The SD system has a potential duty cycle of $100 \%$, while the fluorescence detector system has a duty cycle limited to about $10 \%$, as data can only be taken on clear nights with little moonlight. The layout of the Observatory has been arranged so that events registered by the telescopes will nearly always be 'hybrid', with additional data coming from one or more of the water tanks of the $\mathrm{SD}$. The properties of the site required for the FD telescopes are a long attenuation length in the atmosphere at the appropriate wavelengths $(300-400 \mathrm{~nm})$ and many nights per year with clear skies.

There is little man-made air or light pollution at the site as the only place with population nearby, Malargüe, is a small city without major industries. Several studies of the atmosphere at Pampa Amarilla were carried out. Air quality measurements, including an estimation of fog occurrence and determinations of aerosol contents and attenuation lengths, were performed. The results indicate that most of the fog occurs during the day or at twilight. If the phase of the Moon is included in the analysis, then the overlap of FD potential runtime and fog is $85 \mathrm{~h}$ in 13 lunar months, which is to be compared to the expected $900 \mathrm{~h} \mathrm{year}^{-1}$ of fluorescence detector operation.

The first FD station, at which the data described below were recorded, was sited at Los Leones, on a small ridge to the south of the SD array and $40 \mathrm{~m}$ above the Pampa at an elevation of $1390 \mathrm{~m}$ asl. A second station building has been constructed to the west of the array at Coihueco on a mountain ridge at an elevation of $1650 \mathrm{~m}$ asl. A third station is being built at Los Morados to the east at $1400 \mathrm{~m}$ asl with the fourth station planned for Loma Sola to the north at $1420 \mathrm{~m}$ asl.

Three sets of measurements have been made that relate to the question of atmospheric transparency.

Measurements of the vertical transmission were made using a telescope set to follow a few standard bright stars and measure their luminosity at several zenith angles. The telescope was fitted with a Hamamatsu PMT with a U-Johnson filter centred at $365 \mathrm{~nm}$. Measurements were made on 22 nights between November 1997 and August 1998. For vertical transmission in the atmosphere, it is convenient to describe the extinction in terms of the number of vertical air masses that must be traversed for the light beam to be attenuated by $1 / e$. The average attenuation length obtained was 2.06 air masses. Seasonal modulation was observed, with lower opacity during the summer months. A value of 1.88 air masses, obtained during the preliminary site survey in autumn 1996, is consistent with these later results. By comparison, in a perfectly clear 'Rayleigh' atmosphere, the corresponding figure for $365 \mathrm{~nm}$ and a height of $1.5 \mathrm{~km}$ is 2.2 air masses.

A second set of measurements was made in 1998 to obtain the horizontal attenuation length in the B-band, centred at $550 \mathrm{~nm}$. The method was based on the comparison of light intensities, measured with a CCD camera, emitted by two distant lamp sources 14.79 and $43.94 \mathrm{~km}$ from the camera. The horizontal attenuation lengths were found to be in the range $17-37 \mathrm{~km}$. Measurements on 36 nights between 7 July and 13 November 1998 gave a mean of $31 \mathrm{~km}$, for a filter centred on $440 \mathrm{~nm}$ with a $380-520 \mathrm{~nm}$ range. By comparison, the corresponding Rayleigh attenuation length in this band is $39 \mathrm{~km}$.

More recently, the clarity of the atmosphere was monitored routinely during the period of hybrid data taking. Measurements of the total horizontal atmospheric attenuation at a wavelength of $365 \mathrm{~nm}$ were made using the prototype of a horizontal attenuation monitor (HAM). The HAM system ran automatically, providing clarity data three times nightly for the final 3 months of the hybrid run. The mean total horizontal attenuation length at $365 \mathrm{~nm}$ for this data set was $13.3 \mathrm{~km}$ with an rms spread of $2.5 \mathrm{~km}$. In all, $90 \%$ of the hybrid measurements were made on nights with a total horizontal attenuation length of greater than $9.5 \mathrm{~km}$. Note that with no aerosols, the total horizontal attenuation length, set by Rayleigh scattering, is about $18.7 \mathrm{~km}$.

The results from these three sets of measurements on atmospheric transparency indicate that the atmosphere is clear at the site of the Southern Auger Observatory. 


\subsection{Tank deployment}

The water tanks give the possibility of broad coverage in declination. However, they are relatively difficult to deploy over a large area with few roads. Deployment (and maintenance to a lesser extent) dictated the need for a site with relatively flat features and with low and scarce vegetation, availability of dirt tracks and few landowners.

The chosen site is suitable in as much as it is a "pampa" (i.e. a flat extensive area) with several gravel and dirt roads. However, $\sim 30 \%$ of the area is soft and wet during winter and rainy periods, and here vehicles are prone to sink under weight. During the EA phase, substantial experience has been gained with water delivery.

\section{Surface detectors of the engineering array}

A photograph and a schematic diagram of an EA surface detector unit are shown in Fig. 2a and $b$, respectively. Each Cherenkov detector consists of a rotationally moulded polyethylene tank, $3.6 \mathrm{~m}$ diameter and $1.55 \mathrm{~m}$ high, enclosing a liner filled with 120001 of high purity water. During the period of EA operation, combinations of 8 and 9 in. diameter PMTs were used with two types of viewing windows. The water in each tank is observed through three hard (polycarbonate) or soft (clear polyethylene) windows set into the top surface of the liner. Signals from the PMTs are read by the electronics mounted locally at the station. Power is provided by batteries connected to two solar panels, and time synchronisation relies on a commercial GPS receiver using a technique described in Ref. [12]. A specially designed radio system is used to provide communication between the surface detectors and the central computers of the Observatory (see Section 5). The Yagi antenna, used for communications, and the solar panel array are shown in Fig. 2c. Each tank forms an autonomous unit, recording signals from the ambient cosmic ray flux, independent of the signals registered by any other tanks in the SD array. A combination of signals clustered in space and time is used to identify a shower (see Section 6 for details).

\subsection{Polyethylene tank}

The tank structure encloses a cylindrical volume of water $1.2 \mathrm{~m}$ deep and of $10 \mathrm{~m}^{2}$ area. The top of the tank has been designed to house three photomultipliers, as well as to provide some rigidity. It has to support the stresses brought by the solar panels in strong winds and, occasionally, the weight of up to three people working on top of it. In addition, it must be resistant to long-term creep distortion due to its own weight. Three hatches allow access to each of the PMTs. One hatch, $560 \mathrm{~mm}$ in diameter, is used to connect the PMTs to the electronics enclosure. The other two hatches are $450 \mathrm{~mm}$ in diameter and have no electrical connections passing through them.

This design has been implemented by building rotationally moulded tanks from the polyethylene resin Escorene HD-8661 supplied by Exxon-Mobil Chemical, Canada. It has a density of $0.942 \mathrm{~g} \mathrm{~cm}^{-3}$, a melt index of $2.0 \mathrm{~g} / 10 \mathrm{~min}$ and the reputation of producing long-life products with good moulding properties, a necessity to assure a smooth interior.

To guarantee the opacity of the tank, this resin is hot-compounded with $1 \%$ of carbon black pigment. However, to reduce the ecological impact of black tanks in the sandy and yellowish landscape of Pampa Amarilla, and the effect of heating during sunny days, the tank is rotomoulded in two layers, the external one being compounded with a beige pigment. The internal black layer is about twice as thick as the external one, giving a total wall thickness of around $13 \mathrm{~mm}$. Resistance to UV degradation is insured by using a Hindered Amine Light Stabiliser (HALS) in the resin.

\subsection{Tank liner}

The liner is a cylindrical Polyolefin bag used to contain the detector volume inside the tank. It is mechanically supported by the tank, but must provide a 20 year seal for the water, high reflectivity of Cherenkov light and act as a secondary seal against extraneous light sources. Additionally, it must protect the water from contamination and inhibit bacteriological activities. The liner is made of a three layer lamination of polyolefin plastics: there 


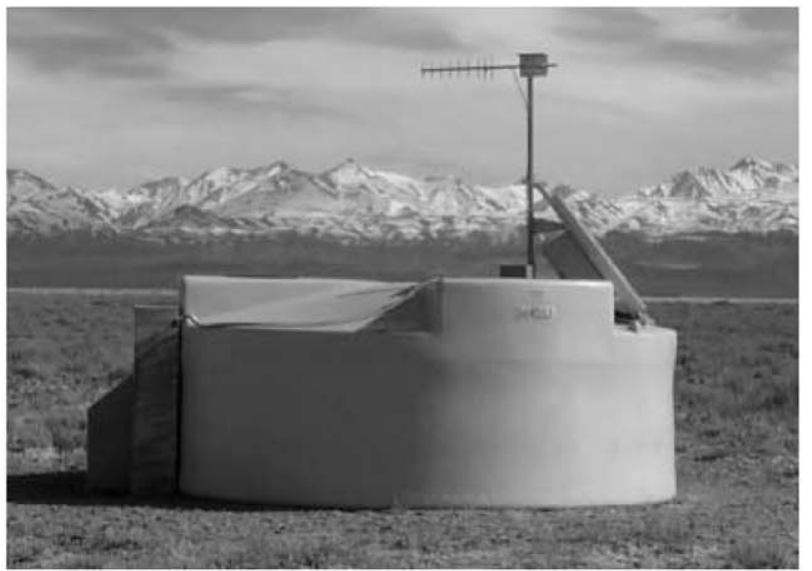

(a)
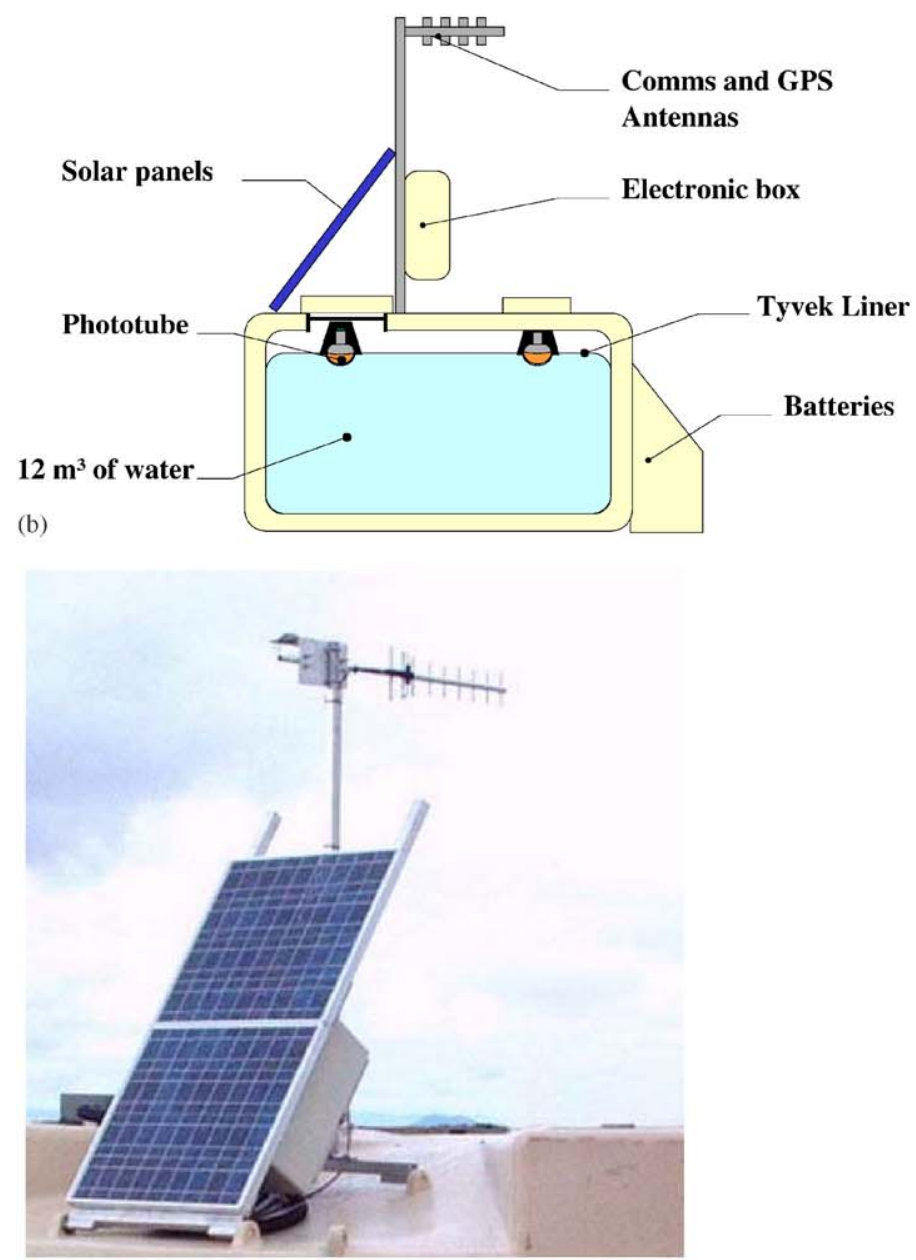

(c)

Fig. 2. (a) A photograph of an EA water tank; (b) schematic view of an EA tank; (c) the Yagi antenna and the solar power array. 
is an inner $140 \mu \mathrm{m}$ thick sheet of DuPont Tyvek ${ }^{\circledR}$ BL-25, giving excellent diffuse reflectivity of Cherenkov light and mechanical strength to the material, a $28 \mu \mathrm{m}$ thick Dow 722 LDPE (low-density polyethylene) film and a $178 \mu \mathrm{m}$ black polyethylene film specially designed to provide a water and light barrier, while preventing the carbon black pigment from contaminating the detector volume.

At a distance of $1.2 \mathrm{~m}$ from the centre of the top of the tank, at three symmetric locations in the top of the liner, polyethylene dome windows are fitted for the installation of the PMTs. The caps through which the water filling is done are also part of the assembly. The dome window units are made up of a custom-molded polyethylene flange (Dow $722 \mathrm{M}$ hot-compounded with $10 \%$ carbon black by weight) heat sealed to a flexible, transparent LDPE film dome (Dow 1880). The dome is custom-formed to match the PMT front face. The dome/flange unit is then heat sealed to the liner lamination to provide an hermetic seal for the liner. The PMT is optically coupled to the dome window using an optical coupling compound (GE RTV 6196 D1). With the coupling compound, the dome window transmits approximately $90 \%$ of the light seen by a PMT face directly in the water. The PMT and the associated electronics are enclosed in a custommolded plastic cover, which is sealed using custom molded clips and a silicone RTV gasket to the molded flange to protect the PMT from both ambient light and environmental effects.

In addition to the flexible windows, which have been chosen for the use in the full array, a rigid, UV-transmitting acrylic dome was tested in the engineering array. The rigid domes were formed to fit the various PMT faces, and transmitted fractionally more light than the LDPE domes. While the rigid domes could provide more protection to the optical seal between the PMT and the dome in the event of freezing of the water, these domes require an adhesive to bond to the flange. The heat-seal between the flexible window and the flange was found to be easier to make, more reliable, and does not introduce a new substance to be in contact with the water. In addition, the freezing concern has been solved by using two insulating rings, one inside the PMT cover and one outside the cover.

\subsection{Water production for the tanks}

The tanks are filled with ultra-pure water so that its properties will be maintained over the lifetime of the experiment, envisaged as 20 years. For this purpose, a water purification plant has been installed at the Malargüe Observatory, producing water at a rate that allows the filling of around three tanks per day. The water goes through different steps of filtering, softening, de-ionisation by reverse osmosis, destruction of organic carbon by $185 \mathrm{~nm}$ and bacteria by $254 \mathrm{~nm}$ UV light, and electro-deionisation. The water produced is stored in a 500001 storage tank, located near the water plant, with a recirculation system that includes $254 \mathrm{~nm}$ UV irradiation to maintain low biological activity and a mixed resin bed to maintain high water resistivity.

Some 12 tons of ultra-pure water must be delivered to each of the 1600 detectors at remote locations and as each detector requires 120001 of water, a 125001 transport tank is used to fill them. The transport tank is mounted on a trailer and equipped with a bacteriological air filtering system, an under-pressure sensor, an over-pressure release valve, a pump to fill the tanks and a 2 in. bore sanitary hose. The end of the hose is connected to a bayonet, which has a valve to regulate water flow and a freely rotating cap that can be screwed to the liner opening. During transportation, the bayonet is protected with a stainless steel scabbard, which can be screwed to the bayonet with an airtight seal. The end of the bayonet has a small stainless disc to open the water flow, thus preventing a localised water pressure on the liner, which could damage it, or displace it from the correct position. The trailer with the transporter tank was pulled by a truck on main roads and on good tracks, and by a frontloaded vehicle in sandy and muddy areas.

Although the water plant is currently producing water of resistivity above $15 \mathrm{M} \Omega \mathrm{cm}$, it was producing lower quality water when the EA tanks were filled. All of them were filled with water having a resistivity between 1 and $7 \mathrm{M} \Omega \mathrm{cm}$, except for the two tanks that were filled first. For these, lower quality water (resistivity below $500 \mathrm{k} \Omega \mathrm{cm}$ ) from a local provider was used. The transparency of the water is monitored using the 
decay time of the signals from the PMTs. These two tanks, as well as a third, a test tank situated at the central campus, which is fitted with a re-used liner, have shown a faster decay time than observed in any of the other tanks. The present variability of the water resistivity allows study of the signal dependence on liner and water properties. The observed variation of signal shape and amplitude with water quality will provide a valuable diagnostic at later stages.

Water from some selected detectors is monitored periodically. The filling of detectors is not a sterile procedure and a very definite kind of bacteria was found in many of the detectors. Bacteriological analysis showed a number of colony-forming units of Serratia (an aerobial mesophyl). However, in all of the sampled detectors, the number of bacteria remained stable (below 2000 colonies $\mathrm{ml}^{-1}$ ) or decreased, over a period of more than 2 years. This is interpreted as indicating that the tanks do not provide an environment for bacterial growth.

\subsection{Electronics}

Except for the PMTs and their bases, all of the electronics is contained in a plastic waterproof box situated next to the antenna mast or below the solar panels. A schematic of the various boards used in the EA is shown in Fig. 3, along with a typical pulse from low-energy shower, as recorded at the end of the complete local acquisition chain.

\subsection{PMTS}

Three different types of PMTs were used in the EA: Hamamatsu R5912 (8 in. diameter), ETL 9353 (8 in. diameter) and Photonis XP1802 (9 in. diameter) as part of an evaluation procedure. Details of other tests have been given elsewhere [13]. Some tanks were equipped with three identical PMTs, while others had one of each type. The PMTs are optically coupled to the windows of the liner with GE or Wacker RTV silicone, encased in a black fez for light and humidity isolation, and connected to the junction box above the larger hatch cover. A control cable brings the necessary voltages to the base of the PMTs. The high voltage supply is located directly on the base, and controlled by a low voltage from the slow control board (see Section 3.7). Signals are extracted from the last dynode and from the anode. An amplification of $\times 32$ is applied on the dynode signal to match the dynamic range. The anode signal will be used for large signals, typically those seen by tanks relatively close to
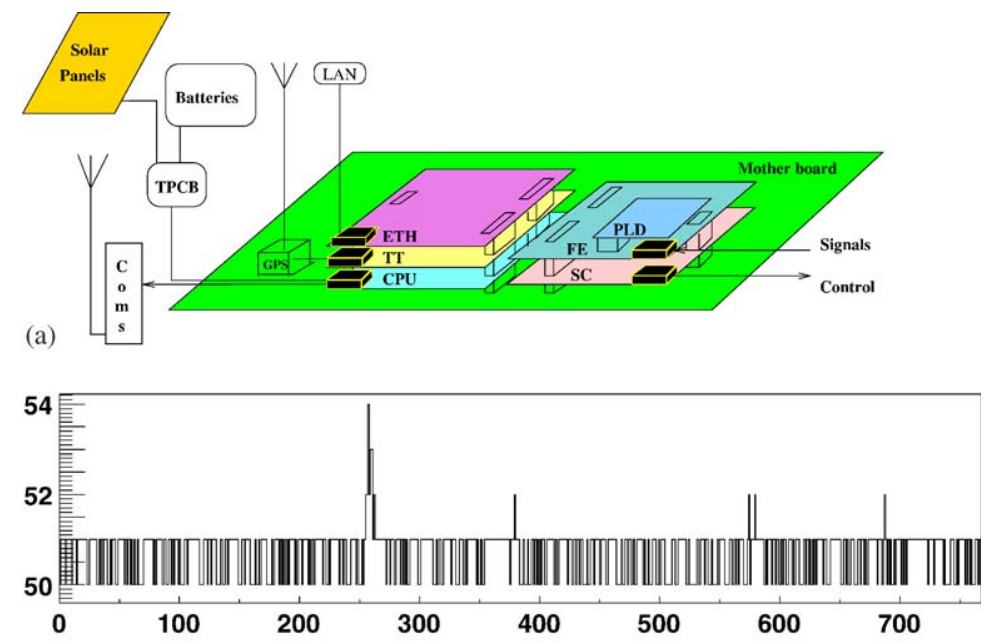

(b)

Fig. 3. (a) Schematic diagram of the electronics of the EA; (b) A typical signal from an FADC channel. Each time bin corresponds to one $40 \mathrm{MHz}$ clock tick, or $25 \mathrm{~ns}$. 
the core. The cross-over point corresponds roughly to the signal from a vertical event of $10^{19} \mathrm{eV}$ within $700 \mathrm{~m}$ of the core. Half of this amplification is done at the PMT base, with the other part being carried out at the front-end electronics (see 3.6).

Analysis of the data from the first 6 months of the operation of the EA (from July 2001) was used to determine which phototube would be used in the whole array.

\subsection{Front-end electronics and first level trigger}

The readout of the six signals from each tank (the signals from the anode and the amplified dynode of each of three PMT) is accomplished using front-end electronics having six 10 bit Fast Analog to Digital Converters (FADCs) running at $40 \mathrm{MHz}$. The digitised signals are sent to a daughter PLD (programmable logic device) board, which is used to implement the various triggering decisions.

Two different trigger modes are currently implemented in the EA for the first level (T1) trigger. The first uses a 'single threshold'. In this mode, one looks for a coincidence of some PMTs above a threshold. The second mode uses the 'time over threshold' in which a specified number of $25 \mathrm{~ns}$ bins are each above a threshold value within a pre-determined time window. A further trigger mode, based on the sum of the signals, can be programmed, but has so far not been used.

The majority of data from the EA has been taken using the 'single threshold' trigger set at a 3-fold coincidence of 1.75 Vertical Equivalent Muons, (VEM, see Section 3.9) on each PMT, and with the 'time over threshold' requiring a 2-fold coincidence of 12 bins at $0.2 \mathrm{VEM}$ within a $3 \mu$ s window. For this trigger, 12 of the 120 bins (a $3 \mu$ s window) must be above 0.2 VEM for two PMTs at the same time. Any of these parameters may be changed using the slow control system (Section 3.7).

Whenever a trigger is generated, a time block of $19.2 \mu \mathrm{s}$ (768 bins) from the FADC is copied to a buffer, which can be accessed by the station controller. There are 256 pre-trigger and 512 post-trigger bins. This first level trigger can operate at a maximum rate of $\sim 200 \mathrm{~Hz}$ but has been running routinely at around $110 \mathrm{~Hz}$.

\subsection{Station Controller}

The local electronics is controlled by a CPU board that hosts a Power PC 403GCX at $40 \mathrm{MHz}$ running under the OS9 operating system. The station controller is used to select from the T1 trigger signals, arriving at $\sim 110 \mathrm{~Hz}$, the ones likely to have come from EAS. Such events are forwarded to the CDAS system at the Central Station. For the EA, all 'time over threshold' triggers are designated as second level triggers (T2), as are any single threshold triggers above 3.2 VEM. These trigger choices mean that the efficiency of the array is greater than $90 \%$ above $10^{19} \mathrm{eV}$. The rate of T2 triggers is around $20 \mathrm{~Hz}$. A slow control board (SC) is used as the interface to the PLD trigger, so that thresholds and PMT voltages can be set, and a wide range of monitoring information can be read. The monitoring information is collected from a range of sensors that provide the temperatures of the electronic box and PMT bases, and from the motherboard voltages.

The time at which a local station triggers is crucial for determining the shower direction. It is measured at each local station using a commercial Motorola GPS board (OnCore UT). The signal is processed by the CPU board and a time tagging board which provides the event time with a precision of $\sim 8 \mathrm{~ns}$. To achieve this time resolution, precise knowledge of the position of each station must be provided to the CPU board. This position will be obtained routinely during the surveying procedures carried out as part of tank deployment. Most of the data from the EA has been taken without these coordinates being fixed, degrading the resolution to around $14 \mathrm{~ns}$. This timing precision, measured in the laboratory with respect to an atomic clock, has been replicated in the field.

\subsection{Power supply}

The electronics at each station is designed to have a power consumption lower than $10 \mathrm{~W}$ as 
solar power is used for regular operation. Two solar panels of nominal peak power between 51 and $64 \mathrm{Wp}$ (watts produced under standard conditions with $1000 \mathrm{~W} \mathrm{~m}^{-2}$ illumination) are connected to two $12 \mathrm{~V}$ batteries which are situated in a box in the shadow of the tank. A tank power control board (TPCB) is used to monitor the various voltages and currents of the system and to provide $24 \mathrm{~V}$ to the motherboard. A wooden box has been installed at all EA tanks to encase the cables from the batteries, thus preventing problems that were initially experienced with cows.

\subsection{Detector calibration}

To use the data from the detectors, the minimum calibrations that are required are for timing, the dynode to anode ratio, and the absolute calibration of the three dynode signals for each tank.

The timing calibration is derived from the GPS system and the time tagging board. Two tanks (known as Carmen and Miranda), separated by $11 \mathrm{~m}$, have been used to compare the timing accuracy achieved in the field with that measured in the laboratory. The two adjacent tanks are shown in Fig. 4a. Apart from the Carmen and Miranda pair, the coincidence rate between any two tanks of the surface detector is $\sim 8 \times 10^{-4} \mathrm{~Hz}$

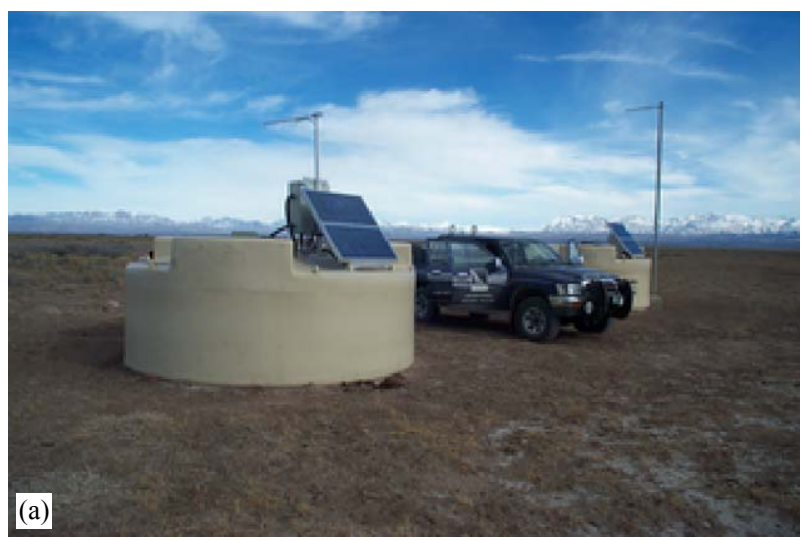

for a $1 \mu$ s window. The overwhelming majority of these are chance coincidences. The coincidence rate between Carmen and Miranda is approximately $0.8 \mathrm{~Hz}$. The majority of the CarmenMiranda triggers are the result of relatively lowenergy showers (from primaries of $\sim 10^{15} \mathrm{eV}$ ) falling close to the detector pair. The signals associated with these low-energy events have been recorded and are used to compare the timing measurement at each tank. A Gaussian profile of the time differences, with a rms spread of $17 \mathrm{~ns}$, has been obtained (Fig. 4b). This spread results from the convolution of the physical dispersion due to the separation of the two tanks (around $13 \mathrm{~ns}$ ), and the timing resolution (of each tank) and gives an estimate of the timing error of $\sim 8 \mathrm{~ns}$ sigma. Systematic, but stable, offsets of $<60 \mathrm{ns,}$ between different GPS receivers have been identified and measured in the laboratory.

Muons produced in the atmosphere provide the basis of the calibration chain. In addition to forming an extremely well-understood and uniform background across the whole of the surface array, the signal from a muon traversing a Cherenkov tank is proportional to the geometric path length. The calibration method developed aims to determine quantities related to the signals associated with muons. The peak due to single muons crossing the tank is clearly visible in a histogram of signals from a PMT (Fig. 5). The

Fig. 4. (a) The Carmen and Miranda tank pair; (b) timing difference for low-energy showers triggering the Carmen-Miranda pair. The $17 \mathrm{~ns}$ sigma Gaussian distribution corresponds to an individual resolution of $8 \mathrm{~ns}$ for each tank. The $50 \mathrm{~ns}$ offset is a stable offset that is characteristic of this receiver pair. 


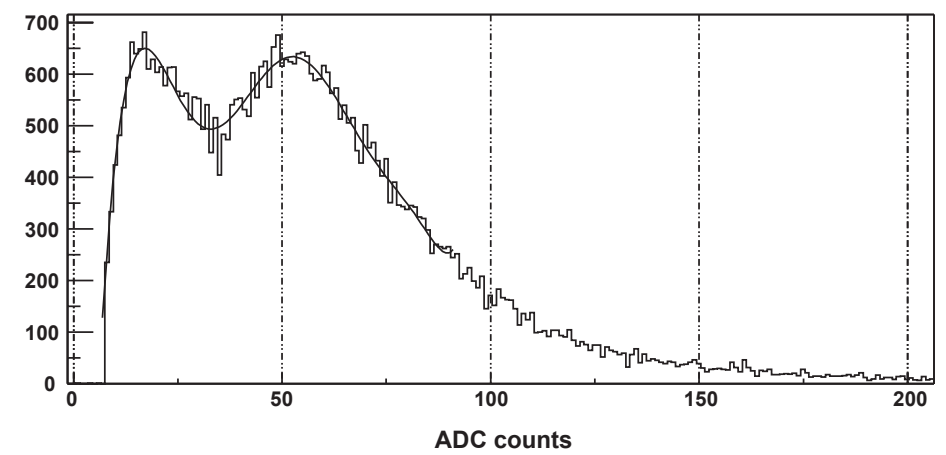

Fig. 5. Histogram of signals from one PMT in one of the stations of the EA. The peak due to single muons is clearly visible at around 50 ADC channels. The peak at about 20 channels is artificial and is due to the cut made in plotting the data.

position of this peak is an important calibration parameter and can be related to the average charge deposited by a high-energy down-going vertical and central muon, called a VEM. A calibration procedure has been developed to determine the peak position and its relation to the VEM, so that subsequent measures of the signal can be given in units of VEM.

PMT calibration is carried out in three steps. First, the three PM tubes are matched in gain by adjusting their voltages so that the rates above a common threshold are the same. Then, using an 'on-line calibration', the evolution of the gains is monitored and included in the data flow. Finally, the absolute calibration is determined from a sequence of measurements made on an identical test tank located at the Central Campus.

From the measurements made using the Campus detector, the rate of an energy deposit in an Auger tank above 3 VEM is known to be around $100 \mathrm{~Hz}$. Therefore, once a value in ADC counts is chosen for 1 VEM in a tank (50 channels in the EA), the trigger is adjusted so that for a specific PMT the threshold is 150 channels ( 3 VEM) above the baseline (a stable ADC pedestal). The voltage is then adjusted until the rate is $100 \mathrm{~Hz}$. Once tanks are calibrated using this method, one can compare the position of the muon peak from Fig. 5 with the expected value of 50 . The dispersion at the 3 VEM level is $\sim 8 \%$ and is sufficiently small to allow the next calibration step to be taken.

With the PM tubes approximately matched, a further refinement is made on-line, again using rates with the station in the data acquisition mode. As the first level trigger consists of a 3-fold coincidence above a specific threshold (1.75 VEM), PMTs that have drifted out of calibration can readily be identified by looking at the rates from individual photomultiplier tubes above a higher threshold (2.5 VEM). If all PM tubes are gain-matched, one expects all three to give the same rate $(70 \mathrm{~Hz})$. If one has a higher gain, the rate of the threefold coincidence will not increase significantly (as it is a 3 -fold coincidence), whereas the individual count of the PMT that is misaligned will be higher than expected. The local station controller can therefore be used to adjust an internal value of the VEM for each PMT, modifying the trigger levels until all PMTs have a similar rate above the 2.5 VEM threshold.

The signal in an event can peak just at threshold on any PMT. In this case, the dynode signal for such a PMT at the trigger bin is exactly at the threshold of 1.75 VEM, and the charge associated with it is computed for that PMT by integrating the signal over $625 \mathrm{~ns}$. As this dynode signal has a 1.75 VEM peak, it is expected that this charge will be close to that associated with 1.75 VEM. The charge associated with 1 VEM is thus extracted from the division of this value by 1.75 . Using the local controller, the VEM values associated with the peak and charge for each PMT are sent to CDAS along with the data of those events that are forwarded for reconstruction. Additionally, these VEM values are transmitted at 6 min intervals to 
allow performance monitoring. Every $4 \mathrm{~h}$, histograms are compiled, taking data at low threshold (0.15 VEM threefold) to compute the position of the muon peaks. The Gaussian dispersion of the VEM values was less than $2 \%$ for all 96 PMTs over the first year of implementation of this algorithm.

Whenever a signal is recorded above 8 VEM, but below 20 VEM, the dynode to anode ratio is computed at the station controller, both signals being integrated for $500 \mathrm{~ns}$. The parameters needed for the cross-calibration of signals from any station with respect to the muon peak are then available.

The final step is to relate the peak with the reference physical value, namely the signal associated with 1 VEM. This value has been measured using a muon telescope, with two scintillators, placed above and below the EA test tank situated at the Central Campus. Measurements have been taken using the internal station trigger and compared with external triggers produced by the muon telescope. These relations are integrated within the data reading software. Stations are calibrated with respect to this absolute value of the VEM with an overall precision of $5 \%$.

\section{Fluorescence detectors of the engineering array}

\subsection{Fluorescence detector}

A single fluorescence detector unit comprises six telescopes, each located in independent bays, overlooking separate volumes of air. Two prototype fluorescence telescopes of the EA were installed in bays 4 and 5 of the Los Leones building. A schematic cross-sectional view of one fluorescence telescope is shown in Fig. 6. A circular diaphragm, positioned at the centre of curvature of the spherical mirror, defines the aperture of the Schmidt optical system. UV transmitting filters are installed in the entrance aperture. Just inside the UV filter is a ring of Schmidt corrector elements. Light is focused by a large $3.5 \mathrm{~m} \times 3.5 \mathrm{~m}$ spherical mirror onto a 440 PMT camera, which accommodates the $30^{\circ}$ azimuth $\times 28.6^{\circ}$ elevation field of view. Each

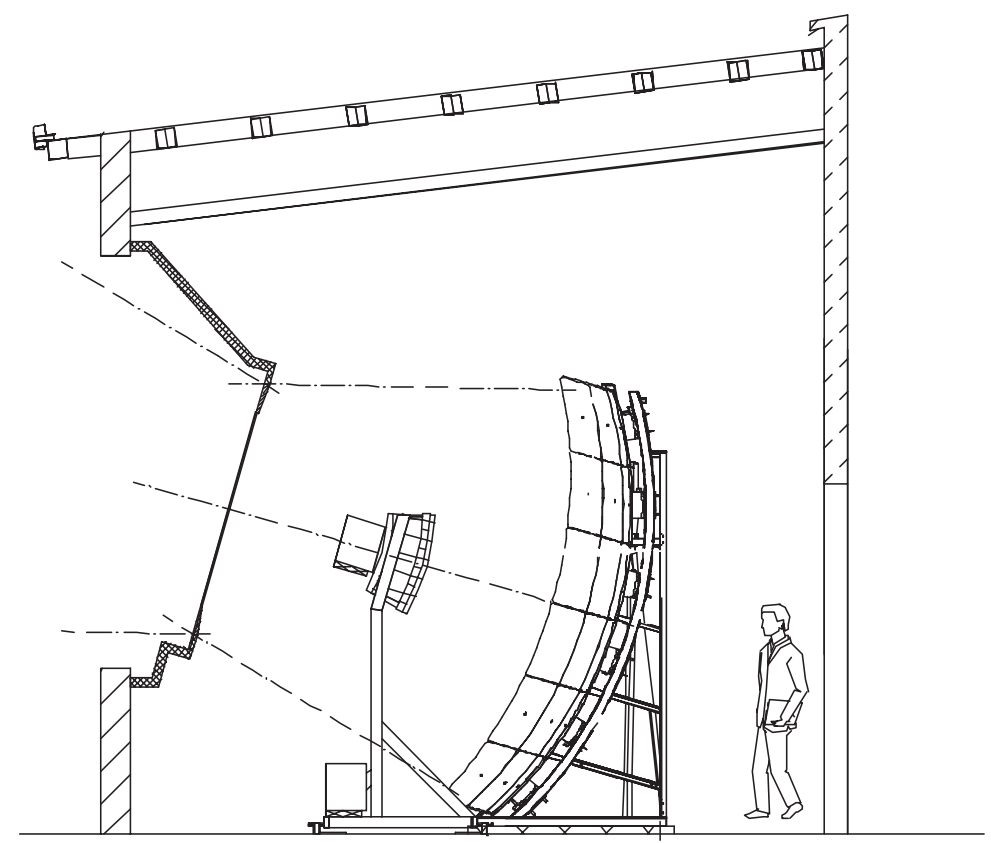

Fig. 6. Schematic view of a fluorescence detector telescope. From left to right can be seen the aperture system, the PMT camera and the spherical mirror. 
camera pixel has a field of view of approximately $1.5^{\circ}$.

\subsubsection{Optics}

The choice of Schmidt optics [14] gives the advantage of eliminating coma aberration: the circle of least confusion ("spot"), caused by spherical aberration, is practically independent of the incident direction. The diameter of the diaphragm was set at $1.7 \mathrm{~m}$, giving an effective area for light collection of $1.5 \mathrm{~m}^{2}$ after taking account of the shadow of the camera. The radius of the mirror is $3.4 \mathrm{~m}$ and the angular size of the spot from spherical aberration is $0.5^{\circ}$, i.e. $\frac{1}{3}$ of the pixel size.

The reference design of the aperture was upgraded in the bay 4 to increase the effective aperture by about a factor of 2 while preserving the quality of the spot [15]. This was achieved by the addition of a corrector ring, of annular shape, with radial extension of $25 \mathrm{~cm}$ (inner radius of $0.85 \mathrm{~m}$ and outer radius of $1.10 \mathrm{~m}$ ) that complemented the standard circular diaphragm of $0.85 \mathrm{~m}$ radius. For reasons associated with construction, the corrector plate was split into 24 sectors. The plate is made of UV transmitting glass, machined with an appropriate aspherical profile to compensate for the spherical aberration. This technique avoids an increase in spot size.

To improve the ratio of the signal to the noise from the sky background, a UV transmitting filter is placed on the diaphragm [16]. The filter transmits nitrogen fluorescence light while also acting as the window of the bay, thus protecting the telescope from the external environment. Sheets of M-UG6 glass (by Schott-Desag), $3.25 \mathrm{~mm}$ thick, are used. The filter transmits the nitrogen fluorescence spectrum in the near-UV efficiently, while blocking almost all visible light, which would increase the background noise. A simple, but robust, support structure holds the glass sheets of $80 \times 40 \mathrm{~cm}^{2}$ in place.

The spherical mirrors, which are approximately square, were constructed from segments of smaller size, using either hexagonal or nearly square shapes. The technical challenges of the construction of such large and thick mirrors motivated the development of three different techniques of mirror production for the EA.

The mirror segments for the telescope in bay 5 were produced by forming a warm and soft glass plate against a spherical mould cast in a special stainless steel alloy (slumping technique) [17]. The glass segments were coated under high vacuum with a layer of $200 \mathrm{~nm}$ of aluminum and anodised for protection. The $13.4 \mathrm{~m}^{2}$ spherical surface of the mirror was obtained by assembling 49 segments of approximately square shape.

One half of the mirror in bay 4 was built from 18 mirror segments also of approximately square shape, made from solid aluminum alloy sheets [18]. The production steps for a mirror segment involved the milling of a cast aluminum backing, with approximate spherical shape, to an effective thickness of $18 \mathrm{~mm}$ and a radius of $3.4 \mathrm{~m}$. A $3 \mathrm{~mm}$ AlMgSi 0.5 sheet was then glued to the backing, with an appropriate epoxy, at high temperature $\left(150^{\circ} \mathrm{C}\right)$ under high pressure. The edges of the $\sim 65 \times 65 \mathrm{~cm}^{2}$ segments, and a central hole, were precisely milled. The final milling of the reflective surface was carried out in a specialised commercial factory with high quality machines and diamond tools. The mirror surface was protected by electrochemical anodisation. The second half of the mirror for bay 4 mirror was built from 42 high-quality hexagonal glass mirror segments [18]. The rough shape of the mirrors was obtained by pressing high temperature $\left(1450^{\circ} \mathrm{C}\right)$ molten borosilicate glass into a mould. After annealing in an electric conveyer furnace, the segments were machined with the standard techniques of the optical industry (cutting, drilling, milling, grinding and polishing). The final hexagonal shape was cut from a circular mirror of radius $500 \mathrm{~mm}$. Standard vacuum equipment with an electron gun was employed for the deposition of the reflective aluminum layer, with final coverage with a protective $\mathrm{SiO}_{2}$ layer.

All the mirror segments were subjected to rigorous control of surface quality, and of their geometrical and optical parameters. The average reflectivity of the segments was found to be close to $90 \%$ between 300 and $400 \mathrm{~nm}$. The deviations of the mirror segments from the nominal value of 
the mirror radius were typically within $20 \mathrm{~mm}$. The quality of the mirror segments was tested by requiring that $90 \%$ of the light from a source positioned in the centre of curvature was reflected within a circle of radius $5 \mathrm{~mm}$.

Each mirror segment was supported by an adjustable mount, which allowed optimisation of the orientation of the segment. The mounts were clamped to a simple mechanical structure built from three nearly identical stands bolted to ground and connected by cross bars.

The alignment of each telescope was performed with respect to a removable reference point, placed on a strong and precise mechanical support, corresponding to the centre of curvature of the mirror. The mirror segments were positioned at the correct radius by using a laser distance-meter fixed at the reference point. Their orientation was adjusted to better than $0.2 \mathrm{mrad}$ by observing a laser beam reflected back to the reference point. A similar procedure was used for the positioning and alignment of the focal surface, with an overall alignment precision expected to be better than $0.1^{\circ}$.

The manufacturing techniques used for the mirrors of bay 4 , which provided the best optical quality, were selected for the production of mirror segments for the full FD.

\subsubsection{PMT camera}

Because of the symmetry of the optical system, the actual focal surface is spherical in shape. It is concentric with the mirror and has a radius of $1743 \mathrm{~mm}$, slightly larger than the standard focal length. The camera is an array of 440 hexagonal pixels arranged in such a way as to adapt to this spherical surface [19]. The pixels are not regular hexagons, and their shape and size vary slightly over the focal surface.

Each pixel has an eight-stage PMT tube (Photonis XP3062) with a $40 \mathrm{~mm}$ side-to-side hexagonal photocathode, complemented by light collectors. The photomultiplier array is made of 22 rows and 20 columns. The camera body was made from a single aluminum block. It consisted of an accurately machined plate, $6 \mathrm{~cm}$ thick and approximately square $(94 \mathrm{~cm}$ horizontal $\times 86 \mathrm{~cm}$ vertical) with inner and outer surface of spheri- cal shape. The PMT tubes were positioned inside cylindrical holes drilled through the plate. The camera body was held in place by a simple, but strong, two-leg support. Power and signal cables run inside the two legs of the support, thus preventing additional mirror obscuration.

The hexagonal shape of the PMT cathode ensures optimal coverage of the focal surface. However, some space between the PMT tubes is needed for safe mechanical packing. Moreover, the effective cathode area is smaller than the area corresponding to the glass envelope of the tube. To maximise light collection and guarantee a smooth transition between adjacent pixels, each PMT was surrounded by a simplified version of the classical 'Winston cones'. The 'cone' is realised by a hexagonal set of flat reflecting surfaces. The basic element of the light collector is a "Mercedes" star fixed at the vertex of three adjacent pixels. The reflective surface was obtained by gluing aluminised Mylar on the mercedes surfaces. Laboratory tests of the properties of the light collectors demonstrated their efficacy in recovering light at the photomultiplier borders [19].

Each PMT tube was equipped with a Head Electronics unit (HE), which provided highvoltage biasing, signal driving, a signal proportional to the night background light and a test pulse. The PMTs were biased by an active voltage divider, which has the advantage of dissipating very low power while providing gain stability, despite the varying level of night sky background light. The tubes, biased with the cathode grounded, were operated with a gain of about $4.4 \times 10^{4}$ for an average high voltage of $835 \mathrm{~V}$. A differential-input and balanced-output low-noise preamplifier was used to provide very high rejection to common-mode noise, as well as to drive the analogue signal into twisted pair cables up to the front-end board.

About 1000 PMT units of the first batch for the EA telescopes were individually characterised. A test system to perform the basic measurements of the gain, linearity, photocathode uniformity, spectral response and relative calibration in an automatic way has been developed [20]. 


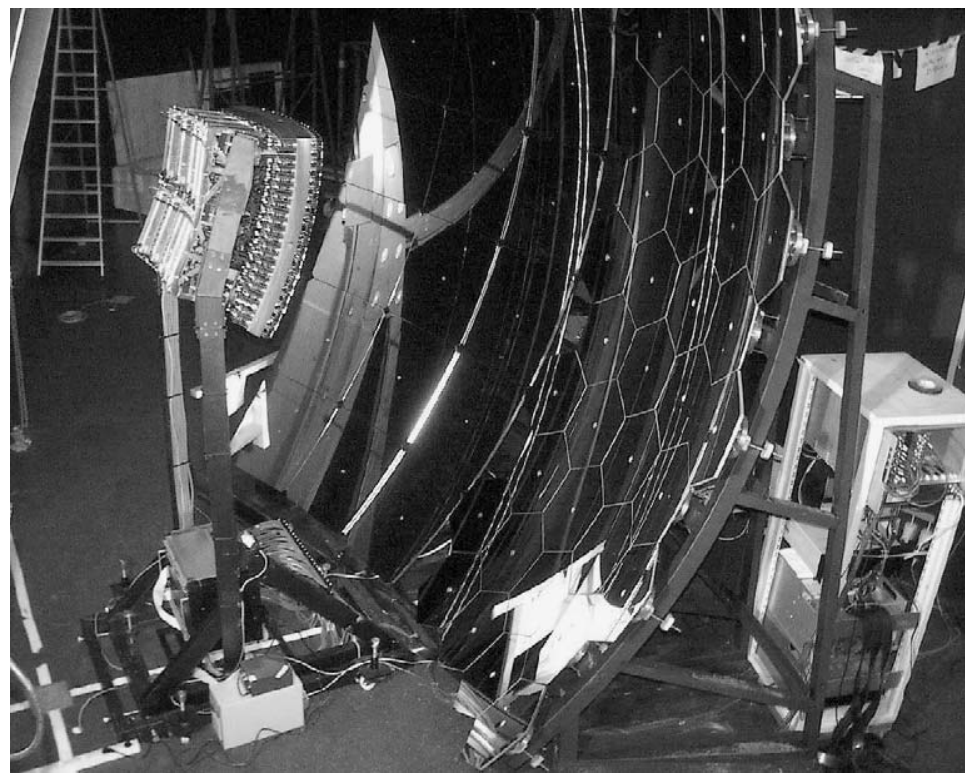

Fig. 7. FD telescope prototype installed in bay 4 at the Los Leones building.

The high voltage and the low voltage were distibuted by 10 PCBs positioned at the back of the camera, in its shadow region. The high voltage was provided by a CAEN SY527 system to 10 groups of 44 PMTs with similar gain. A commercial power supply with a distributor module provided the low-voltage bias. The differential signals from the HE driver were also grouped in the backplanes, and arranged in 20 cables, each one driving the signal of the 22 pixels of a camera column to one front-end board.

\subsubsection{Front-end electronics and first level trigger}

The PMT, signals are received by a set of 20 front-end boards [21,22] hosted in a crate placed on the floor below the camera. A photograph of the prototype telescope installed in bay 4 is shown in Fig. 7.

Each front-end board, serving 22 pixels of a camera column, provides proper treatment of the analogue signal from the PMTs with a differential line receiver, individual gain control and antialiasing active filter. The signals are then digitised continuously by $10 \mathrm{MHz} 12$ bit ADCs. Digitising two additional "virtual" channels, which are the analog the sum of the 11 odd and 11 even channel numbers at a lower gain, allowed a dynamic range of 15 bits to be obtained. Four sigma-delta ADC chips with 16-bit resolution read out the background light signal [21].

The digital part of the front-end board is used to implement all functions of the First Level Trigger (FLT) with reprogrammable FPGA logic. While the ADC values are continuously written to memory, the running sum of the last 10 samples is calculated. A pixel is marked as triggered, whenever the running sum exceeds an adjustable threshold. When the running sum again falls below the threshold, the pixel trigger is extended to a time of $20 \mu \mathrm{s}$, which is the overlap coincidence time common to all pixels. The trigger rate of individual pixels is measured continuously and kept close to a reference value of $100 \mathrm{~Hz}$ by automatic adjustment of the threshold. In this way, the random coincidence rate of the Second Level Trigger (SLT) remains constant under variable light background conditions. The number of samples of the running sum, the trigger time overlap and the reference trigger rate are programmable, allowing high flexibility during the EA commissioning. The trigger FPGA also implements the calculation of the pixel ADC variance, which is proportional to the DC background light. 


\subsubsection{Second level trigger}

The SLT FPGA logic is implemented on a separate board, which is used to read the pixel triggers generated for each channel in the 20 FLT boards. The SLT algorithm is used to search for patterns of five pixels consistent with a track segment, and to generate an internal trigger for data readout. Synchronisation with the front-end board is provided by a GPS clock. The algorithm makes use of the fundamental patterns shown in Fig. 8, as well as those corresponding to rotation and mirror reflection of these segments. The algorithm requires only four triggered pixels out of the five pixel track segment, allowing for possible faulty PMTs or small signals below the FLT threshold. The total number of different pattern classes is 108 .

The SLT FPGA is used to read the pixel trigger of a camera column into a pipeline of five stages every $50 \mathrm{~ns}$. The pipeline holds the image of a $22 \times 5$ submatrix of the camera. A pattern algorithm uses coincidence logic to search for the allowed patterns inside this submatrix while the image is shifted, column-by-column, over the full camera. The full camera image is scanned by the SLT module in $1 \mu \mathrm{s}$. If the coincidence condition is fulfilled for any of the pattern classes, the recording of ADC data is continued in the next free circular buffer, and the Mirror PC is informed. Typical SLT random coincidence rates of a few tenths of $\mathrm{Hz}$ per telescope were measured during the period of EA operation.

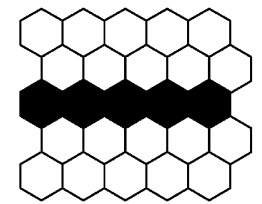

Type 5_0

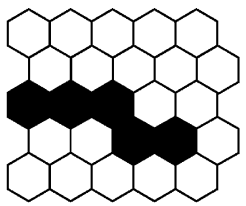

Type 3_2

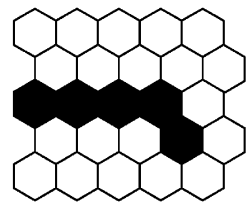

Type 4_1

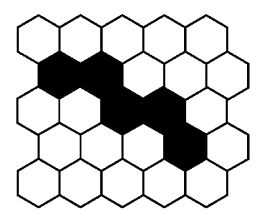

Type 2_2_1
Fig. 8. Basic topological patterns of triggered pixels used in the second level trigger.

\subsubsection{Data acquisition system and slow control}

The Mirror PC, a robust, diskless, industry PC associated with each telescope, is used to perform the data readout and a simple software, third level, trigger selection, based on track length and spacetime compatibility requirements. A more sophisticated T3 algorithm is also implemented, which selects shower candidates and performs a fast reconstruction of the shower geometry. All pixels that are part of a SLT of the event are selected. A fast search for a maximum of the ADC trace around the FLT time of each pixel is performed. A large part of the background, which is due to cosmic rays passing through the PMTs of the camera, is rejected by requiring that the selected pixels do not fire at the same time. Fits of the pixels to the elevation angle vs. azimuthal angle and time are performed, and pixels with a large contribution to the $\chi^{2}$ fit are removed. After this procedure, candidates with more than four pixels are considered good showers, and the azimuth of the shower impact on ground, as well its time of arrival, are calculated. For good showers, a T3 signal is sent to the central data acquisition system (CDAS), together with information on the shower-detector plane and the time of the shower arrival at ground. These data are then used to form the hybrid trigger. The observed rate of good showers satisfying the T3 trigger was about $5 \mathrm{~h}^{-1}$ per telescope during the period of EA operation. The DAQ system was developed under the Linux operating system in the $\mathrm{C}++$ language. An event display and online histograms were available to monitor data quality. An example of shower candidate as seen in the online event display is shown in Fig. 9.

A slow control system is used to guarantee that the FD operates only under safe conditions. Opening of the external shutters is prevented during daylight or during windy weather. To avoid direct exposure of the PMT cameras to sunlight in the case of shutter failure, a fail-safe curtain is installed at the aperture. The high voltage of the camera PMTs is shut down when any high light level is detected inside the bay. An uninterruptible power supply (UPS) protects the operation of the computers and shutter should there be a failure of mains power. 


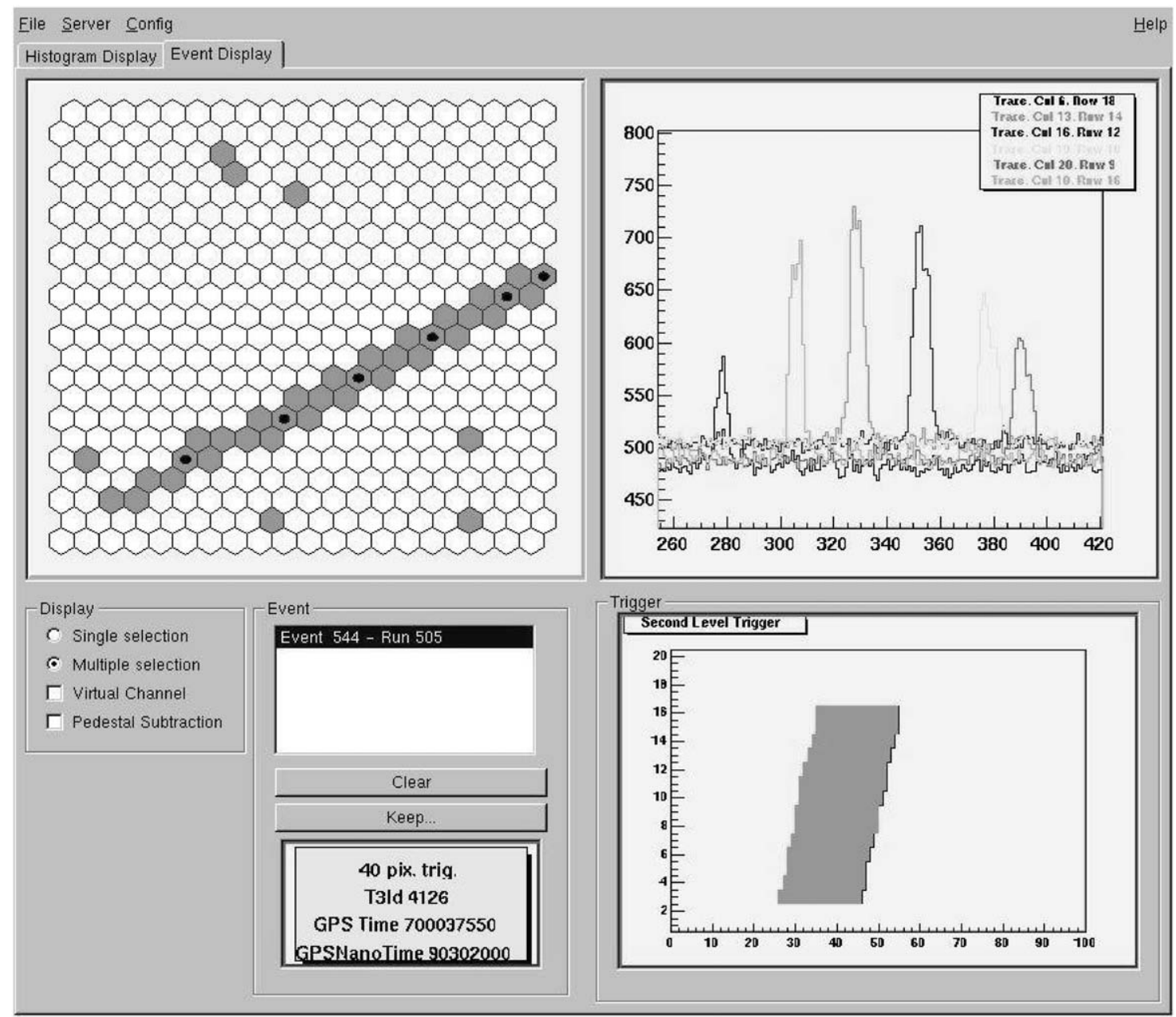

Fig. 9. On-line event display of a shower candidate. In the upper left diagram, the pixel matrix with triggered pixels is shown. The pixels with a black dot are selected for the ADC view. In the upper right diagram, the ADC values of the selected pixels are shown as a function of time in $100 \mathrm{~ns}$ bins. The lower right diagram shows the trigger intervals for each camera row as a function of time in bins of $1 \mu \mathrm{s}$. The time development of the shower is clearly visible.

\subsubsection{Monitoring of the DC light levels}

Although the entire electronic system is designed to optimise the sensitivity to short PMT pulses, it is also necessary to determine the average DC light level for several reasons:

1. Protection of the PMTs against excess light of artificial (vehicles, aeroplanes) or natural (rising sun or moon) origin to avoid destruction or accelerated ageing.

2. Knowledge of the sky brightness on a pixelto-pixel basis may be used to determine atmospheric conditions for the event reconstruction.

3. Tracking of stars across the camera is used to verify the absolute pointing of the telescopes and the long-term stability.

4. Optimal trigger conditions depend strongly on the light level at each pixel.

The EA telescopes are therefore equipped with two systems to determine the DC light level. These use a current monitor and fluctuation analysis. In the current monitor, a novel opto-coupled 

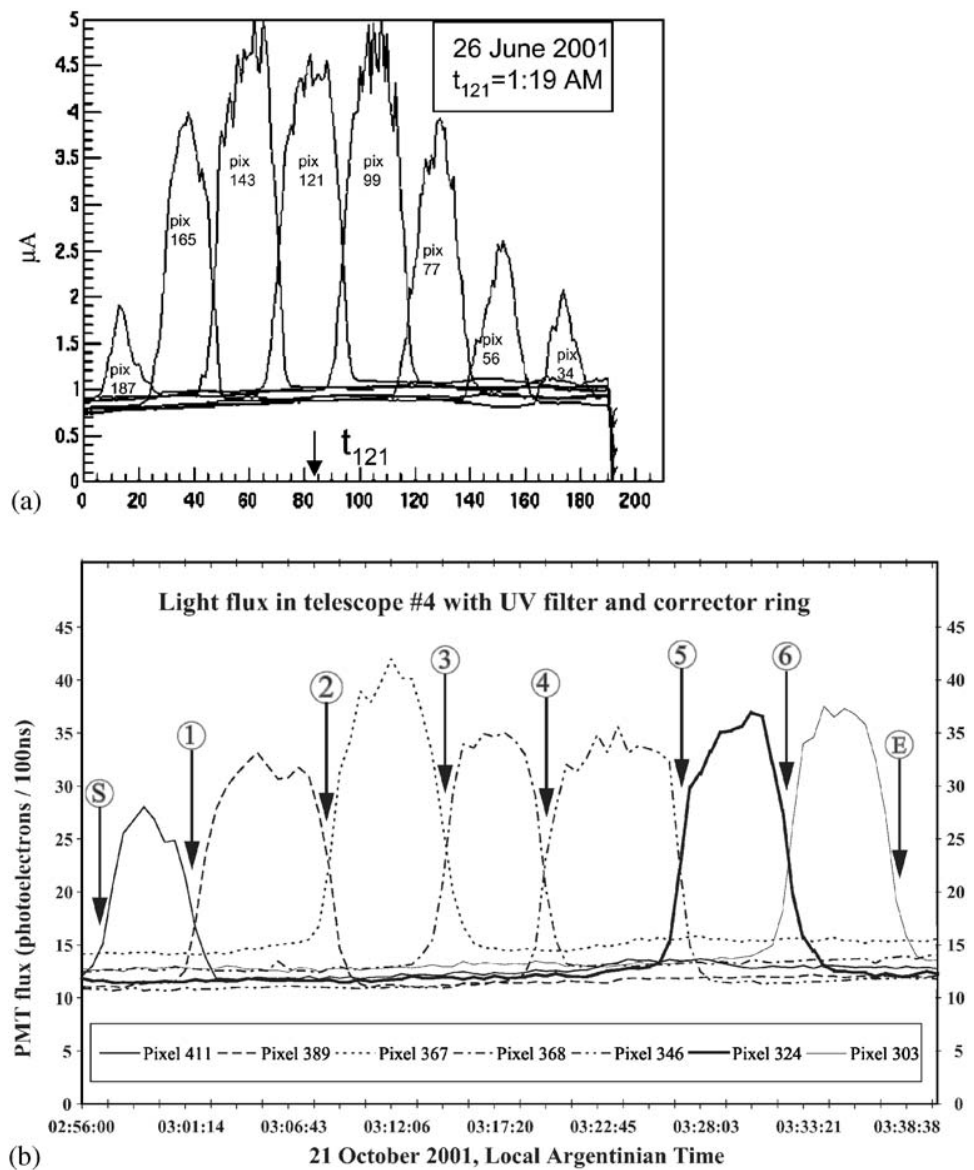

Fig. 10. (a) Signals of the current monitor for adjacent pixels vs. time. The DC light of the star alpha Lyrae induces peaks in the light level of individual pixels while it transits the FOV of the camera. (b) Light intensity determined from the statistical analysis of several camera pixels as a function of absolute time. The arrows indicate the times the light spot of the star Elnath transits from one pixel to another.

feedback-loop on the HE generates a signal proportional to the DC component of the anode current [21]. This signal is transported to the analogue section of the front-end boards and read using the sigma-delta ADC chips [21]. The fluctuation method uses the ADC variance as a measure of DC background light [23]. Both methods were tested with DC light from stars traversing the field of view of the camera. The track of the star alpha Lyrae and Elnath in the camera plane leads to peaks in the light level of adjacent pixels, as is shown in Fig. 10a and b, respectively.

With these methods it is possible to find light peaks from individual stars up to 7 th optical magnitude, depending on atmospheric conditions and the colour index of the star. Both methods of determining the DC light level gave similar results, but only the fluctuation analysis will be implemented in the final version to save costs and simplify the design.

\subsubsection{Detector calibration}

The precise reconstruction of air shower longitudinal profiles requires the conversion of an ADC count to a light flux for each pixel that receives a portion of the signal from a shower. To this end, the absolute calibration of the detector response is essential. A drum illuminator and Rayleigh calibrations provided an absolute, endto-end, calibration of the fluorescence telescopes. 
For these absolute methods, the flux of photons on the telescope aperture is independently measured and known to an absolute precision of about $10 \%$. The effects of diaphragm area projection, optical filter transmittance, mirror reflectivity, pixel light collection efficiency and area, cathode quantum efficiency, PMT gain, pre-amp and amplifier gains, and digital conversion are all included in the endto-end procedures. The drum illuminator consists of a pulsed UV LED, emitting in a narrow band around $375 \mathrm{~nm}$, embedded in a small cylinder of Teflon, illuminating the interior of a $2.5 \mathrm{~m}$ diameter cylindrical drum, $1.4 \mathrm{~m}$ deep [24]. The sides and back surfaces of the drum are lined with Tyvek, while the front face is made of a thin sheet of Teflon, which transmits light diffusively. The drum was positioned at the entrance aperture of the telescope under calibration. It provided illumination, uniform to within $3 \%$, over the entrance aperture of the telescope. Photographs of the drum illuminator are shown in Fig. 11. A NISTcalibrated photodiode was used to measure the absolute light flux to a precision of about 7\% before each telescope calibration. Absolute calibration constants were obtained from the known pulsed flux of photons emitted by the drum, and the corresponding ADC pulse integrals of the camera pixels.

For the Rayleigh calibration [25], a $355 \mathrm{~nm}$ laser was positioned a few kilometers from the fluorescence telescope to be calibrated. The laser was directed near-vertically. The laser beam was

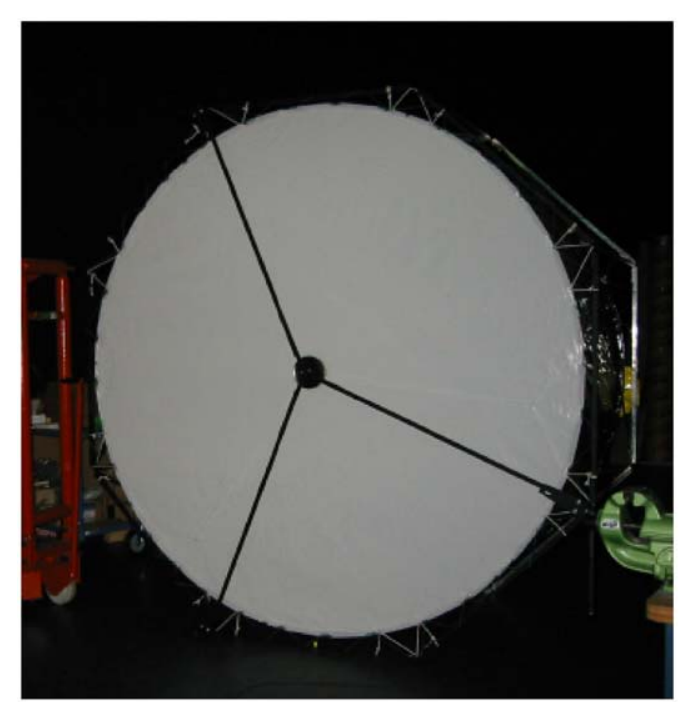

(a)

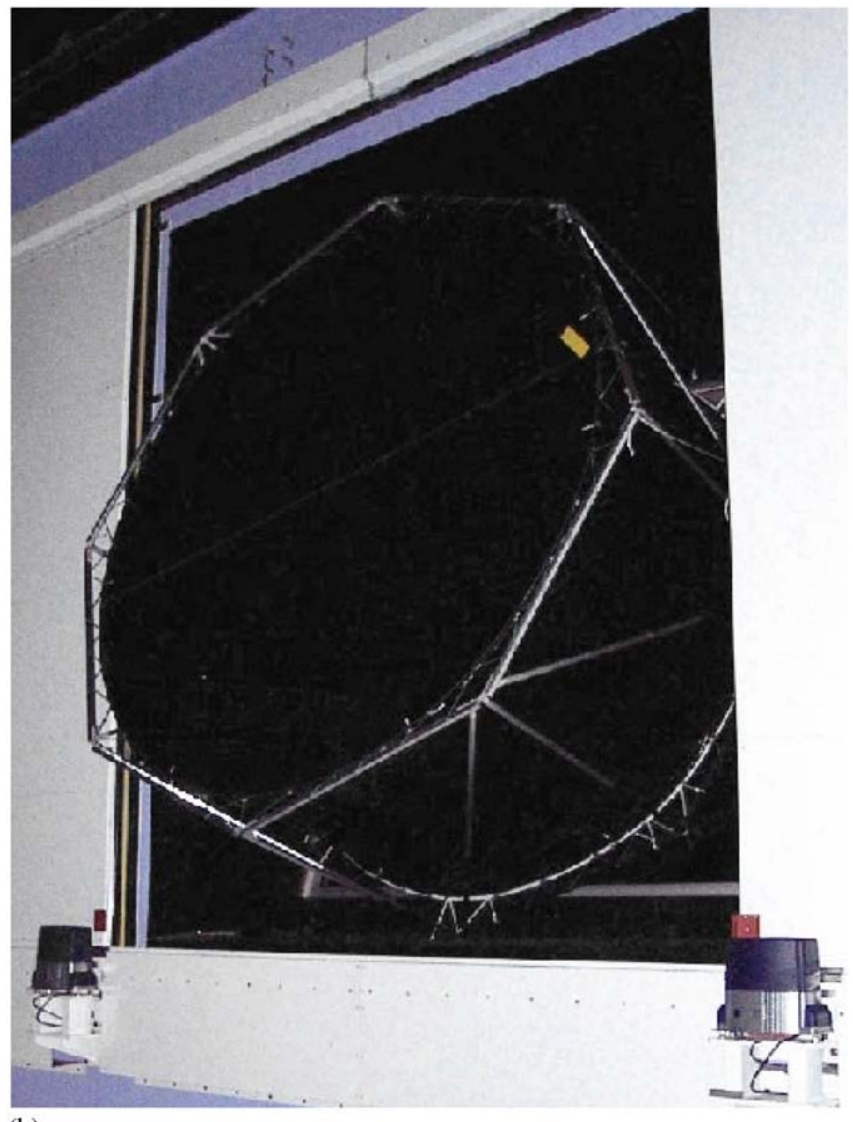

(b)

Fig. 11. (a) Front surface of the drum showing the Teflon sheet and light source in the centre. (b) Drum mounted at the telescope aperture. 
depolarised and the pulse-to-pulse intensity monitored to a precision of $5 \%$. The scattered light, mainly from Rayleigh scattering by the molecular atmosphere, was then used to calibrate the fluorescence telescope.

From the end-to-end calibration, the appropriate constants are found to be 4.5 photons/ADC count for bay 4 and 6.3 photons/ADC count for bay 5 . These differ because the fractional obscuration is larger in bay 5 than in bay 4 . To derive a flux of photons, the ADC number is multiplied by this constant, and divided by the area of the aperture $\left(3.80 \mathrm{~m}^{2}\right.$ for bay 4 and $2.27 \mathrm{~m}^{2}$ for bay 5$)$ and by the cosine of the off-axis angle. The latter division is the correction for the projected area when arriving light makes an angle with the telescope axis. The photon flux, in photons $\mathrm{m}^{2}$ per $100 \mathrm{~ns}$, perpendicular to the arrival direction is thus obtained.

An optical system for relative calibration [26] was used to monitor time variations in the calibration of the telescopes during the period of EA data taking. Three xenon flash lamp light sources coupled to optical fibres distributed light signals to three destinations on each telescope. Signals from one of the sources were brought to the centre of the mirror with the light directed to the camera. A second source was placed at the centre of two sides of the camera with the light directed at the mirror. The corresponding optical fibres terminated in $1 \mathrm{~mm}$ thick Teflon diffusers. The signals from the third source were sent to ports on the sides of the entrance aperture where the light was directed at reflective Tyvek targets mounted on the telescope doors from which it was reflected back into the telescopes. Drifts of the temporal performance of pixels, mirror and aperture components can be identified by comparing measurements from the three light sources. The sources were also equipped with neutral density filters to permit linearity measurements, or with interference filters to monitor stability at wavelengths in the range of $330-410 \mathrm{~nm}$.

\subsubsection{Atmospheric monitoring}

A precise determination of the fluorescence light emitted by the cosmic ray shower must take into account the attenuation of the light in its passage from the emission point to the detector. A detailed knowledge of the scattering properties of the atmosphere and its time dependence is mandatory. The programme of atmospheric monitoring, summarised in Ref. [27], includes a variety of complementary measurements. A fully steerable LIDAR system, located close to the FD building at Los Leones, was operated during the EA period. The system included a frequency tripled $\mathrm{Nd}: \mathrm{Yag}$ laser emitting pulses of $6 \mathrm{~mJ}$ energy and $7 \mathrm{~ns}$ duration at a wavelength of $355 \mathrm{~nm}$, and three $80 \mathrm{~cm}$ diameter parabolic mirrors to focus the back-scattered laser light onto a PMT tube. Due to the off-axis set-up, the laser beam is fully contained within the mirror field-of view when it is located more than $\sim 1 \mathrm{~km}$ from Los Leones. The signal was acquired using a $40 \mathrm{MHz}$ Licel digitiser connected to a remotely controlled DAQ system. The steering mechanism allows the atmospheric profile to be measured in any direction. Consecutive measurements at fixed elevation angles were performed over 5-10 min intervals to collect a statistically significant data sample. Fast scans, lasting for $10 \mathrm{~min}$ and continuous in elevation, were also made to monitor the stability of the atmosphere as a function of time.

To a reasonable approximation, the atmosphere is horizontally invariant above the Pampa plane. Thus the equation describing LIDAR return is solved with two- and multi-angle methods for the atmospheric optical depth $\tau$, affecting the attenuation of light, $T(r)=\mathrm{e}^{-\tau(r)}$, from distance $r$. The attenuation lengths, $(\mathrm{d} \tau / \mathrm{d} h)^{-1}$, obtained at ground level, are in the range $12-18 \mathrm{~km}$ and agree with horizontal attenuation measurements (HAM) made at the same site. The current LIDAR system allows the optical depth profiles to be assessed in the horizontal direction up to $25 \mathrm{~km}$, and at elevations up to $20 \mathrm{~km}$. Preliminary analysis has demonstrated that the atmospheric conditions are extremely clean, with variations below $2 \%$ over $30 \mathrm{~km}$ of horizontal distance, where the attenuation can be measured with a statistical accuracy of $\sim 1 \%$. The final systematic errors due to the attenuation coefficient are expected to be well under $10 \%$. An example of a typical data set is shown in Fig. 12. 


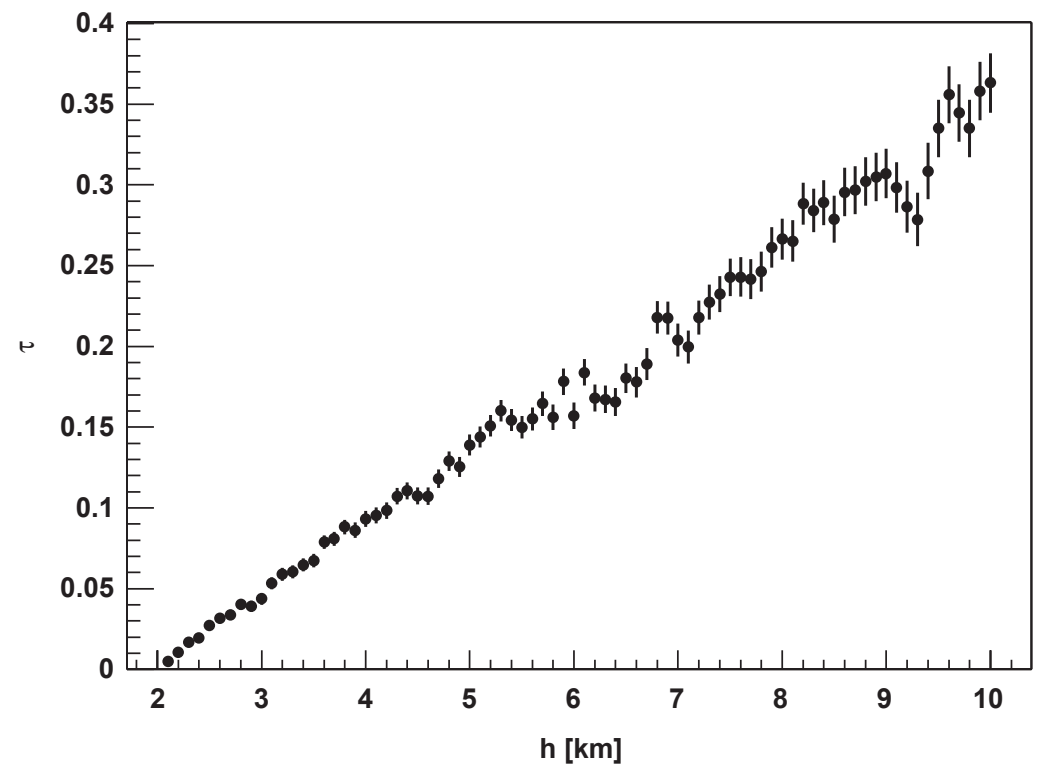

Fig. 12. The vertical equivalent of the optical depth $\tau(h)$ obtained from LIDAR measurements made in April 2002. In the altitude range $2-5 \mathrm{~km}$, the vertical optical depth $\tau(h)$ corresponds to an average attenuation length of $\sim 20 \mathrm{~km}$ at a wavelength of $355 \mathrm{~nm}$.

In addition to the calibration services for the fluorescence detector, the relative backscattering coefficients acquired from the same LIDAR measurements, in an aerosol-free atmosphere, provide an alternative estimate of the vertical air density profile. This is needed for the cosmic-ray shower reconstruction, as well as for Monte-Carlo simulation. Preliminary analysis shows that according to the US-standard atmosphere model, the aerosol contribution to backscattering is negligible above a height of $3 \mathrm{~km}$. Above this height, the LIDAR signal is expected to originate mostly from the Rayleigh scattering and is therefore a measure of atmospheric density.

Preliminary studies of the temperature and pressure profile in the vertical direction have been initiated using radiosonde balloons [28].

\section{Data communication system}

The data communications system of the Observatory consists of two integrated radio networks organised as a two-layer hierarchy. One network, the microwave network, is of high

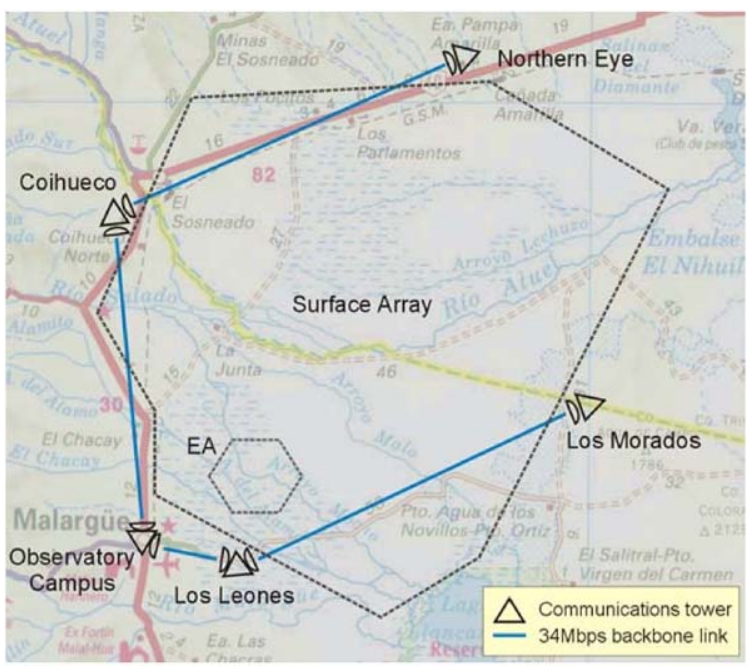

Fig. 13. The microwave network of the communications system.

capacity and provides communication from the FD sites to the central campus. It also supplies a series of distributed concentration nodes for data that are sent to and from individual surface detectors via the second network, a wireless Local Area Network (LAN). 


\subsection{Microwave network}

The microwave network uses a standard telecommunications architecture based on commercially available microwave equipment to provide point-to-point links. It operates in the $7 \mathrm{GHz}$ band. The equipment consists of microwave transceivers that are dish-mounted on communications towers, together with interface units located in shelters at the base of each tower.

Each microwave link supports a capacity of 34 Mbps. The EA uses one such link between the Observatory Campus and the Los Leones site. The full microwave network will consist of four links arranged as shown in Fig. 13. The configuration has two 'arms', one servicing both Coihueco and the Northern site and one servicing the sites at Los Leones and Los Morados. Both arms terminate at the Observatory Campus, where the data are routed to the Central Data Acquisition System (Section 6).

The microwave network has sufficient capacity for the transfer of the surface detector wireless
LAN data and for all data generated at the fluorescence detector sites. A generous margin has been included in the capacity requirement to permit support of future upgrades to the Observatory.

\subsection{Surface detector wireless $L A N$}

\subsubsection{Overview}

The wireless LAN that is used to communicate with the surface detectors has been designed for the project using custom radio hardware. Proprietary protocols for network access run on the hardware. The network operates in the 902$928 \mathrm{MHz}$ industrial, scientific and medical (ISM) band, providing data communications to and from each of the 1600 surface detectors. This is achieved in a manner similar to a cellular telephone system, whereby the area containing the detectors is divided into a number of sectors with communications within each sector co-coordinated using a base station. The requirement to use sectors is set by legislation relating to maximum

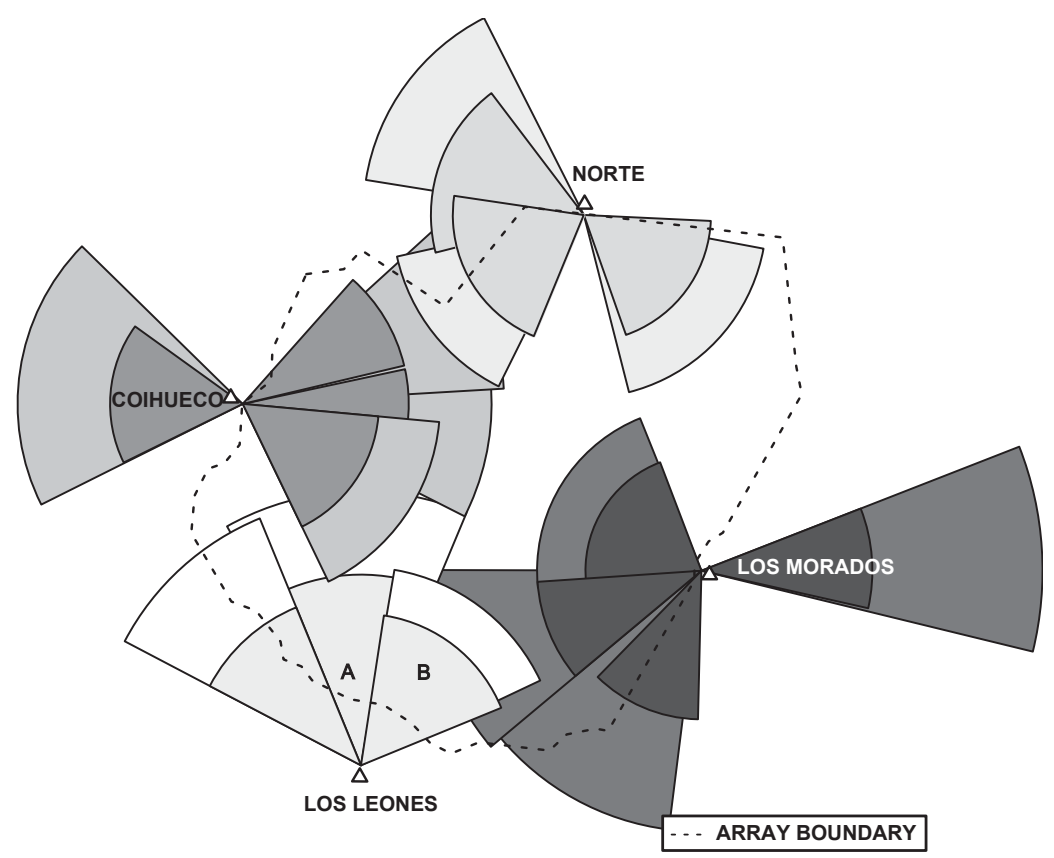

Fig. 14. Radio coverage of the 1600 surface array detectors is divided into 28 wireless LAN sectors, which each contain up to 60 detectors. A schematic approximation of the sector layout is shown which covers the full surface array area from 4 towers located at the edges of the array. 
transmitter powers and to frequency re-use within the ISM band. The use of sectors also enables an effective distribution of the processing load and reduces the possibility that a failure at a single node will cause data loss across the complete Observatory.

A simplified plan of the sector structure adopted is shown in Fig. 14. The base station antennas serving each sector are mounted on the communications towers: each tower supports up to eight sectors. The antennas are divided into two sets: an upper set mounted at the top of the tower and a lower set a few metres below. The upper set services sectors towards the centre of the array, whilst the lower set services sectors towards the array boundary. The wireless LAN will eventually contain 28 sectors. Typically, a single sector will service $57 \mathrm{SDs}$, although a single base station can serve up to $65 \mathrm{SDs}$ if required. The stations of the EA are supported by two base stations at the Los Leones tower: one is used to service tanks in sector ' $A$ ' and the other, tanks in sector 'B' (Fig. 14).

\subsubsection{Wireless $L A N$ subscriber unit}

Each surface detector is equipped with a wireless LAN subscriber unit (Fig. 15). This unit is interfaced with the surface detector electronics and is used to send trigger data back to an assigned base station using a time-division multiple access (TDMA) radio access protocol. The protocol is designed to guarantee that each surface detector has access to the network during each second. This custom-designed unit is a low-power design. It incorporates a digital radio transceiver that is software based and is mounted on a fourlayer printed circuit board (PCB) inside a nickelplated aluminium enclosure. For high reliability, every unit is 'burned-in' and each is calibrated over a temperature range of -15 to $+55^{\circ} \mathrm{C}$. Additionally, every unit has built-in self-test features and can be reprogrammed remotely from the central campus, thus facilitating network maintenance. This reprogramming capability was successfully demonstrated during deployment of the EA radio system when a late software change had to be introduced.

The processing functions of the subscriber unit are performed on a single 16-bit fixed-point digital signal processor (DSP). The subscriber unit firmware is stored on flash RAM and loaded into the DSP program memory when the unit is powered up.

The modulation scheme adopted for the radio transceiver is differential quadrature phase-shift keying (DQPSK), running at $10^{5}$ symbols s$^{-1}$. This corresponds to an 'over-the-air' bit rate of $200 \mathrm{kbits} \mathrm{s}^{-1}$ [29]. This data rate is shared between all subscribers within a given sector and hence supports a data payload of 1200 bits s$^{-1}$ for each subscriber unit of an SD. Data for error control and network monitoring are added using the communications protocol.

The subscriber unit consumes less than $1.0 \mathrm{~W}$ of power at $12 \mathrm{~V} \mathrm{DC}$, which meets the power budget requirements of the surface detector. A block

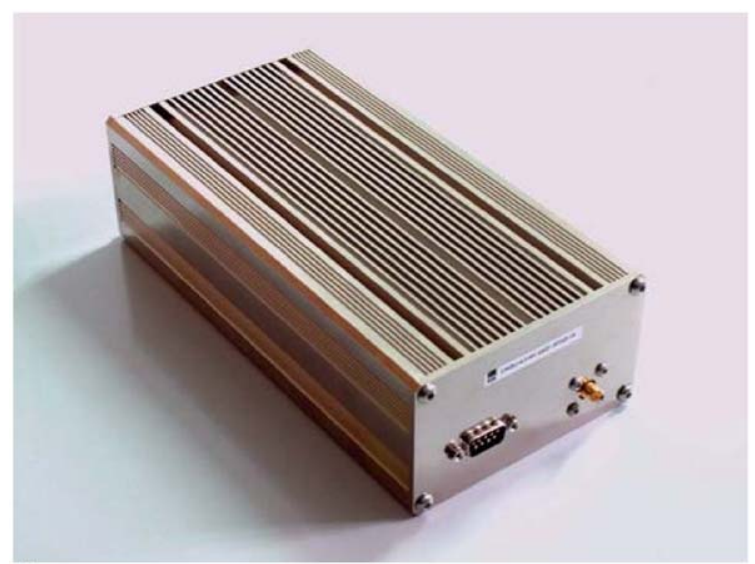

(a)

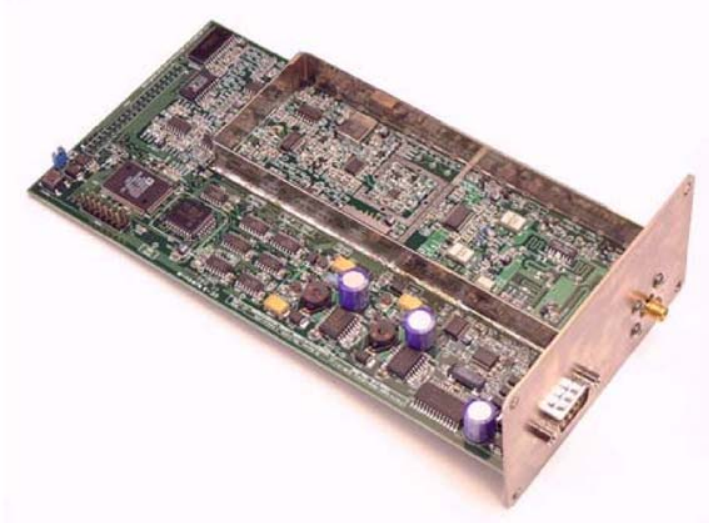

(b)

Fig. 15. Wireless LAN subscriber unit. 


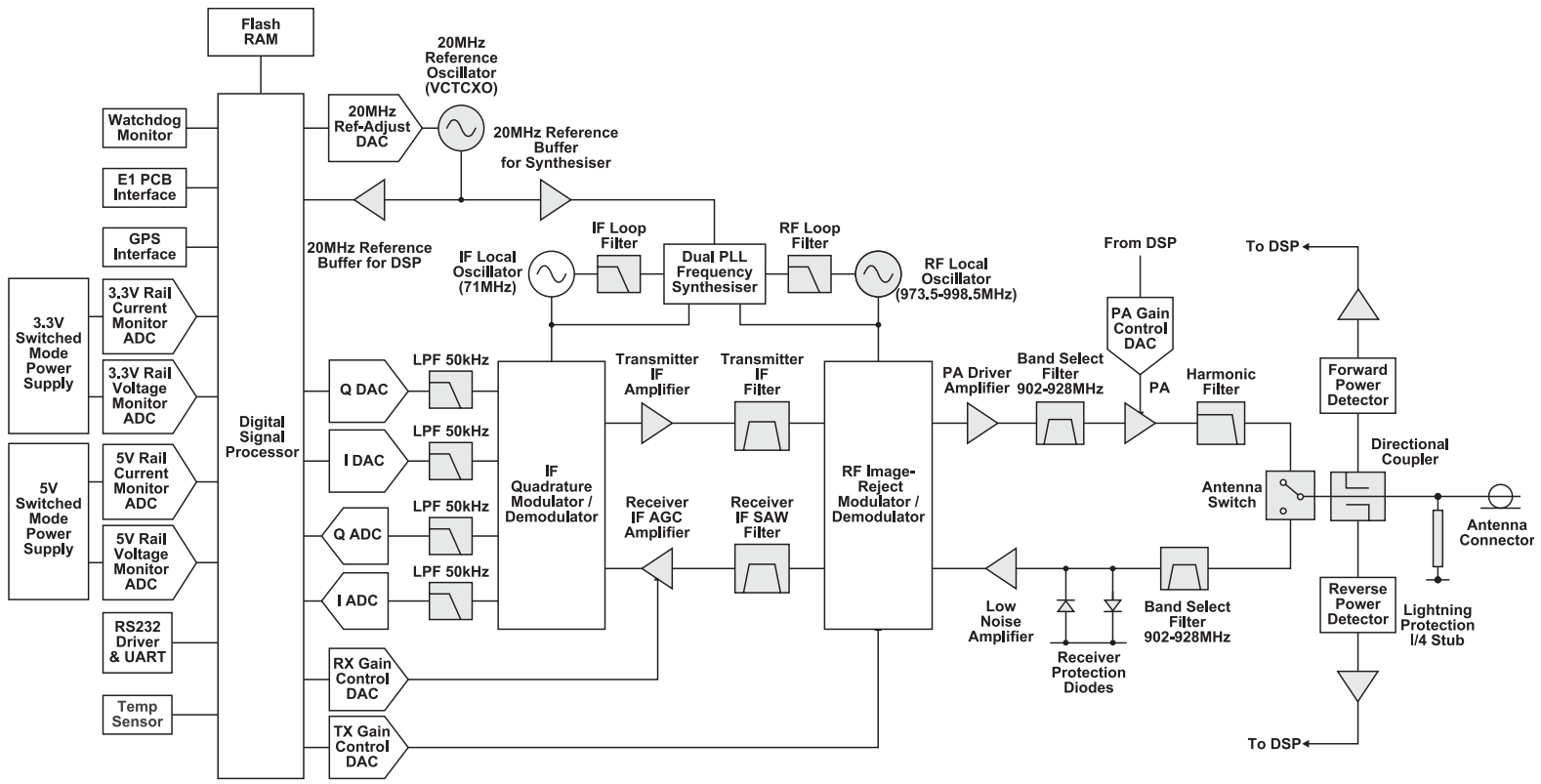

Fig. 16. Block diagram of the wireless LAN subscriber unit system.

diagram of the subscriber unit radio is shown in Fig. 16.

A radio section identical to that in each subscriber unit is used in the base-station radio. It is augmented by two additional DSP processors to accommodate the higher data load demanded at the base-stations.

\subsubsection{Wireless LAN antennas}

Two designs of antenna have been employed in the wireless LAN network. At each surface detector, where ease of installation and cost are important considerations, a $70 \mathrm{~cm}$ Yagi antenna is used. This has a beamwidth of $30^{\circ}$, which means that alignment to the communications tower is not critical, while retaining the gain at $12 \mathrm{dBi}$. The Yagi antenna is mounted on a short mast, integrated within the solar panel support at a height of $3 \mathrm{~m}$ above the ground (Fig. 2b).

The antenna requirements for the base station are somewhat different. To service an entire sector, the beamwidth needs to be wide, ideally greater than $60^{\circ}$, yet the antenna gain must still be sufficiently great to permit a higher value for the maximum allowable path loss and hence the

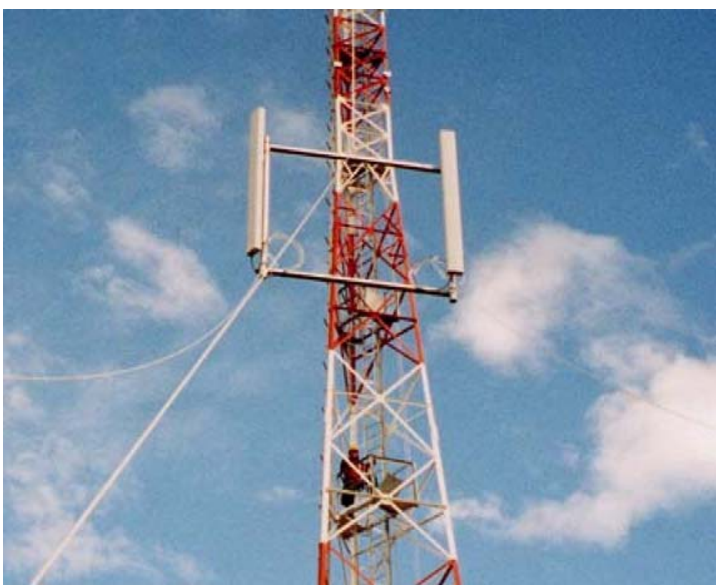

Fig. 17. Base station panel antennas being installed at Los Leones.

longer range radio links. To this end, commercial antennas, as used in mobile phone base stations, have been used at the towers. The antennas are $3 \mathrm{~m}$ high and are mounted at heights between 20 and $50 \mathrm{~m}$. The antennas are of a 'co-linear panel' type design and have a very high relative gain of $17 \mathrm{dBi}$ combined with a wide beamwidth of $90^{\circ}$ (Fig. 17). 


\subsubsection{Wireless LAN radio path}

Although the position of each concentrator tower has been dictated largely by the locations of the FD camera buildings, extensive simulations of path loss from each tower position to any point within the surface detector array have shown that full coverage of the site is possible with sufficient elevation of the antennas. These simulations were based on standard radio propagation models and digital elevation terrain data extracted from maps of the Observatory site. The integrity of the models was verified using field data generated in a longterm measurement campaign that began in September 1999.

The tower heights are sufficient to ensure that the radio propagation path, between each surface detector and its local tower, is predominantly lineof-sight (LOS). This guarantees predictable signal levels, thus allowing figures of merit for system availability to be calculated with confidence. However, for surface detectors located on the remote edges of a sector, the radio path length can approach $30 \mathrm{~km}$. At this range, the path will, in some cases, be near-LOS, rather than true-LOS, due to tropospheric anomalies. Consequently, a fade margin of $10 \mathrm{~dB}$ has been included in the design. This figure is typical for such links but has also been verified against the data from the measurement campaign: $10 \mathrm{~dB}$ will guarantee the required system performance level of $1 \times 10^{-6}$ uncorrected bit error rate, with a link availability of better than $99.8 \%$ of the time [30].

\subsection{Performance of the Observatory communication system}

The communication system for the Observatory has been running autonomously, and with very little user intervention, for just over a year. It has proved to be a reliable and robust system for data retrieval: to date over 1 billion data packets have been transmitted to the campus. Wireless LAN traffic activity for the 10-month period JanuaryOctober 2002 is illustrated in Fig. 18. This shows bi-directional activity based on a daily average. The solid, higher level, trace represents up-link traffic, i.e. from the surface detector of the EA, whilst the continuous line represents down-link

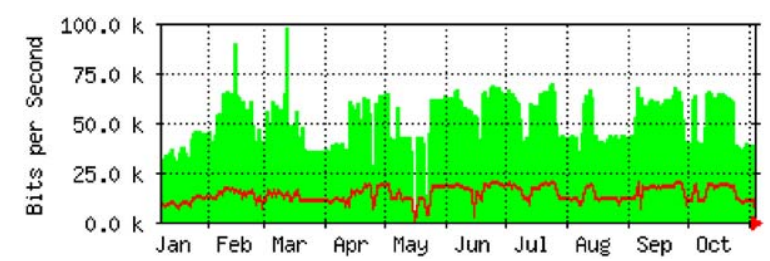

Fig. 18. Surface detector traffic activity over the period January-October 2002.

traffic. The two occasions of very low traffic during May 2002 are associated with unrelated maintenance and upgrade procedures of the Engineering Array.

A preliminary study of the bit error rates encountered in the EA system yield results at a level of around $1 \times 10^{-8}$ uncorrected bit errors. Thus, the system is operating well above the required level of performance of $1 \times 10^{-6}$ uncorrected bit errors and link availability should be very high in the full system.

The vast majority of the surface detectors are difficult to access so that high reliability of all electronic systems is a primary consideration. Final production versions of the radios where retrofitted in the EA during November 2002. To date these units have been operated for over 130000 unit-hours without failures, thereby validating both the design of the radios and the high reliability of the manufacturing and testing techniques used during production.

All radio systems require licensing before use: during November 2002, full licensing approval from the Argentinean National Commission for Communications (CNC) was granted for the radio transceiver employed in the EA wireless LAN system. This is an essential step prior to full deployment of the Observatory.

\section{Central Data Acquisition System}

\subsection{Overview}

The Central Data Acquisition System (CDAS) has been running with the EA since March 2001. The system was designed to assemble the triggers from the surface array detectors, to allow control 
of these detectors and to organise the storage of data. It is constructed using a combination of commercial hardware and custom-made, highlevel, software components. The system is designed to run continuously, with minimum intervention, and can be expanded for use with the full 1600 detector array, without major modifications. Data from the FD are recorded separately at the FD locations, although hybrid coincidences are identified within the SD data stream.

The primary role for the CDAS is to combine local trigger information, from both the SD stations and FD cameras, and to identify physical events, from the high level trigger (level 3 or T3). These triggers are used to generate requests for data relevant to the local triggers from all detector components. The CDAS is then used to combine and store these data in a timely manner to form a shower event. The CDAS also contains configuration and control mechanisms, the means to monitor system performance, and the tools to access and download SD monitoring, calibration, control and configuration data.

Except for triggering information, see Section 6.3, the CDAS and the FD data acquisition systems are completely independent. The marriage of FD and SD data is made off-line during the daytime following an FD run. Data are synchronised on the central storage hardware after each night of observation. The newly acquired data within the central storage is mirrored at the primary data mirrors located at the Lyon HEP Computer Centre (France) and at Buenos Aires (Argentina) every $4 \mathrm{~h}$, for later transfer to secondary mirrors sites such as that at the Fermi Laboratory. The data may then be transferred from a convenient mirror site to over 50 home laboratories.

The communication between applications within the CDAS is controlled using a central message routine manager called the Information Kernel. This manager allows formatted messages to be broadcast by producer applications (applications that need to advertise their status), and for consumer applications (applications that need to know about the status of others) to receive them on demand. All data, with one exception, are exchanged between the CDAS applications in human readable formatted ASCII and go through the Information Kernel manager. The exception is the large binary block of raw data coming from the SD stations. The data exchanged in raw format are data from local triggers, calibration blocks and FADC traces: these are the event data.

The CDAS runs on a network of six servers, each equipped with two $450 \mathrm{MHz}$ Intel processors and $256 \mathrm{MB}$ of memory. A RAID-5 disk storage system of $500 \mathrm{~GB}$ forms the central storage facility and an NTP GPS clock is used to synchronise the system times. We have adopted the LINUX Mandrake 8.2 distribution as the operating system. Only a very small fraction of the CPU power is used by the CDAS application on any of those servers. All software was developed in $\mathrm{C}$ or $\mathrm{C}++$.

\subsection{Data collection}

The data flow over the radio network, from individual SD stations to the central campus, is dealt with by a dedicated application called the Post Master. The Post Master is the end point of the communication backbone at the Observatory Campus (see Section 4), and is designed to dispatch information extracted from the different data streams of a local station to the other applications of the CDAS. As its name suggests, the Post Master application is used to read the data type contained in a radio frame and to forward it to the proper application within the CDAS so that specific data can be handled. When the data received from individual SD stations are split and sent in several radio frames, they are only reassembled and forwarded to clients by the Post Master after all the frames have been received.

The Post Master is used also to route data between the applications of the CDAS and the SD. Commands and configuration parameters can be transmitted, along with event requests, such as the level 3 trigger identified by the Central Trigger processor, and monitoring requests to the local stations. Software downloads over the communication link are also possible, thus enabling upgrades of the local DAQ software at the stations without the need to travel many kilometres to each one. 
Data received from each SD station belong to different data streams:

1: Local triggers, T2: a high priority stream, with time stamps and the type of trigger (threshold or time over threshold), is forwarded to the Central Trigger Application at a rate of $20 \mathrm{~Hz}$ per station.

2: Shower data and calibration data: Data in this stream are sent only when a request is received from the CDAS at an SD station. Shower events are split into small pieces and sent together with the T2 packets so that the available bandwidth is fully used. These data are forwarded to the 'event builder' application. Shower data are always accompanied by calibration data.

3: Control: This is a high priority stream that describes the state of the detector. It is forwarded to the central information kernel of the CDAS.

4: Calibration and monitoring information: This is a low priority data stream with the same pattern as the event stream. It is forwarded to the Monitoring Recorder application.

\subsection{Event triggering system}

The triggering system of the Observatory must fulfil two conditions. Firstly, it must be able to detect showers with high efficiency across the SD area when the initiating primary energy is above $10^{19} \mathrm{eV}$. Secondly, it must allow, and identify, cross-triggers (hybrid events) between the FD and SD systems. The first condition is straightforward to arrange: Monte Carlo studies made during the design phase of the project showed that use of a 5fold coincidence, with the detectors in a compact configuration, is expected to yield an efficiency greater than $95 \%$ for vertical showers at $10^{19} \mathrm{eV}$. This requirement also keeps the random coincidence rate at a few percent of the expected rate from physical events. Triggers from the FD are dealt with using separate algorithms but are forwarded to the SD system to construct the hybrid data set (see below).

The local DAQ system of each SD station is designed to generate low-level triggers. These T2 triggers are sent every second to the CDAS. The $\mathrm{T} 2$ requirements are such that the average rate is always less than $20 \mathrm{~Hz}$ so that at least $50 \%$ of the bandwidth is free for data transmission. This limitation does not reduce the global trigger efficiency (see below). At the CDAS, the T2s received from all stations are stored in a data block stamped according to the second to which they correspond. Once a block has existed for a time greater than the maximum transit time across the radio network (five seconds), it is transmitted to the Central Trigger processor and discarded.

For the EA, the Central Trigger processor is used to identify groups of stations that are clustered in time and space as SD events. These are the third level triggers, or T3s, and are created if they fulfil one of the following conditions:

1: The main trigger condition is based on both the time and the spatial clustering of the local triggers (T2) received from each station. First, time clusters are sought by centring a window of $\pm 25 \mu$ s on each T2. Clusters, with multiplicity of three or more, are then examined for spatial coincidences. For 3-fold coincidences, the triggered stations must lie within the first two hexagons centred on the station whose T2 served as centre of the time window. For a 4-fold coincidence, one station with a T2 may be as far away as in the 4th hexagon. This condition is, however, stronger than the 3 -fold coincidence requirement. Thus, all 4-fold triggers are also 3fold triggers. Once the spatial coincidence is verified, final timing criteria are imposed: each T2 must be within $(6+5 n) \mu$ s of the central one where $n$ represents the hexagon number. Fig. 19 illustrates the trigger conditions.

2: A random trigger is generated every $N$ min by selecting one of the T2s in an arbitrary manner, and promoting it to a T3. Currently, $N=30$ but values of 3 and 15 have also been used. The purpose of this trigger is to monitor randomly the FADC traces that satisfy the local trigger conditions and thus to verify the efficiency of the global trigger processor.

3: A 2-fold coincidence within $1 \mu$ s of the two neighbouring tanks (Carmen and Miranda: Section 3.2.4). These occur at $0.8 \mathrm{~Hz}$ and are scaled to $0.0017 \mathrm{~Hz}$ for transmission.

Once a trigger has been identified, a message requesting that all FADC trace information 


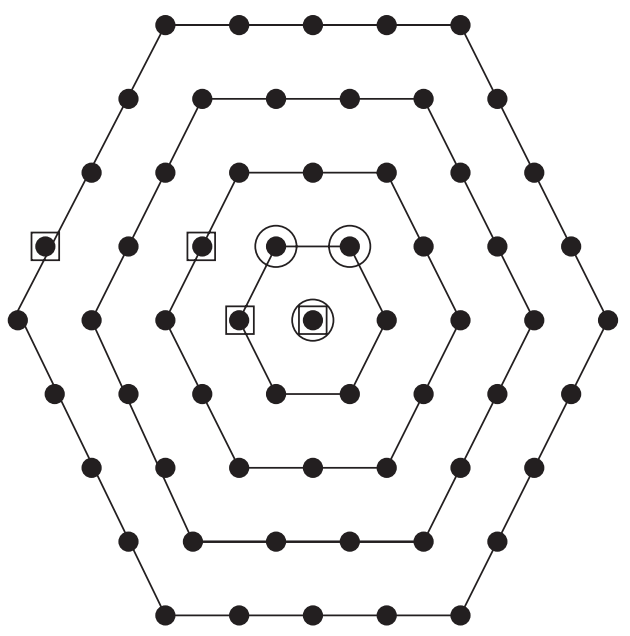

Fig. 19. Four hexagons, containing stations, are illustrated around a central surface station, for a portion of an idealised array. For a 3 -fold coincidence a T3 is issued if two T2 are found in the first two hexagons at an appropriate time with respect to the time of the T2 of the central station. For a 4-fold coincidence, the additional station may be as distant as in the 4th set of hexagons. Two examples of triggers are shown: a 4fold coincidence in which the triggered stations are identified by square, and a 3 -fold coincidence identified by circles. The subset of station closest to the centre in the 4-fold configuration also fulfils (as do all 4-fold configurations) the 3 -fold coincidence condition.

recorded within $30 \mu$ s of the central T2 is built by the Central Trigger processor and forwarded to the stations by the information kernel system and by the Post Master. Presently, this message is sent to all stations in the array, regardless of their distance from the central station that enters the trigger. The number of $\mathrm{T} 2 \mathrm{~s}$ for each station is recorded for monitoring purposes.

With the arrangements described, the total trigger rate during the first 8 months of 2002 was, on average, a little less than $0.01 \mathrm{~Hz}$ and about 200000 SD events were recorded.

The DAQ system of the fluorescence detector is completely independent of the CDAS. Local triggers are generated at each camera and those identified as T3 FD event triggers are logged by a local processor if a shower track can be found. After each night of operation, details of events recorded at the FD telescopes are transferred to the CDAS.
To build the hybrid event set, the high-level trigger (T3) information from the FD local DAQ system is transmitted on-line to the CDAS. This trigger information describes the geometry of the shower candidate. This includes the estimated time of arrival of the light front of the shower at the camera as well as the geometry of the Shower Detector Plane (SDP) (see Section 6.1.2). From this information, the time of impact of the shower at a ground position in the region of the $\mathrm{SD}$ stations is computed and a corresponding SD event T3 is constructed. All FADC traces recorded within $20 \mu \mathrm{s}$ of the computed time are assembled as a normal 'SD-only' event, but with the addition of the identification of the corresponding FD T3 trigger. Data from these triggers form the hybrid data set and are merged with the data collected by the FD DAQ and analysed offline. A hybrid event is therefore an 'FD-only' event together with a special SD event that contains all the surface station information that were, in space and time coincidence with the FD event.

Cross calibration of the SD and FD clocks was achieved by firing a laser into the sky and, at the same time, injecting a portion of the laser signal into one of the SD stations via an optical fibre. The time of laser pulse emission and the local time stamp recorded in the tank were then compared. The former was reconstructed from the laser track recorded in the telescopes and the latter from the local trigger generated by the light going through the tank.

\subsection{Monitoring}

The CDAS has been designed to provide the means to monitor both its own operation, as well as the slow control system at SD stations and various environmental parameters. The operation of the CDAS is monitored using a low level application that routinely checks that all software components are running correctly. This 'watch dog' system is used to re-initialise and re-launch any application that may have failed. In the period 1 January-31 August 2002, the CDAS was operational more than $90 \%$ of the time. Of the $10 \%$ time lost, about half was lost due to mains 
power failures and the rest due to system tests, upgrades and debugging.

The manager for the Information Kernel system, used to route messages, serves also as a monitoring tool at a higher level. Its architecture is based upon one central daemon, the message dispatcher, to which all messages are sent automatically and transparently. Any application wishing to distribute its status information, or wishing to know about the state of other applications in the system, connects to the Information Kernel and issues a monitoring request defining the class of message it wants to hear.

The 'message listener' applications range from monitoring applications to system-oriented ones, such as the message logger that is used to make all messages persistent. Applications have been developed that allow these messages to be browsed. Thus, the behaviour of the system can be monitored both on-line and off-line. The storage capacity is sufficient to keep the complete history of all messages exchanged in the system (around
$3 \mathrm{~Gb}$ per year of uncompressed ASCII files) for several years.

The SD stations monitoring information is collected upon requests from the CDAS at regular intervals (from every 5 to every $15 \mathrm{~min}$ ). This information is mainly concerned with the 'slow control' data, such as PMT and CPU board voltages and environmental parameters. Calibration data are also collected by the CDAS at a rate that can be varied: it has been set at 'every 10 min' for much of the EA operating period.

A graphical interface has been developed, using ROOT, an object oriented data analysis framework [31], to make and to present a preliminary analysis of the collected data. Examples of two monitoring plots are shown in Fig. 20.

An event display program (part of which can be seen in Figs. 23-25) has also been developed. This allows the selection, viewing and reconstruction of SD events that are stored on disk. This program, and the input/output and reconstruction libraries
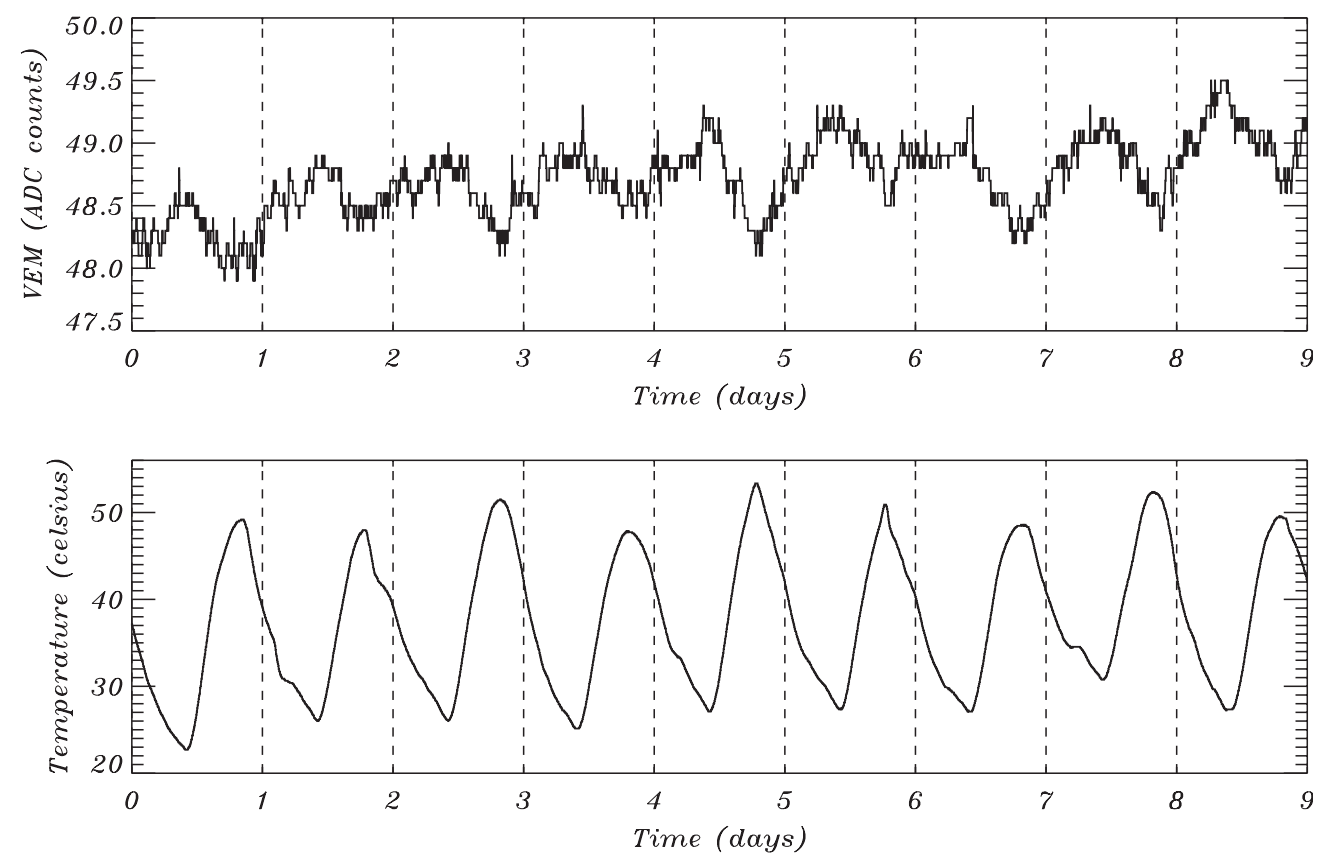

Fig. 20. Monitoring plot, as a function of time, of the VEM ADC value (top) and the board temperature (bottom) of PM2 in one of the SD tanks. Day/night variations, although at only the few per cent level, are clearly visible as is the correlation between the two parameters. 
that compose it, have also been used extensively for preliminary data analysis.

\section{Performance of the Engineering Array}

In this section, we demonstrate the performance of the Engineering Array by presenting data relating to some shower events. Where energies are mentioned in association with events, these should be regarded as indicative only.

\subsection{Events recorded with the Surface Detector}

Some $250000 \mathrm{~T} 3$ triggers have been recorded by the SD since data taking started in July 2001. In this section, the event selection procedures and preliminary analysis techniques will be explained and three particular events will be described.

\subsubsection{Event selection}

The different types of trigger of the surface detector were discussed in Section 6.3. Of interest here are those triggers in which at least a 3-fold coincidence has been recorded. As each detector operates in an autonomous manner, not all of the stations forming a T3 trigger will always have signals that are associated with a shower. Accordingly, a number of tests are being developed so that the number of spurious signals in an event is minimised: even an event of high multiplicity may contain one or more signals that are not associated with the shower.

The timing consistency within the tank pattern is a very efficient selection mechanism. If the times can be used to compute an arrival direction then it is likely that most of the signals will belong to a shower. However, one or more time-coincident signals may have occurred by chance. For example a single muon, especially if it makes a long track in a tank, or a low-energy shower arriving with its core close to one of the detectors, can readily satisfy the local T2 trigger requirement of a 3-fold coincidence between the PMTs at the 1.75 VEM level. In these cases, an effective rejection mechanism is to use the shape of the signal obtained from the FADC traces. By contrast to signals observed far from the core of high-energy events, those from muons or small local showers will have rather sharp pulses.

At this exploratory stage of event selection, a requirement has been set that, in 3 -fold events, two of the three stations of the event must have an 'area over peak' ratio of 1.2 or more. The 'area over peak' is defined as the integrated signal in VEM divided by the maximum of the signal in units of the peak of the VEM. This threshold means that pulses must have an integrated signal at least $20 \%$ larger than that from the response typically expected from a single muon. Additionally, for these events, the requirement that the area of the triangle formed by the three triggered stations be small has been explored: such events will be of lower energy than the sub-set in which dispersed stations are triggered. Finally, the time of triggering at each station must be compatible with the propagation of a plane shower front across the array at the speed of light. This last step is included as, initially, at the instant of the T3 trigger, the timing is known only with the accuracy of a few microseconds. Therefore, it is quite common to have three stations for which the times are incompatible with the propagation of a shower front at the speed of light. It is possible that threestation events will form a useful sub-set of data for the study of physics, such as anisotropy, close to the array threshold.

More extensive analysis is possible for events that have signals in four or more tanks. Again it is important to determine whether all of the station signals are part of the event, or whether some are random triggers: the techniques used with 3 -fold events remain applicable. In addition, a fit is used that describes the extreme front of the shower as a plane. If the residual to the fit is large, the fitting is repeated with stations removed until a reasonable fit is obtained or only two stations remain.

\subsubsection{Event reconstruction}

A very preliminary analysis is described that has been made as part of the evaluation of the technical performance of the EA. The events are given as examples rather than as a demonstration of the final analysis procedures that are currently under development. 
The first determination of the shower direction is made using a plane front. With this, one can reconstruct the zenith and azimuth angles to within about two degrees. To achieve better accuracy, a curved front is adopted, but this implies knowledge of both the impact point on the ground and of the shape of the extreme front of the shower. In the present reconstruction routine, a spherical form is used to describe the extreme front. Monte Carlo studies have been used to show that adopting a spherical front fit leads to a superior estimate of the incoming direction to that achieved with a planar fit. Other shapes for the extreme shower front are being explored.

The location of the core of the shower is determined, at this early stage, using a lateral distribution function (LDF) that describes the falloff of signal with distance by the approximation of a power law. The trial function used in the preliminary investigations is

$$
S(r(\mathrm{~km}))=S(1000) r^{-v} \text {, }
$$

for zenith angles $<60^{\circ}$,

where $v=4.80-1.26 \mathrm{sec} \theta$.

At larger zenith angles, an analysis that includes the effect of the geomagnetic field is required and a simple power law description of the fall of density with distance is inadequate.

Near to the core, the tank signal depends strongly on the depth of the first interaction of the incoming cosmic ray and therefore there are substantial fluctuations between the signals that would be recorded from a group of primary cosmic rays of the same mass and energy. Furthermore, observations as close as $100 \mathrm{~m}$ to the shower core are rare. Far from the core, statistical fluctuations in the signal size will dominate. It was shown during the design study, using guidance from earlier work, that fluctuations in the shower signal, in the energy range of interest to the Auger Observatory, have a broad minimum near $1000 \mathrm{~m}$ from the core. Accordingly, $S(1000)$, the signal at $1000 \mathrm{~m}$ from the shower axis, is used as the ground parameter from which an estimate of the primary energy can be made. The relationship between the energy in $\mathrm{EeV}\left(1 \mathrm{EeV} \equiv 10^{18} \mathrm{eV}\right)$ and $S(1000)$, measured in VEM, currently adopted is

$$
\begin{aligned}
& E(\mathrm{EeV})= \\
& 0.12\left(\sqrt{1+11.8(\sec \theta-1)^{2}} S(1000)\right)^{1.05} .
\end{aligned}
$$

Thus, in a vertical shower produced by a primary of $10 \mathrm{EeV}$, the signal at $1000 \mathrm{~m}$ from the core is 67.5 VEM.

The frequency of events as a function of the number of triggered stations and the distribution of shower cores are shown in Fig. 21 for a sample of events.

The declination of those events selected with shower energies $\approx 1.0-3.0 \mathrm{EeV}$ and $\theta<60^{\circ}$ of these events is shown in Fig. 22. It is seen that for these events the FWHM $\approx 75^{\circ}$ in declination. The declination distribution measured with an array of thin scintillators is much narrower. For example, the FWHM in declination at Volcano
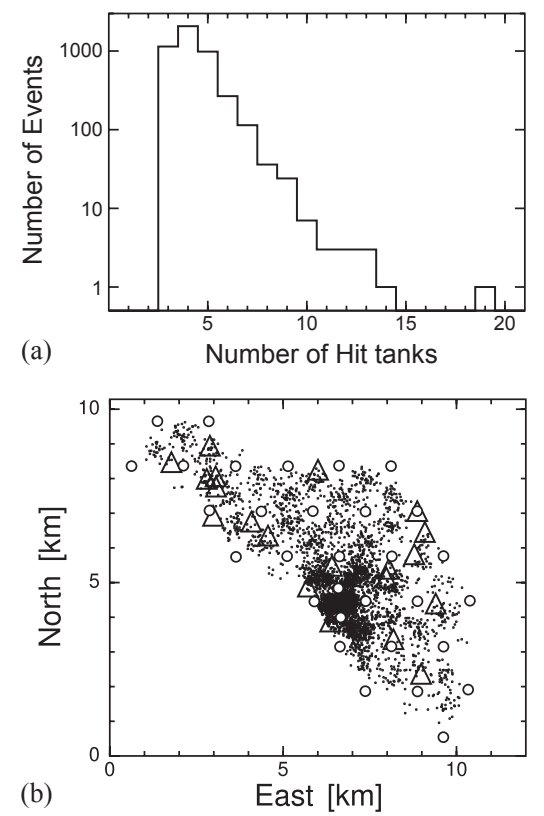

Fig. 21. (a) The multiplicity of the EA events (number of tanks triggered) selected for real physics events (see text). The few very high multiplicity events are very inclined events, which trigger a large number of tanks as the array is contracted in the shower plane. When the two adjacent detectors were triggered, only one hit was counted. (b) Reconstructed core locations. The circles represent the 32 active stations. Triangles indicate events above $10 \mathrm{EeV}$. Each dot represents a lower energy event, and an accumulation in the infilled portion of the array is evident. 
Ranch for events of all angles and in the same energy range was about $45^{\circ}[7,11]$. The Volcano Ranch array was at a similar altitude and latitude

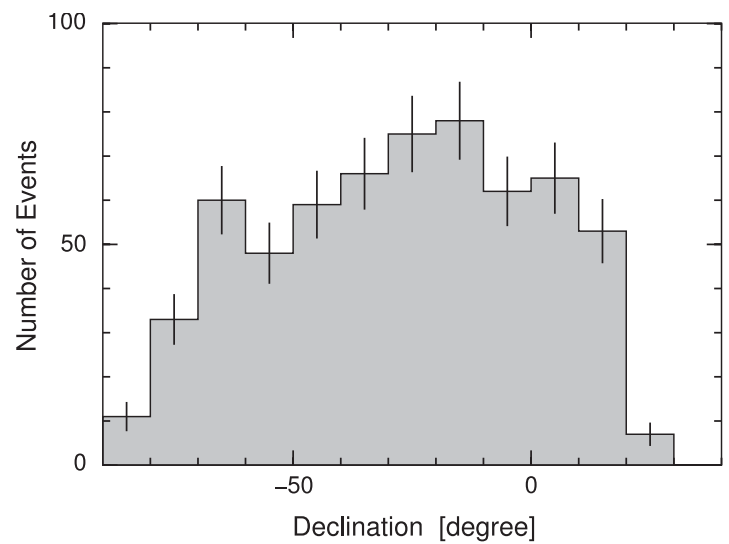

Fig. 22. The declination distribution of showers with $E \approx 1.0$ 3.0 EeV and $\theta<60^{\circ}$ recorded by the engineering array. The error bars shown in the $10^{\circ}$ bins are statistical. The FWHM $\approx 75^{\circ}$. (though in the northern hemisphere) to the Auger Observatory.

\subsubsection{Examples of single events}

7.1.3.1. Typical high-energy event. Event 255146 is a typical high-energy event of $\sim 5 \times 10^{18} \mathrm{eV}$ with a zenith angle of about $25^{\circ}$. The FADC traces from tanks near to the core of the shower show large, relatively short and structure-less signals, while those at greater distance show multiple peaks and signals with widths up to a few microseconds. Details of this event are shown in Fig. 23.

7.1.3.2. An event with some T2 triggers that are 'time-over-threshold'. The T2 trigger signals in the event shown in Fig. 23 were all of the simple 'threshold' type. A second type of trigger is also used that reflects the fact that some pulses are spread broadly in time and, although representing
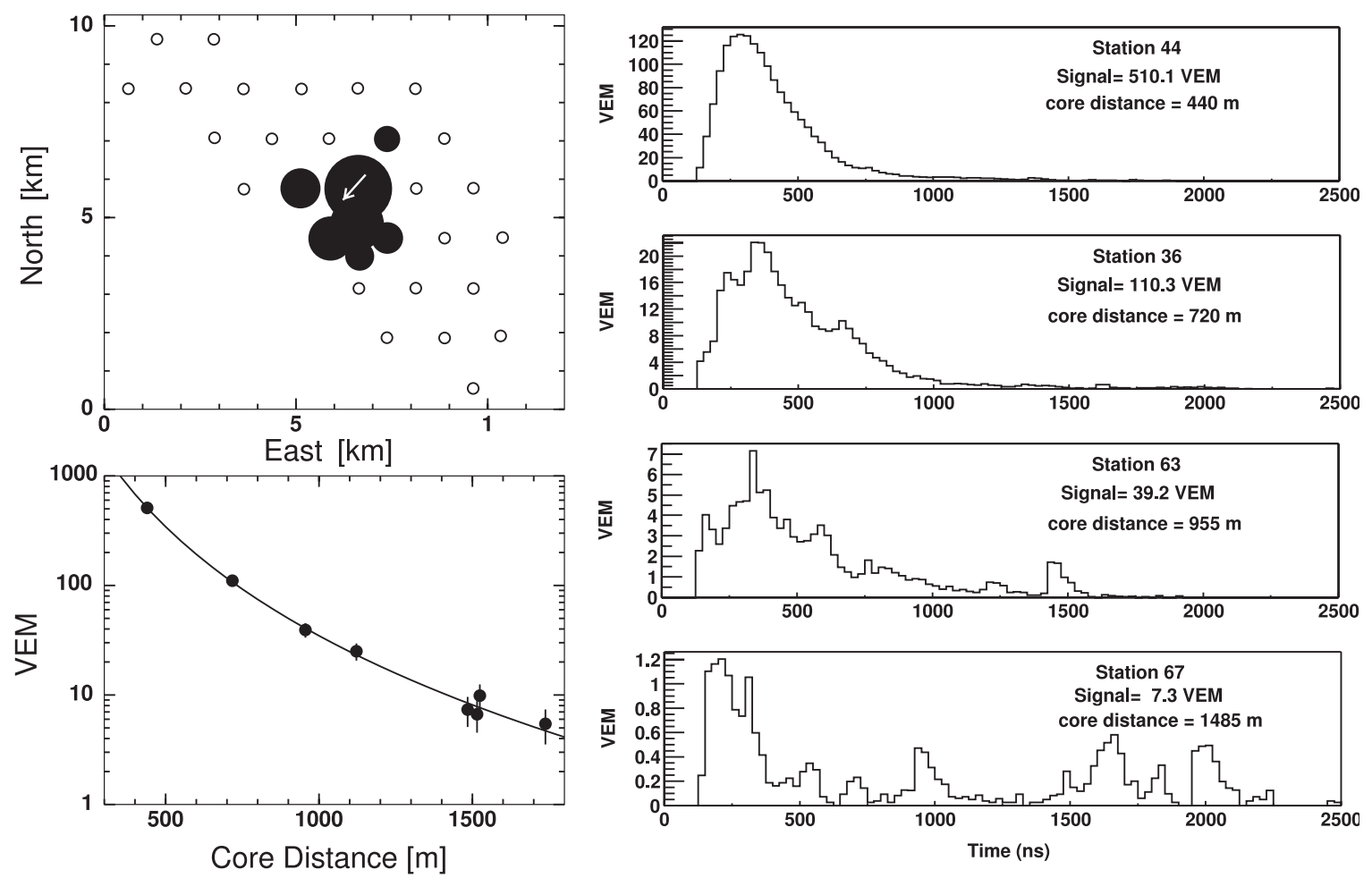

Fig. 23. Event 255146: a typical event of about $5 \times 10^{18} \mathrm{eV}$. Top left: The EA seen from above with the 8 triggered tanks. Lower left: The fit to the LDF appropriate for a shower of zenith angle $25^{\circ}$. Right: The FADC traces from four detectors at distances between 439 and $1485 \mathrm{~m}$ from the shower core. The signal sizes are in units of VEM. 

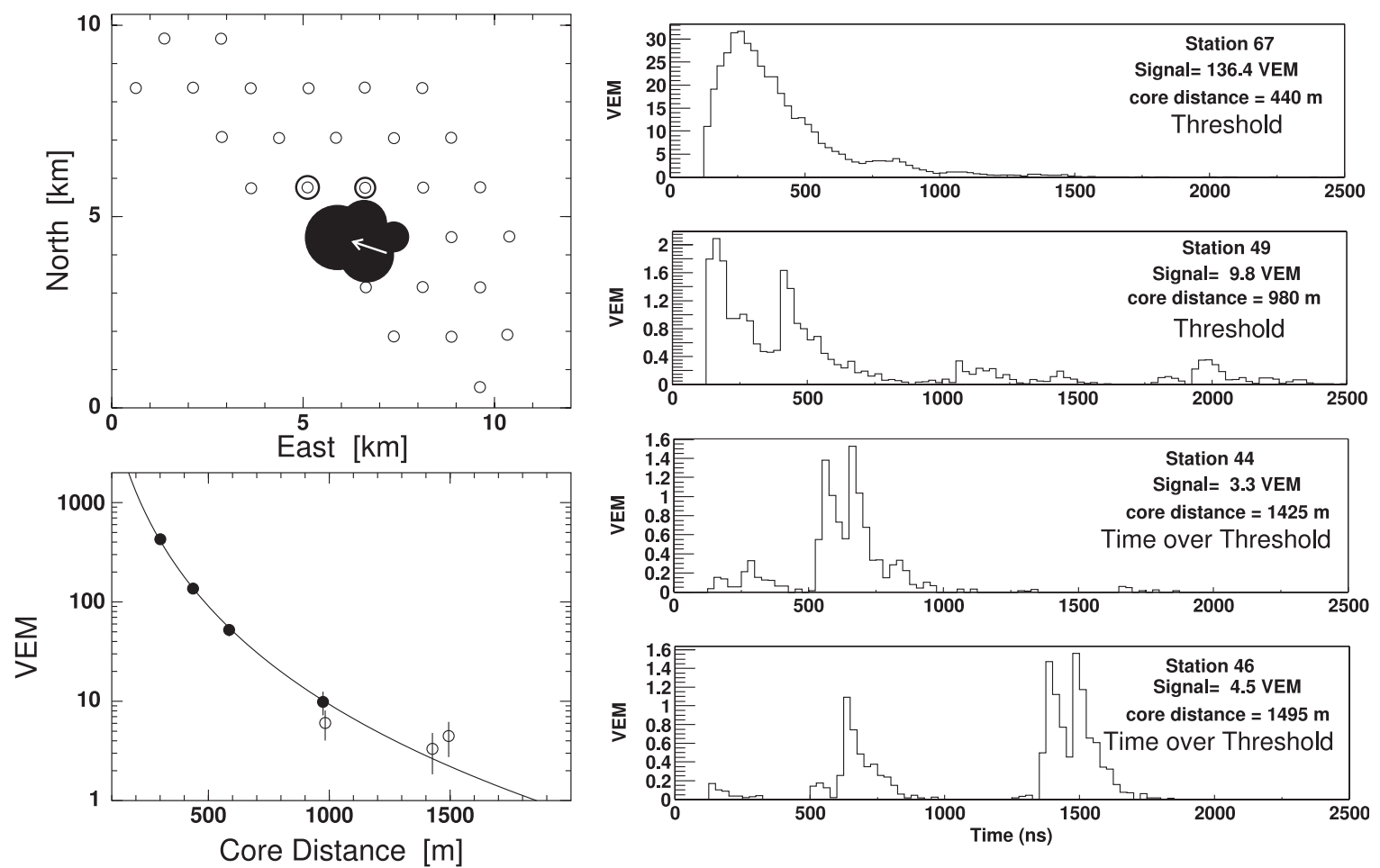

Fig. 24. Event 211377: the layout of the figure is as in Fig. 23. The FADC traces from stations 44 and 46 are for 'time-over-threshold' triggers.

a significant energy deposit, do not reach the simple trigger threshold. These are identified using the 'time-over-threshold' trigger described in Section 3.6. In Fig. 24 data are displayed from event 211377. Here two of the T2s were 'time-overthreshold' triggers. The same features of the event as in Fig. 23 are shown, including the FADC traces corresponding to the two time-over-threshold triggers.

\subsubsection{An event of high multiplicity and large} zenith angle. The highest multiplicity event recorded by the EA during its first year of operation is event 204272 for which details are shown in Fig. 25. In total, 20 tanks were triggered by a very inclined shower produced by a high-energy primary cosmic ray. The timing reconstruction gives a zenith angle of $81.4^{\circ}$ and a radius of curvature of $105 \mathrm{~km}$. The timing trigger at each station is within $50 \mathrm{~ns}$ of the reconstructed shower front, with a RMS of $22 \mathrm{~ns}$. The energy is $\sim 4 \times 10^{19} \mathrm{eV}$, if the shower core is constrained to lie within the boundary of the EA array (and larger if the core lies outside of the array). The large number of triggered detectors arises because of the high energy and the large zenith angle of the event. This kind of horizontal event, falling on the complete array, would have triggered about 40 detectors. The FADC responses from two detectors are shown and should be compared with the much broader signals seen in the events of Figs. 23 and 24 . These short time spreads are characteristic of what is seen in showers that arrive at large zenith angles from the vertical.

\subsection{Fluorescence detector events}

The FD telescopes of the Engineering Array were operated under stable conditions in the hybrid mode from December 2001 to March 2002. The average duty cycle of the detectors during this period was $10 \%$. Over a thousand 

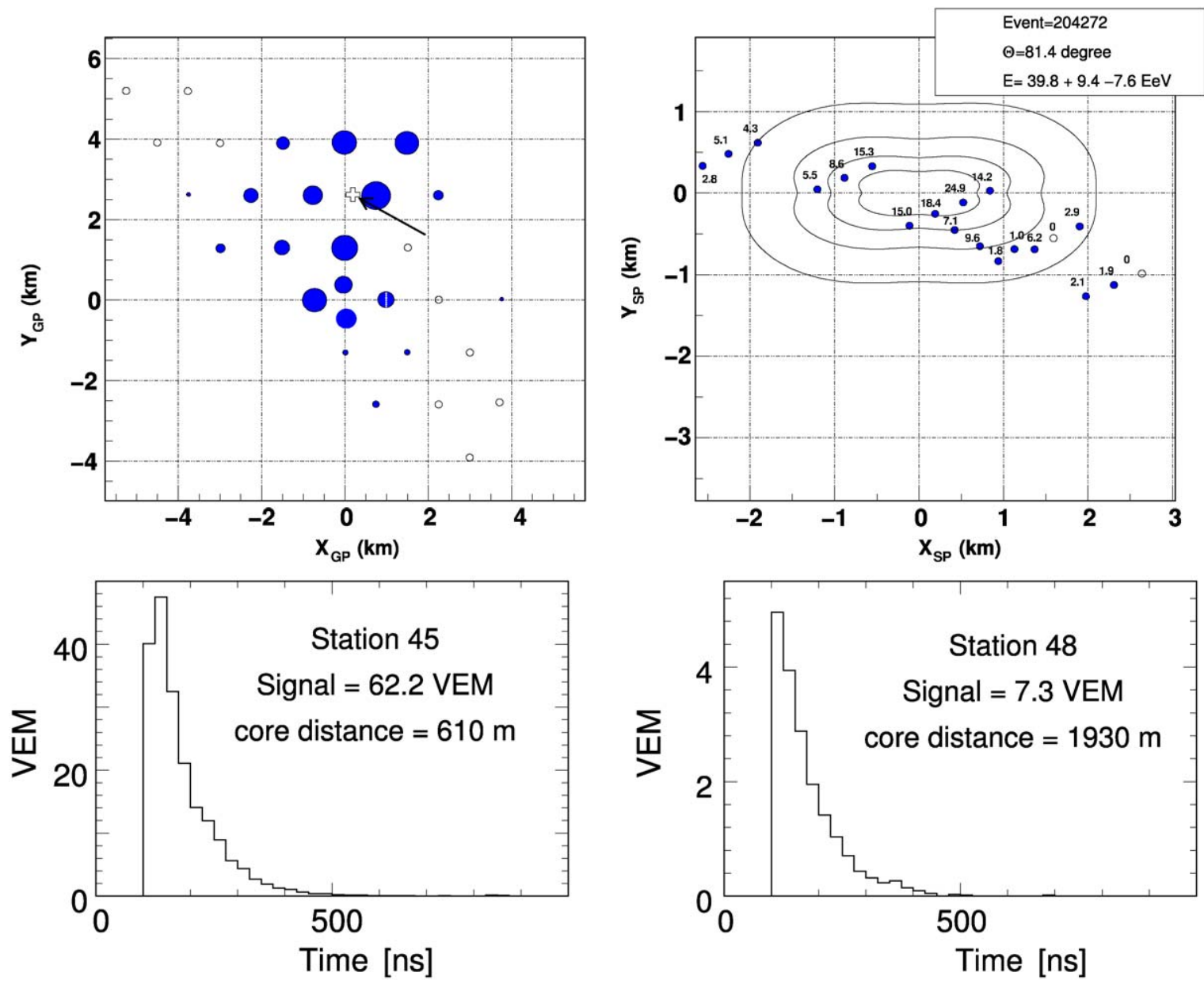

Fig. 25. Event 204272: the highest multiplicity event recorded during the first year of operation of the EA. Upper left. View of the EA with the 20 triggered tanks. The radius of each circle is proportional to the logarithm of the energy deposited in the detector. Empty circles indicate detectors that were not instrumented when this event was recorded. The arrow indicates the reconstructed direction and impact point. Upper right: the same event seen in projection in the shower plane. Density circles are at 100, 10, 4, 2 and 1 VEM. Lower figures: The FADC traces for two stations of the event. The compressed arrival time spread, as compared with what is evident in Figs. 23 and 24, are characteristic of inclined showers.

candidate cosmic ray showers were collected, including the hybrid events described in Section 7.3.

\subsubsection{Detector performance}

The stability of the detector was monitored during the run using the relative optical calibration system. The performance of the pixels was stable to within $2.5 \%$. A preliminary absolute calibration of the FD telescopes was performed. The drum illuminator and Rayleigh calibrations gave consistent results. A valuable cross-check of these calibrations comes from a piece-by-piece estimate of the calibration, based on the product of the measured efficiencies of the different telescope elements (filter, corrector ring, mirror, PMTs camera). On the basis of these results, a relative systematic uncertainty of $20 \%$ on the FD energy scale was estimated as arising from the calibration procedures. 


\subsubsection{Reconstruction of the shower axis}

Analysis procedures were developed to reconstruct the properties of the cosmic ray showers. The FD events were typically very clean, and the few random noise pixels included in events were rejected by applying simple topology-time constraints. The selected pixels were then used to determine the arrival direction of each shower.

As a first step, the SDP was reconstructed as follows. For each of the FD pixels, there corresponds a direction $\mathbf{r}_{i}$ in the sky. Ideally, the directions of the set of pixels that receive light from the shower defines the SDP. This plane contains the shower axis and intersects the FD in correspondence to these pixels. In practice, the best estimate of the normal vector to the SDP, $\mathbf{n}_{\mathrm{SDP}}$, was obtained by minimising the quantity:

$\chi^{2}=\Sigma_{i} w_{i}\left[\mathbf{n}_{\mathrm{SDP}} \cdot \mathbf{r}_{i}\right]^{2}$

where the signal measured in pixel $i$ was used as weight $w_{i}$. The SDP reconstruction procedure is robust and reliable. It was checked by reconstructing laser shots fired in the field towards known directions. Typically, errors in the $\mathbf{n}_{\text {SDP }}$ were found to be a few tenths of a degree.

The second step involves the reconstruction of the shower axis within the SDP, using the single FD telescope. For each shower pixel $i$, an average time $t_{i \text {,meas }}$ corresponding to the arrival of light from the shower in the pixel field of view can be measured from the FADC traces. The geometrical

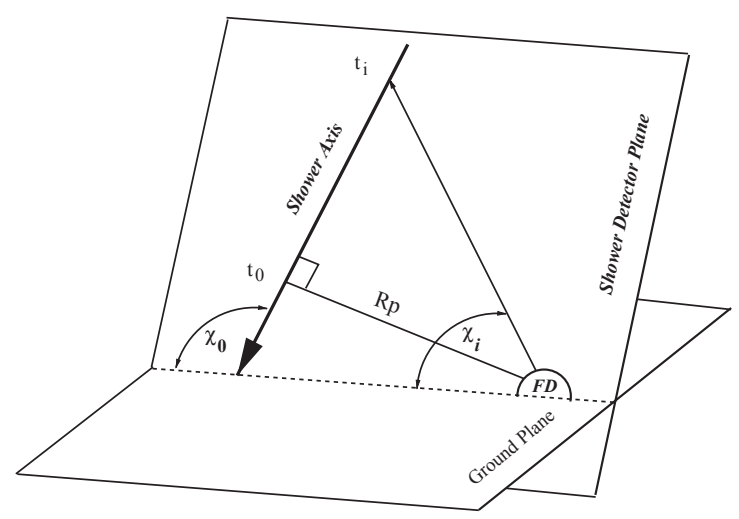

Fig. 26. SDP and shower geometry. parameters of the shower axis in the SDP (see Fig. 26) are related to the expected time $t_{i, \exp }$ of arrival of light at pixel $i$ by the following expression [32]:

$t_{i, \exp }=t_{0}+R_{p} / c \tan \left[\left(\chi_{0}-\chi_{i}\right) / 2\right]$

where $\chi_{i}$ is the direction of pixel $i$ projected onto the SDP, $\chi_{0}$ is the angle between the shower axis and the vector pointing from the detector to the shower landing point, $R_{p}$ is the shower distance of closest approach to the detector, $t_{0}$ is the time at which the shower front reaches the position of closest approach, and $c$ is the velocity of light.

The best fit to the three parameters $\chi_{0}, R_{p}$ and $t_{0}$ was obtained by minimising the $\operatorname{sum}\left(t_{i, \exp }-\right.$ $\left.t_{i \text {,meas }}\right)^{2}$. In this procedure, the precision of the fitted parameters depends on the geometry of the shower, and configurations in which there is significant curvature due to the large value of the argument of the tangent in Eq. (4) serve to constrain the shower geometry significantly. An example of a time fit for a shower candidate, landing at $19 \mathrm{~km}$ from the FD and having a zenith angle of $49^{\circ}$, is shown in Fig. 27.

\subsubsection{Longitudinal profile reconstruction}

One of the great advantages of the fluorescence technique is that it enables the longitudinal profile of an air shower to be measured, which in turn, by integration of the profile, yields the primary energy. A parameter with known sensitivity to mass composition, the depth of shower maximum, $X_{\max }$, can also be derived.

The procedures used for profile reconstruction were developed using showers and laser shots. The latter are particularly useful in this context as cross-checks of the detector calibration and of the assumptions made about the scattering of light in the atmosphere [27]. Here, the reconstruction of the shower profile of a particular event will be described in some detail (Fig. 28). The shower had a reconstructed zenith angle of $56^{\circ}$ with the core located $13 \mathrm{~km}$ in front of the fluorescence detectors.

The reconstruction procedure takes as input the calibrated ADC traces in all pixels, the 


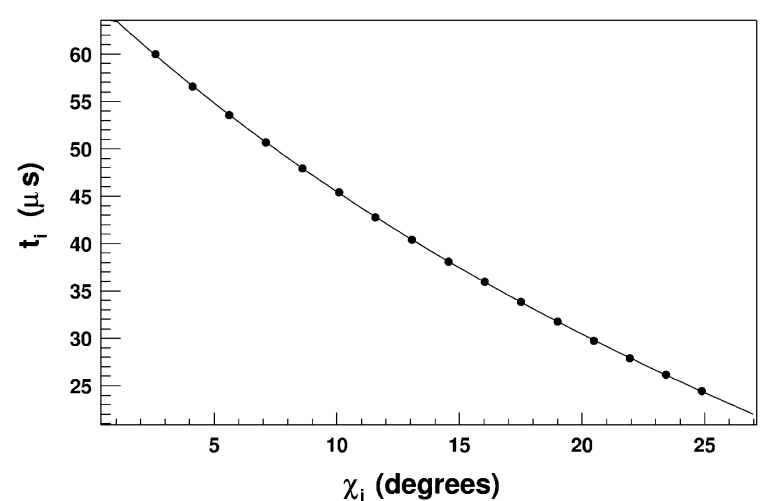

Fig. 27. An example of the geometry associated with shower reconstruction. The measured pixel time $t_{i}$ is plotted as a function of $\chi_{i}$, the direction of pixel $i$ projected onto the SDP. The closed dots are experimental data, the solid line is the result of the fit.

reconstructed geometry of the shower axis, as described in Section 7.2.2, and a model of aerosol scattering in the atmosphere. The first step is to determine the best estimate of the light arriving at the detector as a function of time. For each $100 \mathrm{~ns}$ time slot, the known geometry was used to calculate the position of the light spot on the camera. Then, signals in that time bin from all pixels whose centres are within an angle $\xi$ of the centre of the light spot were added together. The value of $\xi$ was chosen for each shower to maximise the signal to noise ratio averaged over the entire track: a typical value of $\xi$ is $1.3^{\circ}$. The signal is expressed in ADC counts. The endto-end procedures described in Section 4.1.7 allow these ADC numbers to be converted to the number of photons in each $100 \mathrm{~ns}$ time bin. The fluctuations in the light profile seen in Fig. 28 are in part due to photoelectron statistics and in part to slight non-uniformities in light collection at the camera.

Next, the geometry of the shower and the atmospheric scattering model were used to transform the light received at the detector to the light emitted from the shower axis as a function of slant depth (in $\mathrm{g} \mathrm{cm}^{-2}$ ). Parameters of the scattering model are currently the horizontal attenuation length due to aerosols, the scale height of the aerosols and a functional form for the aerosol

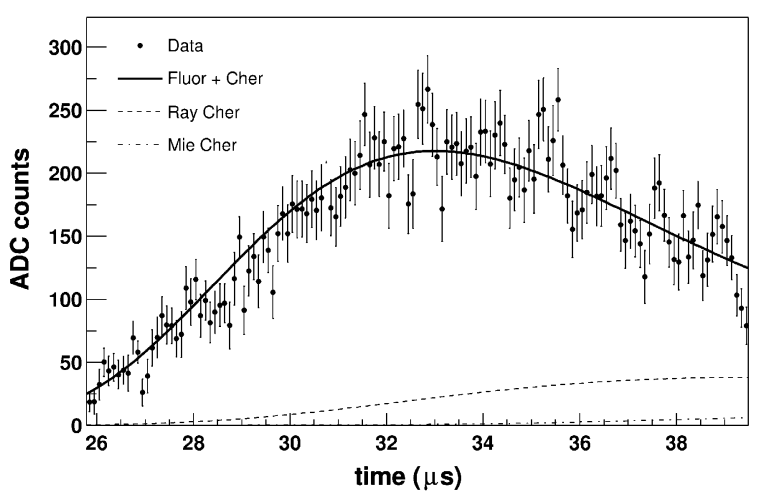

Fig. 28. The profile of received light as a function of time (closed dots). The direct Cherenkov light contribution to the profile is negligible in this event. Rayleigh and Mie scattered contributions are shown.

'phase function' (the normalised differential scattering cross-section) [27].

The light detected by the FD telescopes is a mixture of fluorescence light and direct and scattered Cherenkov light. The Cherenkov light contamination depends on the event geometry and the aerosol content of the atmosphere, and is generally small. The contamination cannot be accounted for without an estimate of the longitudinal profile (the goal of the analysis) of the shower. In one approach, an iterative procedure was used that starts by assuming that all of the light emitted at the track is fluorescence light. Then, assumptions about the fluorescence photon spectrum and yield [33] (with the yield having a small temperature and pressure dependence, and a small dependence on the age of the shower) were introduced to transform from the light emitted at the track to a first estimate of the longitudinal profile, the number of charged particles in the shower as a function of slant depth. This profile enables an estimate of the size of the Cherenkov beam, as it develops along the shower axis, to be made. It is then possible to calculate how much of this Cherenkov light is scattered and detected by the telescopes. This gives a new estimate of the pure fluorescence light at the detector. The procedure is repeated and, typically, convergence to a stable longitudinal shower profile was 


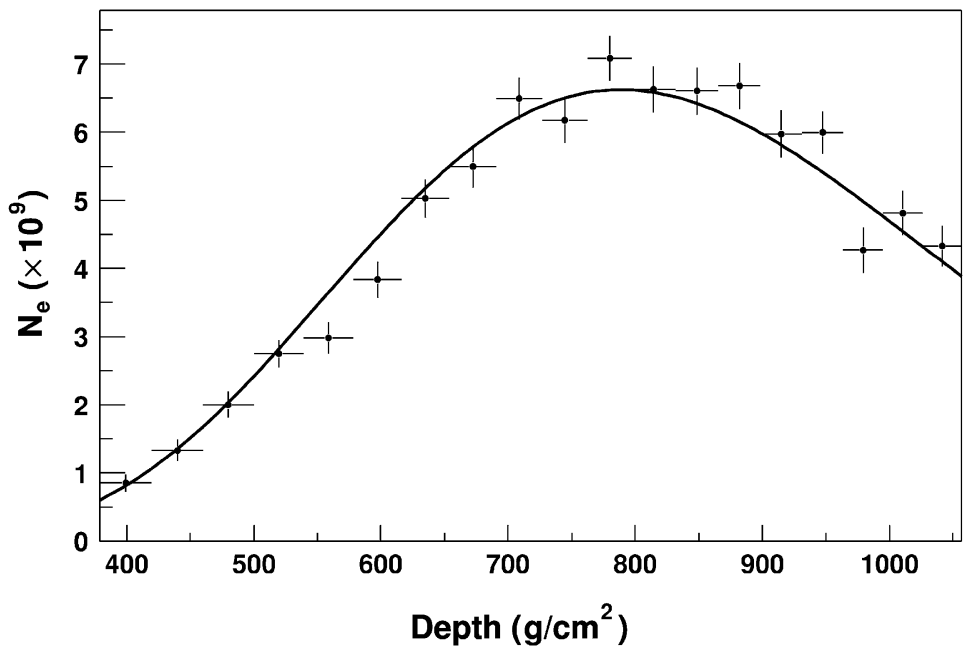

Fig. 29. The final longitudinal shower profile of the event of Fig. 28, with a fit to a Gaisser-Hillas function. Data have been rebinned for convenience of presentation. The geometry of the shower is such that the telescope views slant depths over a wide range, which includes the shower maximum. This preliminary analysis suggests a visible energy of $10^{19} \mathrm{eV}$.

obtained in less than four iterations. The fluorescence shower profile obtained after the iteration procedure is then fitted with a Gaisser-Hillas functional form.

In another approach, the Cherenkov contribution was included directly in the likelihood function of the shower profile fit. The Cherenkov contribution depends on the shower size, and is thus uniquely determined for any given trial shower profile. A small dependence on the shower model assumed could, in principle, be introduced by the functional form chosen for the shower profile (e.g. Gaisser-Hillas form), which however becomes negligible if the chosen form represents the experimental data well. Both methods of treating the Cherenkov contribution gave similar results. The best estimates of the three components of Cherenkov light for the example shower-the direct light and that scattered into the telescope field of view by Rayleigh and aerosol scattering - are also shown in Fig. 28.

In Fig. 29, the reconstructed longitudinal profile, together with a fit to a Gaisser-Hillas function, is shown. The fit yielded a value of $740 \mathrm{~g} \mathrm{~cm}^{-2}$ for $X_{\max }$. To estimate the visible primary energy, the Gaisser-Hillas curve was integrated and the resulting total track length multiplied by a mean energy loss rate of $2.19 \mathrm{MeV} / \mathrm{g} \mathrm{cm}^{-2}$ to give $1 \times 10^{19} \mathrm{eV}$. The energy estimates must be considered as preliminary, as detailed atmospheric monitoring was not available during the EA phase, and only average atmospheric model parameters have been used in the analysis chain.

\subsection{Hybrid events}

A crucial aspect of the Engineering Array evaluation is the observation and measurement of showers, simultaneously, by the fluorescence and surface detectors. The hybrid data set will be of paramount importance for establishing the validity of the absolute energy scale of the Auger Observatory and for determining the distribution of primary particle types (chemical composition). Data from the EA provide the first opportunity to demonstrate the reconstruction techniques that were explored previously only with Monte Carlo simulations [34]. Our methods are unique and differ from those that were used in the earlier HiRes/MIA data analyses [35] in which accurate clock synchronisation was 
lacking. Unlike a stereoscopic fluorescence detector, the hybrid feature of the observatory allows the exploitation of information about the time of the shower front arrival at one or more surface detectors to determine the geometry of the shower axis more precisely than with a monocular detector alone. Stereo FD measurements are not needed, although they will be useful for demonstrating our understanding of the atmosphere.

There are two parts to the hybrid geometric reconstruction. The first is the reconstruction of the SDP, which is done exactly as described in Section 6.1.2. The second task is to identify the shower axis within the SDP. The FD measurement of the angular velocity through its field of view does not determine the axis uniquely but does reduce the set of possible lines to only a 1-parameter family [32]. For any single water tank location, each of these possible axes gives a clear prediction as to when the shower front would arrive at that tank location. A time measurement, by only a single tank, therefore breaks the degeneracy and leads to a determination of the shower axis. Timing information from multiple tanks offers redundancy and the possibility of higher precision. Note that the geometric reconstruction of events recorded in the hybrid mode does not rely on measurements of the signal sizes at the tanks. Thus, in hybrid events, the SD signals are measured at core distances found independent of any SD reconstruction.

The EA was configured and operated to test the hybrid reconstruction methods but at energies well below $10^{19} \mathrm{eV}$ at which the Auger Observatory will be fully efficient. For hybrid events with only one or more tanks triggered in coincidence with an FD trigger, the median shower energy was close to $10^{18} \mathrm{eV}$. Over 70 hybrid events were reconstructed from data recorded during 4 months of operation.

Hybrid shower energies can be evaluated from the FD data using the techniques described in Section 7.2.3. The only difference here is that the more reliable hybrid geometric reconstruction method is used when converting the measured light flux $F(t)$ into the longitudinal shower development profile.
An example of the geometric reconstruction of a hybrid shower is shown in Fig. 30a. This shower was recorded in January 2002, before the timeover-threshold trigger was implemented (Section 3.6). The hybrid reconstruction is reliable even though only one water tank participated in the event. The upper plot in Fig. 30a indicates the fields of view of the prototype telescopes in bays 4 and 5 , each $30^{\circ} \times 30^{\circ}$. The track of pixels having fluorescence signals for this event lies in bay 4 , on the right. The curve indicates the fitted great circle, which determines the SDP. The lower left plot shows the timing fits. There are two curves showing the monocular and hybrid determinations of the function for $t$ described in Section 7.2.2 (Eq. (4)).

In the monocular case, the parameters $R_{\mathrm{p}}, \chi_{0}$, and $T_{0}$ are all determined from the plotted points. In the hybrid determination, the parameters are also constrained by the arrival time of the shower front at one tank. The lower right plot indicates the core location and arrival direction relative to the FD and the array of operating tanks. Both the monocular and hybrid reconstructions of the core location are shown. Monocular reconstruction works quite well when the curvature of the $t(\chi)$ function is accurately determined by the data. Hybrid reconstruction works well whether or not the curvature can be measured, and uses only timing information from the tank. The distance of the shower core to the tank is not assumed in the reconstruction. It has been demonstrated, using this method, that the core position found is always closest to the tank that has the greatest amplitude.

The longitudinal development deduced for this shower is shown in Fig. 30b. The left graph gives the light flux (photons $\mathrm{m}^{-2}$ per $100 \mathrm{~ns}$ ) at the detector as a function of time. The absolute FD calibration is used to convert the ADC value of each pixel to a light flux. For each 100 ns time bin, the resulting flux values are summed from those pixels that might have collected some of the signal, as determined using the reconstructed geometry and the optical spot size. The right graph in Fig. 30b shows the number of charged particles in the shower as a function of atmospheric slant depth, derived from the light flux and the known 
geometry. The integral of the fitted Gaisser-Hillas function gives a shower energy of approximately $3 \mathrm{EeV}$. The portions of signal attributable to direct and scattered Cherenkov light for this geometry are indicated in the left plot. The shower size profile is derived from the fluorescence light flux by subtracting the Cherenkov parts from the total flux. This event has a much greater Cherenkov

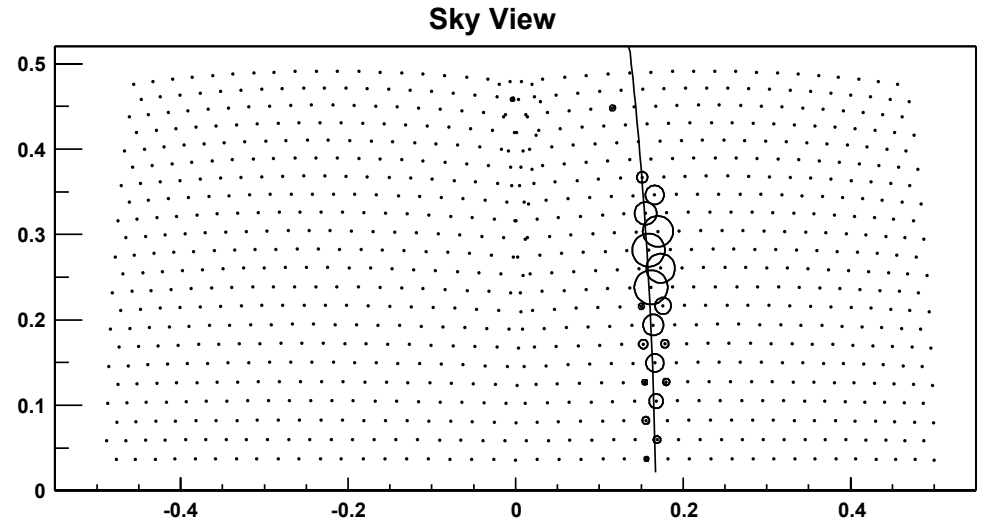

Pixel Time vs Angle
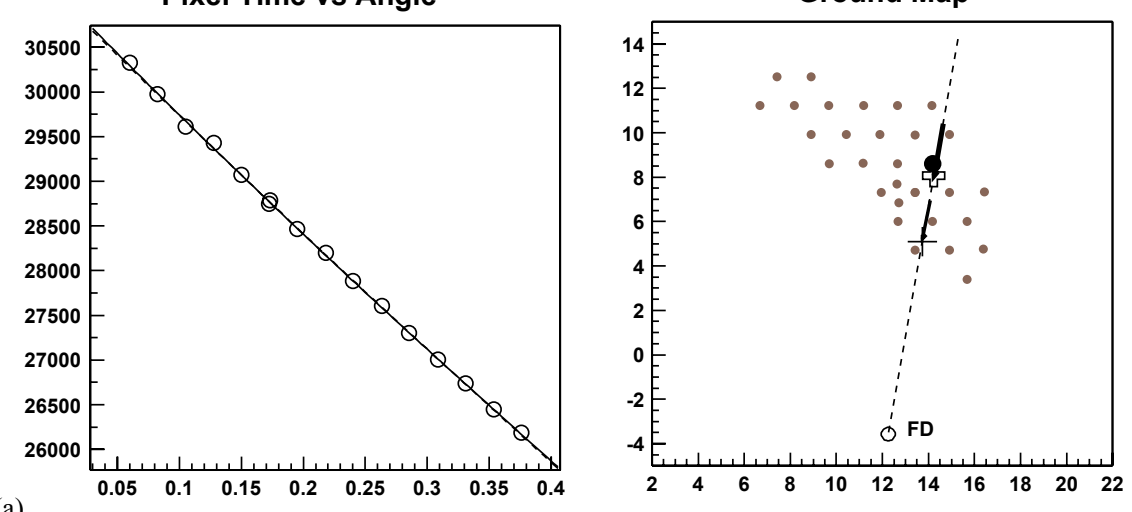

(a)
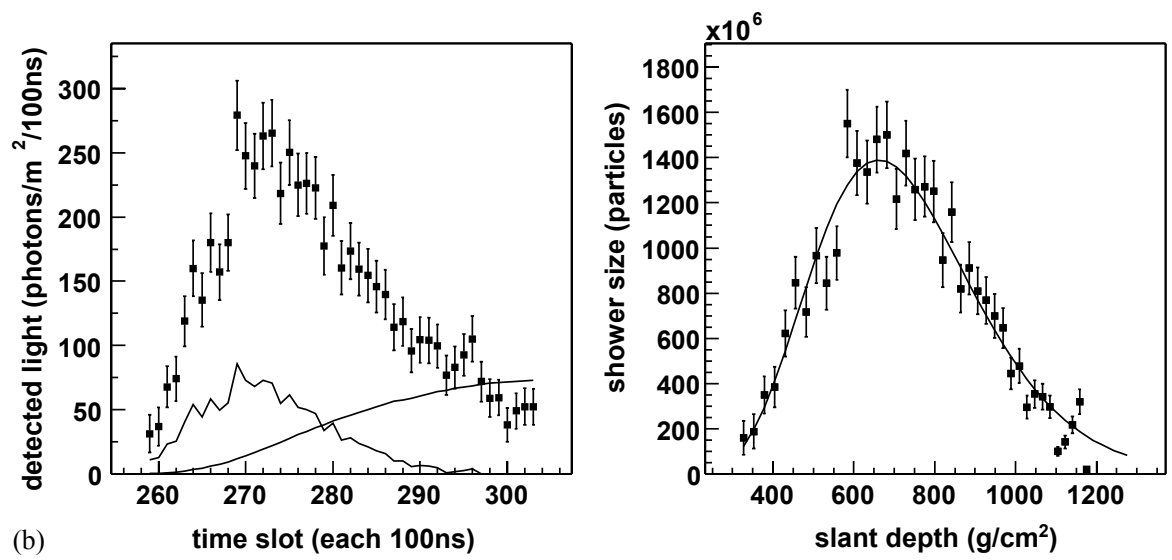
contribution than average, as the shower was moving in a direction towards Los Leones.

For hybrid events, the geometric and energy uncertainties depend on parameters such as the FD angular track length, the brightness of the light flux $F(t)$ and on the number of tanks recording the arrival times of the shower front. Full details of the analysis will be presented elsewhere. However, the present analysis has already shown that, for the larger EA showers, core locations can be found to a few tens of metres and the shower arrival direction to a small fraction of a degree. This bodes well for the full Auger Observatory at energies near $10^{19} \mathrm{eV}$ and above.

Most of the EA hybrid events are well below the energy needed for a T3 trigger. Therefore, with the EA, it has not been possible to compare FDdetermined energies directly with SD-determined energies on a shower-by-shower basis. It is nevertheless possible to examine the collection of hybrid events for FD/SD consistency or discrepancy. The core distance is known for each tank measurement, along with the FD energy determination for that shower. Using a mean lateral distribution function (derived either from Monte Carlo simulations or Haverah Park data), a prediction of the amplitude expected at each tank can be made. The measured amplitudes can be compared with those predictions with some statistical power. Many of the EA hybrid event tank measurements occur roughly $1 \mathrm{~km}$ from the core where shower development fluctuations are minimal. This test of hybrid consistency exercises all aspects of the hybrid reconstruction techniques and detector calibrations.

\section{Conclusions}

It has been demonstrated, through the successful operation of the engineering array of the Pierre Auger Observatory, that the design formulated in 1995 works extremely well. This has been shown through a description of the operation of various sub-components of the Observatory and by the detection of a wide range of events that satisfy a variety of trigger conditions. Of particular importance are the hybrid events in which measurements were made in the same showers with the fluorescence detector and with the surface detector. A pre-production array of a further 100 tanks and $2 \times 6$ telescope fluorescence detectors will become operational in late 2003 , with completion of the full instrument at the Southern site expected in 2005 .

\section{Acknowledgements}

We are very grateful to the following agencies and organisations for financial support: Gobierno de Mendoza, Comisión Nacional de Energía Atómica y Municipalidad de Malargüe, Argentina; the Australian Research Council; Fundacao de Amparo a Pesquisa do Estado de Sao Paulo, Conselho Nacional de Desenvolvimento Cientifico e Tecnologico and Fundacao de Amparo a

Fig. 30. (a) Geometric reconstruction of a hybrid air shower. The upper plot shows the fields of view for the two prototype FD telescopes and indicates the pixels in bay 4 whose signals contributed to the SDP determination. The circle sizes indicate the pixel charge integrals logarithmically. The lower-left plot shows the time when the spot passed the centre of each pixel vs. the angle associated with that pixel, $\chi$, with respect to the horizontal line within the SDP. (Time increases as this angle decreases.) Two curves of the expected functional form are drawn (monocular and hybrid reconstructions), but the curves may not be distinguishable for this event. The corresponding core locations are shown in the lower right plot: hybrid by the bold cross and black arrival direction, monocular by the thin cross and arrival direction. The azimuth of the arrival direction almost coincides with the line (dashed) joining the telescope location to the shower core. The position of the triggered tank is indicated by the large spot. The scale on both axes is in $\mathrm{km}$. (b) The longitudinal profile of the same event. On the left is a plot of the received light flux (photons $\mathrm{m}^{-2}$ per $100 \mathrm{~ns}$ ) as a function of time. Each point represents an independent 100 ns measurement. The right plot shows the shower size plotted against atmospheric slant depth. The smooth curve is a Gaisser-Hillas function fitted to the size points. In the left plot, the smoothly rising curve indicates the direct Cherenkov light, and the curve with the smaller amplitude is the flux due to light scattered from the forward Cherenkov beam. 
Pesquisa do Estado de Rio de Janeiro, Brasil; National Science Foundation of China, Ministry of Education of the Czech Republic (projects LA134 and LN00A006); Centre National de la Recherche Scientifique, Institut National de Physique Nucléaire et Physique des Particules (IN2P3/ CNRS), Institut National des Sciences de l'Univers (INSU/CNRS) et Conseil Régional Ile de France, France; German Ministry for Education and Research and Forschungszentrum Karlsruhe, Germany; Istituto Nazionale di Fisica Nucleare, Italy; Consejo Nacional de Ciencia y Tecnologia, Mexico; the Polish State Committee for Scientific Research (grant numbers PBZ KBN 054/P03/ 2001, 2 PO3B 11024 and 2 PO3D 011 24); Ministry of Education, Science and Sport of the Republic of Slovenia; MCyT (grant FPA 20013837) and Xunta de Galicia (grant PGIDIT02 PXIC 20611PN), Spain; Particle Physics and Astronomy Research Council, UK, the University of Chicago, the US Department of Energy the US National Science Foundation, USA, and UNESCO.

\section{References}

The references to notes written by the Auger Collaboration (GAP notes) are accessible via www.auger.org/admin/GAP_NOTES

[1] K. Greisen, Phys. Rev. Lett. 16 (1966) 748.

[2] G.T. Zatsepin, V.A. Kuzmin, Sov. Phys. JETP Lett. 4 (1966) 78.

[3] M. Takeda, et al., Astropart. Phys. 19 (2003) 135.

[4] T. Abu-Zayyad, et al., Phys. Rev. Lett., astro-ph/0208243, in press;

T. Abu-Zayyad, et al., Astropart. Phys., astro-ph/0208301, submitted.

[5] M. Nagano, A.A. Watson, Rev. Mod. Phys. 27 (2000) 689.

[6] X. Bertou, et al., Astropart. Phys. 14 (2000) 121.

[7] M.A. Lawrence, R.J.O. Reid, A.A. Watson, J. Phys. G 17 (1991) 733 and in: Catalogue of Highest Energy Cosmic Rays, World Data Center C2 for Cosmic Rays. Institute for Physical and Chemical Research, Tokyo, No. 1, 1980, pp. 61-97, 98-99.

[8] R.M. Baltrusaitis, et al., Nucl. Instr. and Meth. A 240 (1985) 410.

[9] Auger Collaboration Presentations, Proceedings of the 27th ICRC, Hamburg, 2001, pp. 699-787;
Pierre Auger Project Design Report, The Auger Collaboration, 2nd Edition, Fermi Laboratory, November, 1996, Revised March 14, 1997.

[10] I. Allekotte, et al., J. Phys. G: Nucl. Part. Phys. 28 (2002) 1499.

[11] J. Linsley, Phys. Rev. Lett. 10 (1963) 146, in: Catalogue of Highest Energy Cosmic Rays, World Data Center C2 for Cosmic Rays, Institute for Physical and Chemical Research, Tokyo, No. 1, 1980, pp. 5-59, 98-99.

[12] C.L. Pryke, J. Lloyd-Evans, Nucl. Instr. and Meth. A 354 (1995) 560;

C.L. Pryke, Nucl. Instr. and Meth. A 394 (1997) 420.

[13] A.K. Tripathi, Nucl. Instr. and Meth. A 504 (2003) 1.

[14] A. Cordero, et al., Proposal for the Optical System of the Fluorescence Detector of the Auger Project, Auger technical note, GAP-96-039.

[15] R. Sato, et al., Corrector Plate with an Annulus Shape, Auger technical note, GAP-99-014.

[16] G. Borreani, et al., IEEE Trans. Nucl. Sci. NS-48 (2001) 406.

[17] G. Borreani, et al., IEEE Trans. Nucl. Sci. NS-48 (2001) 395.

[18] G. Matthiae, et al., Proceedings of the 27th ICRC, Hamburg, 2001, p. 733;

E. Bollmann, M. Vlcek, Quality measurements of the mirror segments for the second prototype fluorescence telescope, Auger note: GAP-2001-017, 2001.

[19] M. Ambrosio, et al., IEEE Trans. Nucl. Sci. NS-48 (2001) 400.

[20] M. Ambrosio, et al., The Photomultipliers of the Auger Fluorescence detector, Proceedings of Third International Conference on New Developments in Photodetection, Nucl. Instr. and Meth. A 504 (2003) 234;

P. Facal San Luis et al., The test system for the photomultipliers of the Fluorescence Detector, Auger note: GAP-2001-042, 2001.

[21] S. Argiro, et al., Nucl. Instr. and Meth. A 461 (2001) 440;

S. Argiro, et al., IEEE Trans. Nucl. Sci. NS-48 (2001) 444.

[22] H. Gemmeke et al., Proceedings of the 27th ICRC, Hamburg, 2001, p. 699, 737.

[23] M. Kleifges, et al., IEEE Trans. Nucl. Sci. NS-50 (4) (2003).

[24] J.T. Brack, et al., Astropart. Phys. 20 (2004) 653.

[25] B. Dawson, et al., An absolute calibration of the bay 4 telescope using remote laser shots on October 20, 2001, Auger note: GAP-2002-010, 2002.

[26] J.A.J. Matthews, for the Pierre Auger Collaboration, SPIE Proceedings, Vol. 4858, (2003) 121.

[27] J.A.J. Matthews, et al., Proceedings of the 27th ICRC, Hamburg 2 (2001) 745.

[28] B. Keilhauer, et al., Proceedings of the 28th ICRC, Tsukuba, 2003, p. 879.

[29] Rappaport, T.S., Wireless Communications, Principles and Practice, Prentice-Hall, Englewood Cliffs, NJ, pp. 238-254, ISBN 0-7803-1167-1 (Chapter 5.7). 
[30] The Pierre Auger Project: Technical Design Review, Section 3.4: Surface Detector Wireless LAN Communications Network.

[31] R. Brun, F. Rademakers, Nucl. Instr. and Meth. A 389 (1997) 81 http://root.cern.ch.
[32] P. Sommers, Astropart. Phys. 3 (1995) 349.

[33] F. Kakimoto, et al., Nucl. Instr. and Meth. A 372 (1996) 527.

[34] B. Dawson, et al., Astropart. Phys. 5 (1996) 239.

[35] T. Abu-Zayyad, et al., ApJ 557 (2001) 686. 